UNIVERSITY OF SZEGED, FACULTY OF MEDICINE DEPARTMENT OF DERMATOLOGY AND ALLERGOLOGY DOCTORAL SCHOOL OF CLINICAL MEDICINE

\title{
IDENTIFICATION OF NEGATIVE REGULATORS OF THE CUTIBACTERIUM ACNES-INDUCED INNATE IMMUNE ACTIVATION IN HUMAN EPIDERMAL KERATINOCYTES
}

Ph.D. thesis

Lilla Erdei

Supervisor:

Kornélia Ágnes Szabó, Ph.D.

Szeged

2020. 


\section{Publications related to the subject of the thesis}

I. Lilla Erdei, Beáta Szilvia Bolla, Renáta Bozó, Gábor Tax, Edit Urbán, Lajos Kemény, Kornélia Szabó. TNIP1 regulates Cutibacterium acnes-induced innate immune functions in epidermal keratinocytes. Front Immunol. 2018 Sep 24;9:2155. doi: 10.3389/fimmu.2018.02155.

IF: 4,716 (Q1) (Independent citation: 1 Self citation: 0 Cumulative: 1)

II. K. Szabó, L. Erdei, B. Sz. Bolla, G. Tax, T. Bíró, L. Kemény. Factors shaping the composition of the cutaneous microbiota. Br J Dermatol. 2017 Feb;176(2):344-351

IF: 6,129 (D1) (Independent citation: 12 Self citation: 4 Cumulative: 16)

III. Lilla Erdei, Beáta Szilvia Bolla, Renáta Bozó, Gábor Tax, Edit Urbán, Katalin Burián, Lajos Kemény, Kornélia Szabó. TNFAIP3 negatively regulates Cutibacterium acnesinduced innate immune events in epidermal keratinocytes. (Accepted for publication. "Published in Acta Dermato-Venereologica by Society for Publication of Acta Dermato-Venereologica" https://www.medicaljournals.se/acta/content/abstract/10.2340/00015555-3707)

\section{Other publications}

IV. Szabó, K; Bolla, BSz; Erdei, L; Kemény, L. A bőrünkön élő mikrobák szerepe az egészséges bőrben és az acne vulgaris kialakulása során. ORVOSTOVÁBBKÉPZÖ SZEMLE 24 : 12 pp. 26-30. , 5 p. (2017)

V. Megyeri K, Orosz L, Bolla S, Erdei L, Rázga Z, Seprényi G, Urbán E, Szabó K, Kemény L. Propionibacterium acnes induces autophagy in keratinocytes: involvement of multiple mechanisms. J Invest Dermatol. 138(4) 750-759. (2018)

IF: 6,290 (D1) (Independent citation: 1 Self citation: 1 Cumulative: 2)

VI. Beáta Szilvia Bolla, Lilla Erdei, Edit Urbán, Katalin Burián, Lajos Kemény and Kornélia Szabó. Cutibacterium acnes bacterium affects the properties of the skin barrier. (Submitted for publication) 


\section{Table of Contents}

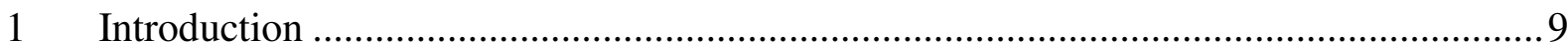

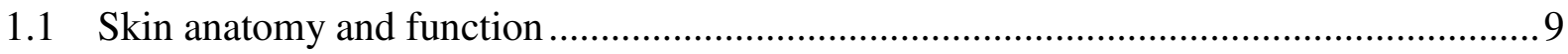

1.2 Localization and composition of the cutaneous microbiota ......................................... 10

1.3 The role of cutaneous microbiota in healthy skin and the pathogenesis of different skin

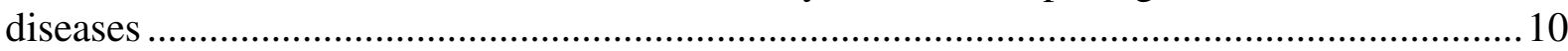

1.4 Skin microbiota-induced innate immune activation in keratinocytes ............................11

1.4.1 C. acnes recognition by human epidermal keratinocytes and their role in acne

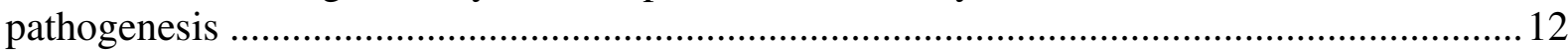

1.5 Factors contributing to the negative regulation of TLR signaling pathways ................. 14

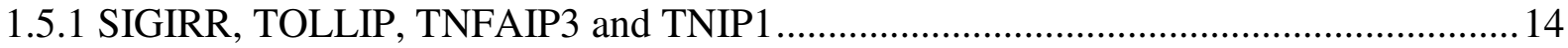

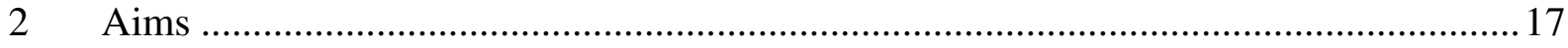

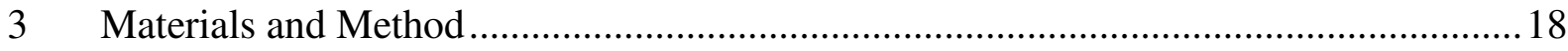

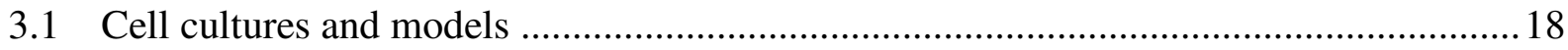

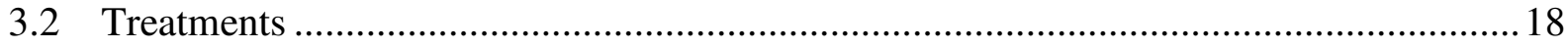

3.3 Transfection, plasmids and siRNA-mediated gene silencing .................................... 19

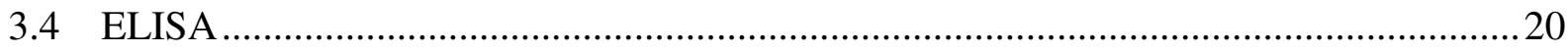

3.5 RNA isolation, cDNA synthesis and real-time RT-PCR .........................................20

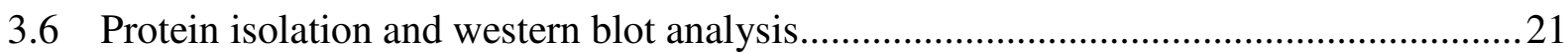

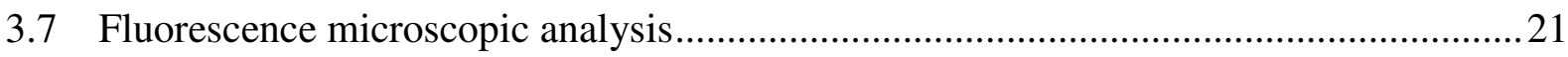

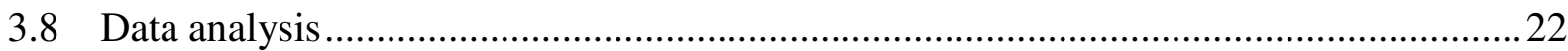

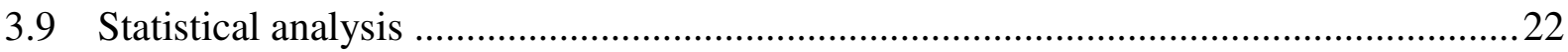

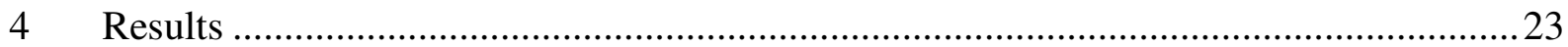

4.1 Studying the expression levels and the possible role of SIGIRR in the C. acnes-induced

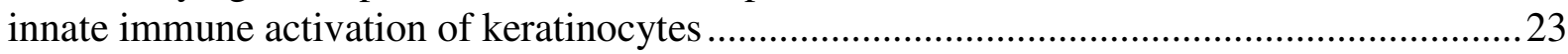


4.2 Studying the expression levels and the possible roles of TOLLIP, in the C. acnes-

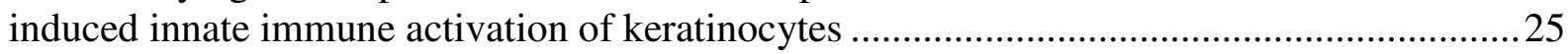

4.3 Studying the expression levels and the possible role of TNFAIP3 in the C. acnes-

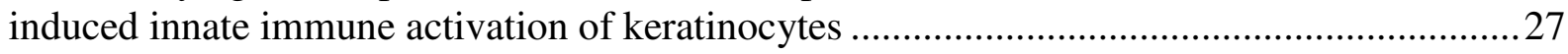

4.3.1 C. acnes bacterium increases TNFAIP3 expression in keratinocytes ...........................28

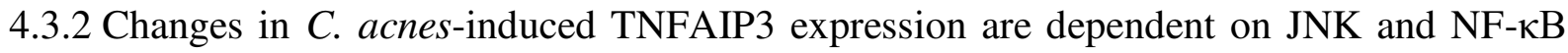

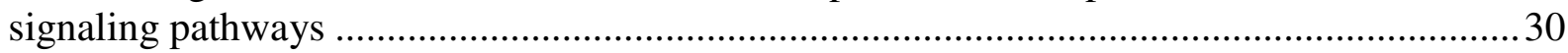

4.3.3 TNFAIP3 affects the basal and C. acnes-induced levels of inflammatory mediators in

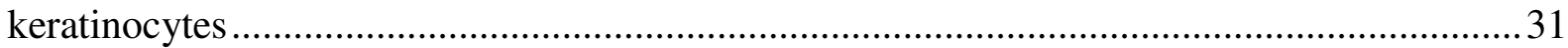

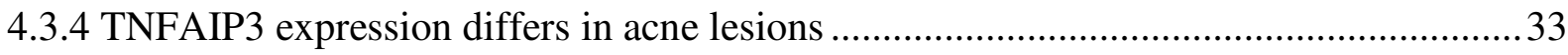

4.4 TNIP1 is expressed in keratinocytes and its expression increases in the presence of

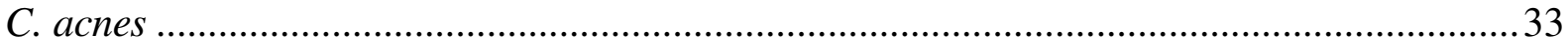

4.4.1 C. acnes-induced expression changes of TNIP1 are regulated by different signaling

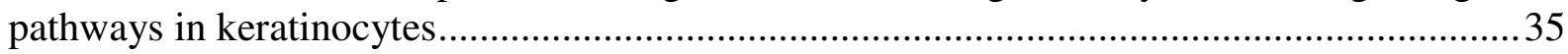

4.4.2 Regulation of TNIP1 levels in response to C. acnes bacterium is not specific for HPV-

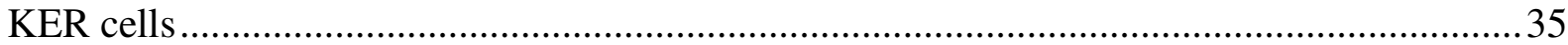

4.4.3 TNIP1 down-regulates both constitutive and C. acnes-induced inflammatory cytokines

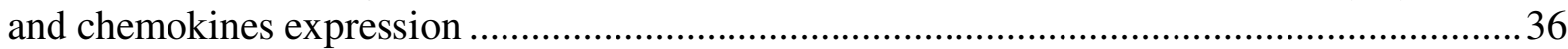

4.4.4 ATRA induces TNIP1 expression and affects the levels of downstream targets of the

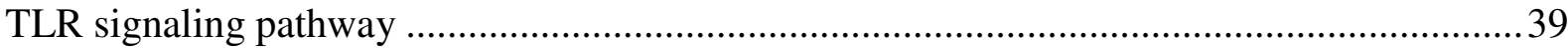

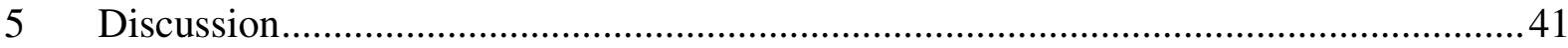

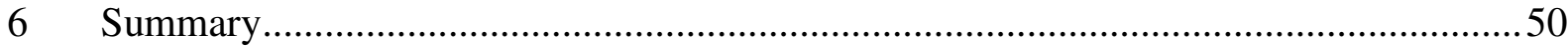

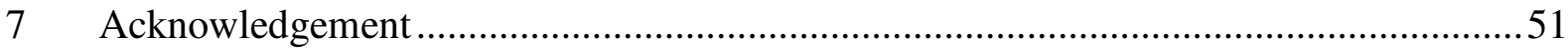

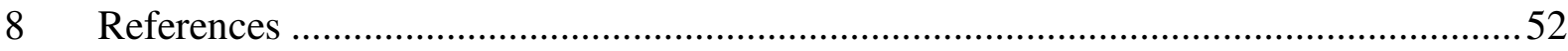




\section{Abbreviation}

AB/AM
AD
AMP
AP-1
ATRA
C. acnes
C/EBPB
CCL5
ChIP
CREB
DAPI
DMSO
DRE
DREAM complex

antibiotic/antimycotic

atopic dermatitis

antimicrobial peptide

activator protein 1

all-trans retinoic acid

Cutibacterium acnes

CCAAT enhancer binding protein beta

$\mathrm{C}-\mathrm{C}$ motif chemokine ligand 5

chromatin immunoprecipitation

cAMP response element-binding protein

4', 6-diamidino-2-phenylindole

dimethyl sulfoxide

downstream-responsive element

consisting of E2F dimerization partner (DP), retinoblastoma (RB), E2F

proteins, and the multivulva class B (MuvB) complex

ELISA

enzyme linked immunosorbent assay

ERK1/2

extracellular signal-regulated kinases 1 and 2

FADD

Fas associated via death domain

GSK3

Glycogen synthase kinase 3

hBDs

HLMVEC

human $\beta$ defensins

human lung microvessel endothelial cells

HPV-KER

Human Papillomavirus immortalized keratinocyte cell line

IKKi

IKKa

IKK $\beta$

IL

IL1RL1

IRAK

JNK

LPS

MAPKs

MOI

MYD88

NEMO

NF-кB

inhibitor of nuclear factor kappa B kinase subunit epsilon

inhibitor of nuclear factor kappa B kinase subunit alpha

inhibitor of nuclear factor kappa B kinase subunit beta

interleukin

interleukin 1 receptor like 1

interleukin 1 receptor associated kinase

c-Jun N-terminal kinase

lipopolysaccharides

mitogen-activated protein kinases

multiplicity of infection

MYD88 innate immune signal transduction adaptor

inhibitor of nuclear factor kappa B kinase regulatory subunit gamma (IKBKG)

nuclear factor kappa-light-chain-enhancer of activated B cells

normal human epidermal keratinocyte

ex vivo organotypic skin 
p105 nuclear factor kappa B subunit 1 and 2
p38

PAMPs and DAMPs pattern- and damage-associated molecular patterns

PBS

PSO

phosphate-buffered saline

RARE

psoriasis

RARs

retinoic acid response element

RIP1

retinoic acid receptors

S. epidermidis

S. aureus

SCFA

SD

SE

SIGIRR

SNP

SOCS1

SP1

receptor interacting protein 1

Staphylococcus epidermidis

Staphylococcus aureus

short chain fatty acid

standard deviation

standard error

single Ig and TIR domain containing

single nucleotide polymorphisms

suppressor of cytokine signaling 1

Sp1 transcription factor

SP3

STAT1

STAT3

Sp3 transcription factor

signal transducer and activator of transcription 1

signal transducer and activator of transcription 3

TAK1

TBK1

mitogen-activated protein kinase kinase kinase 7 (MAP3K7)

TANK binding kinase 1

TIRAP

TLR

TNFAIP3

TNFa

TOLLIP

TRAF1

TRAF6

TRIF

TIR domain containing adaptor protein

Toll-like receptors

TNF alpha induced protein 3

tumor necrosis factor $\alpha$

toll interacting protein

TNF receptor associated factor 1

TNF receptor associated factor 6

TIR domain-containing adaptor inducing interferon- $\beta$ 


\section{Introduction}

From birth, our skin is constantly exposed to various microbes, which are capable of transiently or permanently colonizing the cutaneous surfaces and forming the skin microbiota or microbiome. The microbiota has a dual role in our skin: in one hand, plays a crucial role in the maintenance of epidermal homeostasis, on the other hand, if the fine balance between the microbiota and skin cells are disturbed, dysbiosis may occur, and various members of this community have an impact in the pathogenesis of different skin diseases.

\subsection{Skin anatomy and function}

The skin is one of our largest organs, and its main function is to separate our body from the external environment. Because of its special anatomic structure and cell composition, it provides a complex mechanical, chemical and immunological barrier. Apart from that, the skin also has a role in temperature regulation, sensation, and protection against excessive water loss, UV light and pathogenic microbes (1).

The skin is composed of three layers (from top to bottom), the epidermis, dermis and the hypodermis (Figure 1.). The major cellular components of the epidermis are keratinocytes, forming a multilayered
structure. Apart from
keratinocytes, melanin-
producing melanocytes,
mechanosensitive Merkel
cells, and tissue-resident
macrophages, like
Langerhans cells are also
present in the epidermis.

The dermis is connected to the epidermis by a basement membrane and consists of

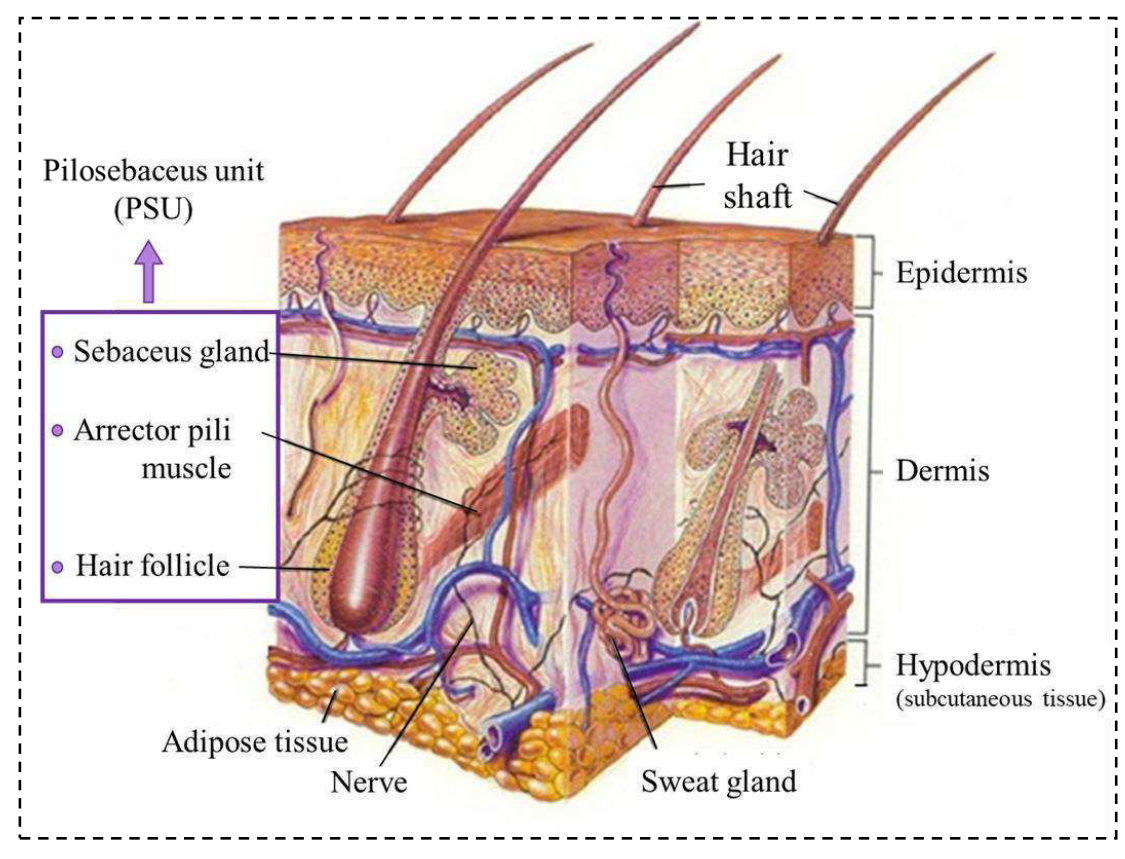

Figure 1. The anatomical structure of the skin connective tissue. It contains hair follicles, different glands (sebaceous, sweat and apocrine glands), nerve endings, lymphatic and blood vessels. 
The deepest layer of the skin is the hypodermis or subcutaneous tissue. It lies below the dermis and composed of loose connective tissue. It contains adipocytes, fibroblasts, and macrophages. Its main function is to serve as energy storage and plays a role in thermoregulation (2).

\subsection{Localization and composition of the cutaneous microbiota}

The cutaneous microbiota populates the epidermis, and the specialized anatomical and functional organelles called pilosebaceous units (PSUs) or follicles of the human skin $(3,4)$. To date, approximately 1000 bacterial species belonging to 19 phyla, as well as fungal (dermatophytes) and viral species have been identified as members of this community (15). Actinobacteria (Cutibacterium and Corynebacterium species), Proteobacteria, Firmicutes (Staphylococcus species) and Bacteroidetes are the most common representatives of the four dominant bacterium phyla (3,5). At a species level, Staphylococcus epidermidis ( $S$. epidermidis) and Cutibacterium acnes (C. acnes, formerly known as Propionibacterium acnes, P. acnes) are the most abundant and common members $(6,7)$.

The human body has regional alterations in terms of $\mathrm{pH}$, temperature, moisture, and the fine anatomic structure of the skin (thickness, sebaceous gland density), and these variations also influence the local composition of the cutaneous microbial communities. For example, in sebaceous gland rich skin regions, such as the back, face, and chest, the predominant bacterial species are the Gram-positive Cutibacterium spp, whereas moist areas favor the growth of Staphylococcus and Corynebacterium spp. Dry areas have the most complex bacterial composition, with representatives from all four bacterial phyla (3,5,8-10). Apart from these variations, many other individual lifestyle and environmental factors shape the microbial diversity of our skin, among others, the mode of birth, age, gender, geographical and socioeconomic properties (11).

\subsection{The role of cutaneous microbiota in healthy skin and the pathogenesis of different skin diseases}

The cutaneous microbiota contributes to the formation and maintenance of skin homeostasis in different ways. Resident microbes inhibit the growth of pathogenic microbes by competing for nutrients and inhabitant space and by the production of antimicrobial compounds(12). For example, $C$. acnes, which is one of the most common members of the skin's resident microbial community exhibits a complex effect. It produces different 
compounds with bacteriostatic and/or antimicrobial properties, including acnecin and shortchain fatty acids (SCFAs). The latter ones can control the growth of pathogenic microbes, such as the methicillin-resistant Staphylococcus aureus (S. aureus) bacterium and also inhibits the commensal S. epidermidis $(13,14)$. On the other hand, the presence of the microbiota also leads to immune and inflammation activation of the host. As a consequence, the affected cell types release various cytokines, chemokines and small host molecules exhibiting antimicrobial properties, called antimicrobial peptides (AMPs), including human $\beta$ defensins (hBDs) (15). The continuous presence of the skin microbiota keeps our immune system at a basal activated state, which helps to induce a rapid and stronger immune activation upon pathogenic attacks $(12,16)$.

Various members of the microbiota are also important in the pathogenesis of different skin diseases. When they develop, microbial dysbiosis leads to exaggerated innate immune and inflammatory events. Examples of such diseases, where different members of the cutaneous microbiota play an important etiopathogenic role include Malassezia spp. in seborrheic dermatitis, $S$ aureus in atopic dermatitis and C. acnes in acne vulgaris $(11,17)$.

\subsection{Skin microbiota-induced innate immune activation in keratinocytes}

Keratinocytes are the major cellular components of the epidermis, and this particular cell type forms a continuous and direct contact with the different members of the cutaneous microbiota (5). Keratinocytes are immunocompetent cells, they can sense danger signals, known as pattern- or damage-associated molecular patterns (PAMPs and DAMPs), through their pattern recognition receptors (PRRs) $(18,19)$. To date, the most well-known and studied PRRs are the Toll-like receptors (TLRs). PAMPs and DAMPs released by human and microbial cells upon insults or injury induce TLR activation, and subsequent activation of innate immune and inflammatory responses in keratinocytes $(19,20)$. 


\subsubsection{C. acnes recognition by human epidermal keratinocytes and their role in acne pathogenesis}

Epidermal keratinocytes recognize the presence of $C$. acnes bacterium in their environment through the activation of TLR2 and TLR4, induction of the canonical TLR signaling pathway, and subsequent innate immune and inflammatory events $(21,22)$. Important mediators of this cascade are the nuclear factor kappa-light-chain-enhancer of activated B cells (NF- $\kappa B$ ) transcription factor, c-Jun N-terminal kinase (JNK), p38 mitogenactivated protein kinases (p38) and extracellular signal-regulated kinases 1 and 2 (ERK1/2) mitogen-activated protein kinases (MAPKs), which regulate the expression of key genes, playing a role in the initialization and execution of downstream responses (23-25). These genes include different cytokines, such as tumor necrosis factor $\alpha(\mathrm{TNF} \alpha)$, interleukin (IL)-1 $\alpha$, IL-1 $\beta$, and IL-6, chemokines, including the IL-8 and C-C motif chemokine ligand 5 (CCL5), antibacterial peptides, such as human beta-defensin 2 (hBD2), and other inflammatory mediators $(26,27)$. The innate immune activation of keratinocytes and the inflammatory milieu they generate in their environment favors the activation of other cell types, including sebocytes, dendritic cells, and macrophages. Adaptive immune events are also induced, leading to the activation of the Th1/Th17 pathway $(28,29)$. Overall, these events contribute to the induction of the characteristic inflammatory symptoms during acne vulgaris pathogenesis in adolescents (Figure 2).

Apart from the microbial component, several intrinsic and extrinsic factors also contribute to the pathogenesis of the diseases. Such intrinsic factor is the hormonal changes during puberty (mainly androgen excess), and as a consequence, the appearance of abnormal keratinocyte and sebocyte functions (enhanced epidermal keratinocyte proliferation, differentiation and increased sebum production of sebocytes) (30). Genetic predisposing factors, e.g. single nucleotide polymorphisms (SNPs) in different genes may also modify an individual risk to develop more severe forms of the disease (31). 

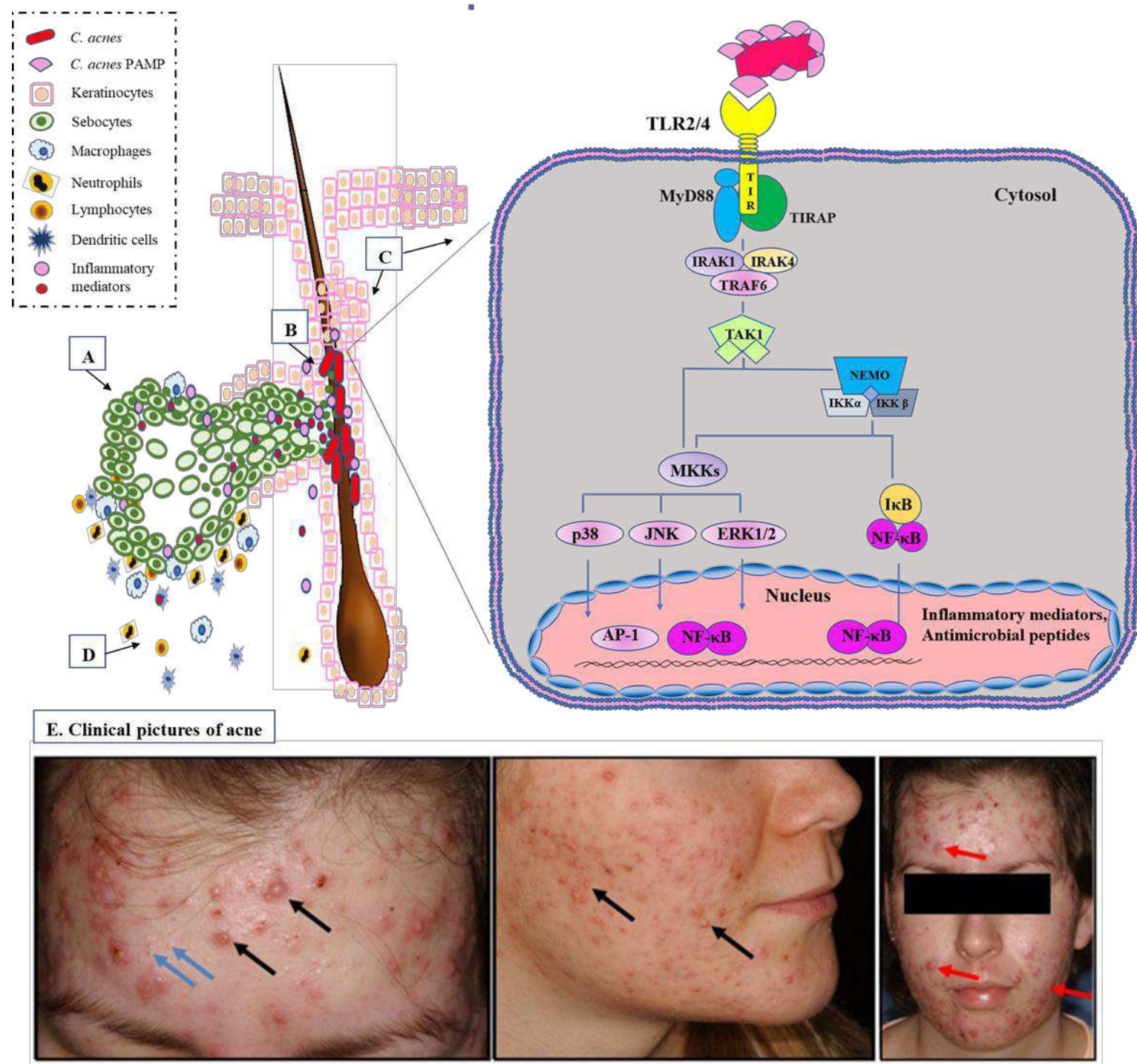

Figure 2. C. acnes-induced TLR signaling pathways in epidermal keratinocytes. (A) Increased sebum production of sebocytes, as a consequence of hormonal changes during puberty, favors (B) C. acnes proliferation. (C) Enhanced keratinocyte proliferation and differentiation can lead to keratinocyte hyperkeratosis. C. acnes recognition by TLR2 and TLR4 induces the canonical NFKB-dependent signaling pathways, increases the production of several cytokines and chemokines, antimicrobial peptides and other inflammatory mediators. (D) The generated inflammatory milieu favors the recruitment and the activation of other cell types, such as professional immune cells. Overall, these events contribute to the induction of the characteristic inflammatory symptoms (E) (comedos-blue arrows, papules- black arrows, pustules, and cysts- red arrows) during acne vulgaris pathogenesis. (Photos were selected from the collection of the Department of Dermatology and Allergology, University of Szeged.) 


\subsection{Factors contributing to the negative regulation of TLR signaling pathways}

Inflammation and acne lesions are generally present transiently throughout life. In adolescents, the inflamed follicles heal by themselves and the affected individuals often do not exhibit any residual signs. After the resolution of the disease, C. acnes bacterium still dominates the microflora, especially in the sebum-rich skin regions, but the bacterium does not usually provoke immune activation and inflammation in keratinocytes and/or other immune cells $(8,16,32)$. This age-dependent response to $C$. acnes indicates the existence of different mechanisms controlling the bacterium induced immune events, but currently, the exact nature of these regulatory mechanisms is still not known (33).

Control of innate immune activation is the key to avoid excessive inflammation and tissue damage. Several negative regulators of the TLR signaling pathways have been identified in the past decade, which act at different levels of the cascade $(34,35)$. TLR pathways are suppressed, among others, by receptor interference (e.g., soluble TLRs, single Ig and TIR domain containing - SIGIRR and interleukin 1 receptor like 1 - IL1RL1), inhibition and destabilization of adaptor complex formation (e.g., SIGIRR, TNFAIP3 interacting protein 1TNIP1, a short form of MYD88 innate immune signal transduction adaptor - MYD88, toll interacting protein - TOLLIP), by dampening crucial enzymatic modification steps (e.g., TNF alpha induced protein 3 - TNFAIP3, interleukin 1 receptor-associated kinase 3 - Irak3, suppressor of cytokine signaling 1 - SOCS1) or by transcriptional regulation (miRNAs, including MIR146A, MIR155, MIR199) (36,37).

\subsubsection{SIGIRR, TOLLIP, TNFAIP3 and TNIP1}

Currently, it is not clear whether and how, and exactly which negative regulators control the human microbiome-induced downstream events. Thus, in our studies we selected SIGIRR, TOLLIP, TNIP1, and TNFAIP3, acting at different levels of the TLR signaling cascade (Figure 3).

SIGIRR is an orphan receptor, composed of a single extracellular Ig domain, a transmembrane domain, a cytoplasmic two amino acid modified TIR domain and a long tail. It controls the activation of different TLR, and the Interleukin 1 Receptor Type 1 (IL-1R1) signaling pathways by competing with the formation of MyD88 dimers, through their TIR domains. As a result, the activation of downstream signaling molecules (IRAK and TRAF6) is reduced, leading to NF- $\kappa \mathrm{B}$ and JNK inhibition $(38,39)$. Through this, SIGIRR plays 
important roles in the regulation of inflammation, cell cycle, differentiation and cell homeostasis (40). Its role in the microbiota-induced processes is also suggested, SIGIRR deficient mice were found to be susceptible to intestinal Salmonella typhimurium colonization, parallel with the loss of commensal microbes in the intestine (41).

TOLLIP is another negative regulator of TLR and IL-R1 signaling pathways. By direct interaction with TLR2 and TLR4, it inhibits downstream signaling and prevents the subsequent inflammatory responses $(42,43)$. It blocks the auto-phosphorylation of IRAK1 and IRAK-2 by direct binding, thus dampens the activation of NF- $\kappa B$ and the production of inflammatory cytokines (42). Consequentially, TOLLIP facilitates the clearance of cellular stress molecules and helps to restore cellular homeostasis (44).

TNFAIP3, also known as A20, is an ubiquitin editing enzyme. Through its zinc finger domains, a ubiquitin ligase, and deubiquitinase activity, TNFAIP3 controls TLR and TNFR pathways at different levels (45). TNFAIP3 acts directly on RIP1 and NEMO, which causes the termination of NF- $\mathrm{B}$ activation, and subsequently dampens the downstream signaling cascades (46). TNFAIP3 functions in human keratinocytes, and appears to be a general regulator of immune and inflammatory cascades in response to different ligands, for example, poly-I:C and imiquimod (TLR3 and TLR7 ligands, respectively) (47,48). A recent study also suggested that TNFAIP3 may help S. epidermidis to persist as a commensal on the human skin, through the control of bacterium-induced NF- $\kappa$ B activation, IL-1 $\beta$ and hBD2 production in keratinocytes (49).

TNIP1, also known as ABIN1, was identified as a TNFAIP3 interacting partner. It can affect different signaling pathways by interacting directly with various proteins, including NEMO, TRAF1, p105, FADD, and RIP1. As a consequence, the TLR-MYD88 signaling cascade and the NF- $\mathrm{KB}$ transcription factor is inhibited, and apoptotic and autoimmune events are negatively affected $(50,51)$. TNIP1 is also expressed in keratinocytes, where this protein controls cell proliferation partly due to the regulation of ERK1/2 signaling cascades (52), and its attenuation sensitizes HaCaT keratinocytes to synthetic TLR ligand treatments (53). 


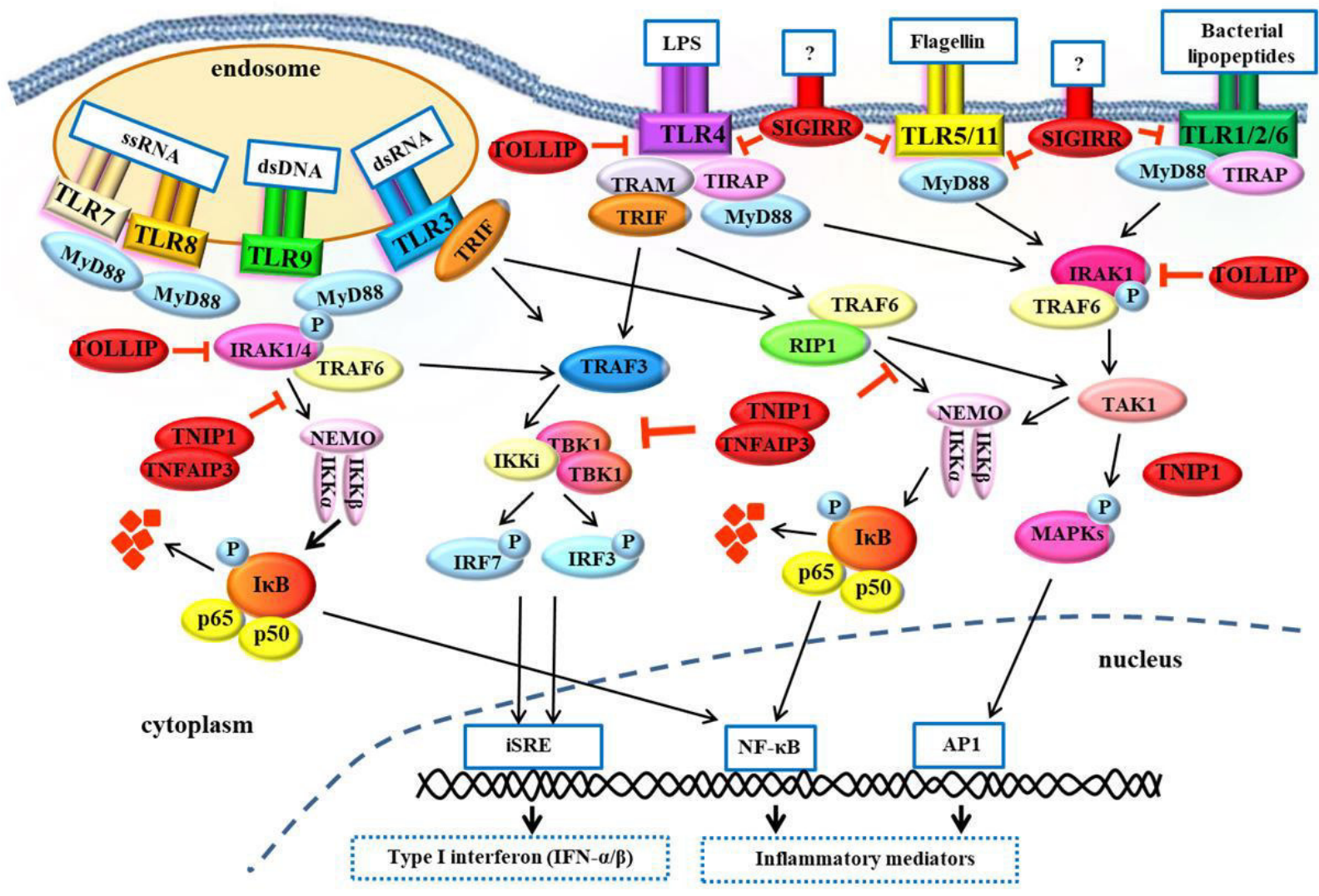

Figure 3. Negative regulation of TLR signaling pathways by SIGIRR, TOLLIP, TNFAIP3 and TNIP1. Adapted from Zhu and Mohan, Mediators Inflamm, 2010. (54). (SIGIRR, TOLLIP, TNFAIP3 and TNIP1 are highlighted with red.) 


\section{Aims}

Our main objective was to identify factors and signaling pathways which play a regulatory role in the microbiota, especially $C$. acnes-induced innate immune activation in human epidermal keratinocytes.

For that, we aimed to analyze well-known negative regulators of TLR signaling pathways, including SIGIRR, TOLLIP, TNFAIP3, and TNIP1:

- whether their expression changes in response to C. acnes in keratinocytes,

- if selected $C$. acnes strains, belonging to various phylogenetic groups within the species (889: 1A, 6609: 1B, ATCC 11828: II), differentially alter the expression of these factors,

- whether and how their expression changes modify the $C$. acnes-induced innate immune and inflammatory events in a human immortalized keratinocyte cell line, HPV-KER,

- and exactly how the expression of these factors is regulated in keratinocytes. 


\section{Materials and Method}

\subsection{Cell cultures and models}

The human immortalized keratinocyte cell line, HPV-KER, was used for our experiments (55). Cells were cultured in keratinocyte serum-free medium (KSFM, Life Technologies, Carlsbad, USA) containing 1\% antibiotic/antimycotic (AB/AM) (Sigma Aldrich, St. Louis, MO, USA) solution and supplemented with epidermal growth factor and brain pituitary extract under standard laboratory conditions $\left(37^{\circ} \mathrm{C}\right.$ in a humidified atmosphere containing $5 \%$ $\left.\mathrm{v} / \mathrm{v} \mathrm{CO}_{2}\right)$.

Normal human epidermal keratinocytes (NHEK) and ex vivo skin biopsies were taken from skin specimens obtained from the Plastic Surgery Unit of our Department. Written informed consent was obtained from investigated individuals. The study was approved by the Human Investigation Review Board of the University of Szeged (PSO-EDAFN-002, 34/2015, 3517, 23 February 2015, Szeged, Hungary) and complied with the ethical standards of research and was in accordance with the Helsinki Declaration.

NHEK cells were isolated from skin samples. Briefly, the adipose tissue was trimmed away and the epidermis and dermis were separated by overnight dispase digestion (Roche Diagnostics, Manheim, Germany) at $4{ }^{\circ} \mathrm{C}$. Keratinocytes were isolated with $0.25 \%$ trypsin (Sigma Aldrich, St. Louis, MO, USA), cultured in a T75 flask and used at the third passage for subsequent experiments.

For ex vivo organotypic skin (OS) cultures, $1 \mathrm{~cm} \mathrm{x} 1 \mathrm{~cm}$ biopsies were washed first with normal saline solution (NSS) containing $2 \% \mathrm{AB} / \mathrm{AM}$, followed by a wash with $\mathrm{AB} / \mathrm{AM}$-free NSS. Subsequently, the biopsies were placed onto the upper chamber of Transwell ${ }^{\circledR}$ Inserts (Corning, New York, USA) and kept at the air-liquid interphase. The dermal part of the biopsies was in contact with DMEM F12 liquid culture medium (Lonza, Basel, Switzerland) supplemented with 10\% FBS (EuroClone, Milan, Italy) lacking AB/AM.

\subsection{Treatments}

For bacterial treatment, HPV-KER and NHEK cells were plated in AB/AM-free KSFM culture medium and co-cultured with live $C$. acnes strains belonging to different phylogenetic groups within the species (889, I/A, ATCC 11828, II, 6609, I/B) for various time using different multiplicity of infection (MOI). Ex vivo skin models were treated with the $C$. acnes 
889 strain at a density of $3 \times 10^{7}$ colony-forming units (cfu) per $\mathrm{cm}^{2}$ for 24 hours. C. acnes were cultured on pre-reduced Columbia agar base supplemented with $5 \%(\mathrm{v} / \mathrm{v})$ bovine blood, vitamin $\mathrm{K} 1$ and haemin (Oxoid, UK). Bacteria were grown at $37{ }^{\circ} \mathrm{C}$ under anaerobic conditions. Single bacteria colonies were inoculated in brain heart infusion (BHI, pH 7.4; Oxoid, UK) and incubated at $37^{\circ} \mathrm{C}$ for 48 hours. After centrifugation (2600 g, 10 minutes, $4{ }^{\circ} \mathrm{C}$ ) and supernatant discard, C. acnes were resuspended in PBS and the number of bacterial cells was measured with a density at $600 \mathrm{~nm}$. The number of bacterial cells was adjusted to 1 $\times 10^{9} \mathrm{cfu} / \mathrm{ml}$ with PBS and aliquoted and stored at $-80^{\circ} \mathrm{C}$ until further use. Every experiment was performed with fresh aliquots.

To analyze the effect of the active form of retinoic acid, ATRA was dissolved in DMSO and a $10^{-6} \mathrm{M}$ concentration was applied to HPV-KER cells for $48 \mathrm{~h}$ before $C$. acnes challenge. ATRA was applied to OSs at a $1.5 \times 10^{-6} \mathrm{M}$ concentration for $24 \mathrm{~h}$. As a control, cells were subjected to DMSO treatment without the active ingredient.

The selective inhibitors of JNK (sp 600125, $10 \mu \mathrm{M}$ ), NF-kB (Bay 11-7085, $10 \mu \mathrm{M}$ ), p38 (sb 203580, $10 \mu \mathrm{M}$ ), ERK1/2 (PD 098059, $20 \mu \mathrm{M}$ ), STAT1 (Fludarabine, $25 \mu \mathrm{M}$ ) and STAT3 (Stattic, $5 \mu \mathrm{M}$ ) or, as a control, DMSO were applied to the cells for 1 hour (all reagents from Sigma Aldrich, St. Louis, MO, USA).

\subsection{Transfection, plasmids and siRNA-mediated gene silencing}

Transient transfection experiments were performed using the X-tremeGENE 9 DNA Transfection Reagent (Roche, Indiana, USA). For the overexpression studies, HPV-KER cells were plated in 12-well plates $(100,000$ cells/well), transfected for 24 hours with $0.5 \mu \mathrm{g}$ empty pcDNA3.1 vector or pcDNA3.1-TNIP1 vector into which TNIP1 cDNA sequences (OriGene Technologies, Inc., MD, USA) had been inserted. Transfection-grade plasmid was prepared using the E.Z.N.A Endo-free plasmid DNA Maxi Kit (Omega Bio-tek, Inc., GA, USA).

For transient siRNA-mediated gene silencing, siRNA was delivered by the Santa Cruz siRNA Transfection Reagent (Santa Cruz Biotechnology, Texas, USA) according to the manufacturer's instructions. 25 nM ON-TARGETplus SMARTpool TNFAIP3-siRNA, $10 \mathrm{nM}$ TNIP1-siRNA, SIGIRR-siRNA, TOLLIP si-RNA or ON-TARGETplus Non-targeting Pool (Dharmacon, Lafayette, USA) constructs were used at the same concentration for 48 hours.

NF- $\kappa \mathrm{B}$ luciferase reporter assay was performed using the PathDetect $\mathrm{pNF}-\kappa \mathrm{B}-\mathrm{Luc}$ CisReporter Plasmid (Stratagene, California, USA), pGL4.75[hRluc/CMV] vector, which was 
transfected with X-tremeGENE 9 DNA Transfection Reagent (Roche, Indiana, USA). For analysis, the Firefly \& Renilla Dual Luciferase Assay Kit (Biotium Inc, California, USA) was used according to the manufacturer's instructions.

\subsection{ELISA}

C. acnes-treated and control HPV-KER cell culture supernatants were collected and levels of secreted IL-8, IL-6 and CCL5 were measured by enzyme-linked immunosorbent assay (ELISA, PeproTech EC Ltd., London, UK) according to the manufactures' instructions.

\subsection{RNA isolation, cDNA synthesis and real-time RT-PCR}

Total RNA from HPV-KER, NHEK cells or the epidermal part of OS cultures were isolated using TRI-Reagent (Molecular Research Center; Cincinnati, USA). cDNA synthesis was performed using $1 \mu \mathrm{g}$ RNA with the iScript TM cDNA Synthesis kit (Bio-Rad, Hercules, USA). Changes in mRNA expression were detected by real-time RT-PCR using the Universal Probe Library (Roche, Indiana, USA) or the TaqMan Gene Expression Assay (Thermo Scientific, Rockford, USA). Table 1. lists the PCR protocols and primer sequences used. All data were normalized to the $18 \mathrm{~S}$ rRNA using the $\Delta \Delta \mathrm{C}_{\mathrm{t}}$ method and compared to the timematched untreated control samples.

\begin{tabular}{|c|c|c|c|c|c|c|}
\hline \multirow{2}{*}{ Gene } & \multirow{2}{*}{ Primer sequences } & \multirow{2}{*}{ Probe } & \multicolumn{4}{|c|}{ PCR protocol } \\
\hline & & & Denaturation & Annealing & Extension & Cycle \\
\hline 18S rRNA & $\begin{array}{l}\text { FWD: CGCTCCACCAACTAAGAACG } \\
\text { REV: CTCAACACGGGAAACCTCAC }\end{array}$ & 77 & \multirow{13}{*}{$95^{\circ} \mathrm{C}$} & \multirow{11}{*}{$58^{\circ} \mathrm{C}$} & \multirow{13}{*}{$37^{\circ} \mathrm{C}$} & \multirow{13}{*}{$40 \mathrm{x}$} \\
\hline TNFAIP3 & $\begin{array}{l}\text { FWD: TGCACACTGTGTTTCATCGAG } \\
\text { REV: ACGCTGTGGGACTGACTTTC } \\
\end{array}$ & 74 & & & & \\
\hline TNIP1 & $\begin{array}{l}\text { FWD: TGGTCACGCAGAATGAGTTG } \\
\text { REV: CTCCCTCTGGAAGTCCTCCT }\end{array}$ & 62 & & & & \\
\hline SIGIRR & $\begin{array}{l}\text { FWD: CTCAGAGCCATGCCAGGT } \\
\text { REV: CCTCAGCACCTGGTCTTCA }\end{array}$ & 55 & & & & \\
\hline TOLLIP & \begin{tabular}{|l|} 
FWD:TCCCCGCTGGAATAAGGT \\
REV: CGTCCATGGAGAAGGCTCT \\
\end{tabular} & 86 & & & & \\
\hline IL-6 & $\begin{array}{l}\text { FWD: CAGGAGCCCAGCTATGAACT } \\
\text { REV: GAAGGCAGCAGGCAACAC }\end{array}$ & 45 & & & & \\
\hline IL-1A & $\begin{array}{l}\text { FWD: GGTTGAGTTTAAGCCAATCCA } \\
\text { REV: TGCTGACCTAGGCTTGATGA }\end{array}$ & 6 & & & & \\
\hline CCL5 & $\begin{array}{l}\text { FWD: TGCCCACATCAAGGAGTATTT } \\
\text { REV: CTTTCGGGTGACAAAGACG }\end{array}$ & 59 & & & & \\
\hline TLR2 & $\begin{array}{l}\text { FWD: CTCTCGGTGTCGGAATGTC } \\
\text { REV: AGGATCAGCAGGAACAGAGC }\end{array}$ & 56 & & & & \\
\hline TLR3 & $\begin{array}{l}\text { FWD: AGAGTTGTCATCGAATCAAATTAAAG } \\
\text { REV: AATCTTCCAATTGCGTGAAAA }\end{array}$ & 80 & & & & \\
\hline TLR4 & $\begin{array}{l}\text { FWD: GCCTGATTTCATTTATATGAGGTTCTA } \\
\text { REV: GACAACTCCCTATTTCCTCATTTCT }\end{array}$ & 69 & & & & \\
\hline TNFA & \multirow{2}{*}{\multicolumn{2}{|c|}{$\begin{array}{l}\text { Taq Man Genexpression assay } \\
\text { Hs00174128_m1 } \\
\text { Taq Man Genexpression assay } \\
\text { Hs001743103 }\end{array}$}} & & \multirow{2}{*}{$60^{\circ} \mathrm{C}$} & & \\
\hline IL-8 & & & & & & \\
\hline
\end{tabular}

Table 1. Realtime RT-PCR primers and the applied protocol. 


\subsection{Protein isolation and western blot analysis}

For the preparation of whole cell lysates, samples were collected and lysed in lysis buffer containing $20 \mathrm{mM}$ 4-(2-hydroxyethyl)-1-piperazineethanesulfonic acid, $150 \mathrm{mM} \mathrm{KCl,} 1 \mathrm{mM}$ $\mathrm{MgCl}_{2}, 1 \mathrm{mM}$ DTT, 5\% Triton X-100, 10\% glycerol, 0.1\% NP-40, 1\% Protease Inhibitor Cocktail, phenylmethylsulfonyl fluoride and 0.5\% SDS (all from Sigma Aldrich, St. Louis, MO, USA). Protein concentrations were measured with the BCA Protein Assay Kit (Thermo Scientific, Rockford, USA). Samples $(30 \mu \mathrm{g})$ were separated on a 7.5\% SDS-PAGE gel and transferred to nitrocellulose membrane (Bio-Rad, Hercules, USA), blocked in Tris-buffered saline containing 5\% non-fat dried milk. Membranes were incubated overnight at $4{ }^{\circ} \mathrm{C}$ with primary anti-TNFAIP3 antibody diluted 1:1000 (Abcam, Cambridge, United Kingdom), antiSIGIRR antibody diluted 1:1000, anti-TOLLIP antibody diluted 1:1000 anti-TNIP1 antibody diluted 1:500 and anti-actin (Sigma Aldrich, St. Louis, MO, USA) diluted 1:1000. Subsequently, membranes were incubated for 1 hour at room temperature with horseradish peroxidase- or alkali-phosphatase-conjugated anti-rabbit or anti-mouse $\operatorname{IgG}$ secondary antibody (Santa Cruz Biotechnology, Texas, USA) diluted 1:2000. Proteins were visualized with 5-Bromo-4-chloro-3-indolyl phosphate p-toluidine salt (BCIP) (Sigma Aldrich, St. Louis, MO, USA) or with luminol (Bio-Rad, Hercules, USA) using an Omega Lum ${ }^{\mathrm{TM}}$ G Imaging System (Gel Company, CA, USA) and quantified using Image Pro Plus Software.

\subsection{Fluorescence microscopic analysis}

HPV-KER cells were grown on glass sides, fixed with $2 \%$ paraformaldehyde (PFA) for 5 minutes, permeabilized with $0.1 \%$ Triton X, 2\% PFA-containing phosphate-buffered saline (PBS), and blocked for 2 hours at room temperature (RT) with PBS containing 1\% bovine serum albumin (BSA), $0.05 \%$ Triton $\mathrm{X}$ 100, and 10\% goat serum. Cells were stained overnight at $4{ }^{\circ} \mathrm{C}$ with anti-human TNIP1 antibody (Sigma Aldrich, St. Louis, MO, USA) or rabbit IgG for isotype control. As a secondary antibody, Alexa Fluor 488 conjugated antirabbit $\mathrm{IgG}$, was used for 2 hours at RT. Filamentous actin was stained by Alexa Fluor $546^{\circledR}$ phalloidin (Life Technologies, Carlsbad, USA) diluted 1:100 in PBS containing 1\% BSA for 20 minutes. Nuclei were stained for 10 minutes with 4',6-diamidino-2-phenylindole (DAPI) diluted 1:500. TNIP1 were visualized with Zeiss Axio Imager Z1fluorescence microscope.

Frozen sections of ex vivo skin models were pre-incubated with PBS for 5 minutes and fixed and permeabilized with Foxp3 staining buffer set (Thermo Scientific, Rockford, USA) 
and blocked for 1 hour at RT with Tris-buffered saline (TBS) containing 1\% BSA and 1\% normal goat serum (NGS) (Sigma Aldrich, St. Louis, MO, USA). Cells were stained for 1 hour with anti-human TNIP1 antibody or rabbit IgG for isotype control. As a secondary antibody, Alexa Fluor 546 conjugated anti-rabbit IgG (Thermo Scientific, Rockford, USA) was used for 1 hour at RT. Nuclei were stained for 6 minutes with 4',6-diamidino-2phenylindole (DAPI) diluted 1:100. TNFAIP3 was visualized using a ZEISS LSM 880 Confocal Laser Scanning Microscope (Zeiss, Oberkochen, Germany), magnification: 63x.

For visualization of TNFAIP3, frozen sections of ex vivo OS models were pre-incubated with PBS for 5 minutes, fixed with 4\% paraformaldehyde for 5 minutes and permeabilized with $0.25 \%$ TritonX-100 (Thermo Scientific, Rockford, USA) containing TBS for 10 minutes. For blocking, TBS containing 5\% FBS and 5\% NGS was used for 1 hour at RT. Cells were stained for 1 hour with anti-human TNFAIP3 antibody diluted 1:300 or rabbit IgG (Santa Cruz Biotechnology, Texas, USA) for isotype control. As a secondary antibody, Alexa Fluor 546 conjugated anti-rabbit IgG (Thermo Scientific, Rockford, USA) was used for 1 hour at RT. Nuclei were stained for 5 minutes with DAPI diluted 1:100. TNFAIP3 was visualized using a ZEISS LSM 880 Confocal Laser Scanning Microscope (Zeiss, Oberkochen, Germany) magnification: $63 x$.

\subsection{Data analysis}

TNFAIP3 gene expression was analyzed using publicly available microarray data from the GEO Profile Database (https://www.ncbi.nlm.nih.gov/geoprofiles/33444972, GDS2478 datasets, ID: 33444972), which compares the total RNA content of healthy skin ( $n=6)$ with lesional $(n=6)$ and non-lesional $(n=6)$ samples from acne patients (56).

\subsection{Statistical analysis}

Unless otherwise noted, all data are presented as mean \pm standard error of the mean (SEM) of three independent experiments. For real time RT-PCR analyzes and ELISA, each treatment was performed at least in triplicate; for western blot and fluorescence microscopic analysis, each treatment was performed once in every independent experiment. Data were compared using paired, two-sample t-test with Holm-Bonferroni or false discovery rate (FDR) correction using RStudio. A probability value of less than 0.05 was considered significant. 


\section{Results}

We aimed to systematically analyze SIGIRR, TOLLIP, TNFAIP3 and TNIP1 mRNA and protein expression changes in response to C. acnes bacterium and studied their possible role in the bacterium-induced innate immune activation of keratinocytes.

\subsection{Studying the expression levels and the possible role of SIGIRR in the C. acnes- induced innate immune activation of keratinocytes}

We analyzed SIGIRR expression changes in human in vitro cultured keratinocytes cocultured with the $C$. acnes 889 strain $(\mathrm{MOI}=100)$. The applied $C$. acnes dose was determined in extensive preliminary studies. We chose conditions that induced relatively fast and reproducible cellular responses but did not induce cell death in the time course of our studies (55). We found that SIGIRR is expressed in HPV-KER cells, but its mRNA expression remained unchanged after $C$. acnes treatment (Figure 4).

It has been suggested that various $C$. acnes strains may have different effects on the cellular and molecular properties of human keratinocytes $(27,55,57)$. We applied $C$. acnes strains, belonging to various phylogenetic groups within the species (C. acnes 889, 6609, ATCC 11828, Group IA, IB and II.) (MOI=100) and compared the effects. None of the applied strains induced SIGIRR mRNA expression changes at the used bacterial doses (Figure 4/A, B, C).

To analyze whether SIGIRR expression depended on the used C. acnes doses, we applied the bacterium in different MOIs $(25,100,300)$ for 6 hours, but SIGIRR mRNA expression did not change during the time-course of the experiment (Figure 4/D).

We also studied SIGIRR protein expression changes upon treatment with the C. acnes 889 strain. Similarly to the mRNA results, its protein levels remained the same in the presence of the bacterium. 
A

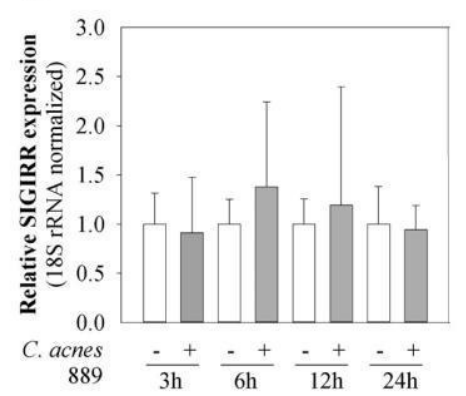

D

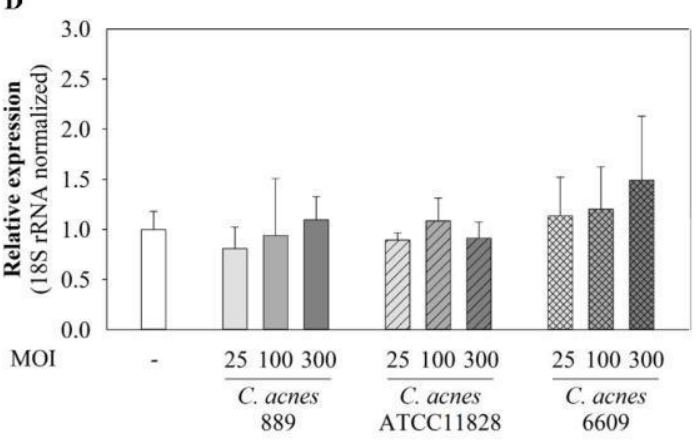

B

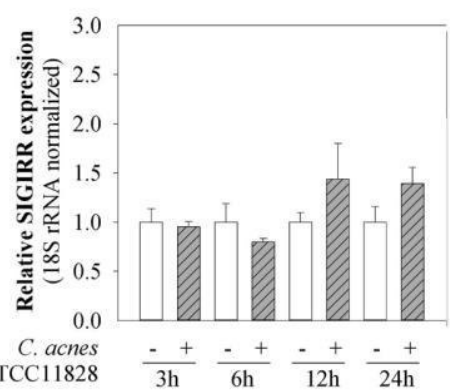

E
C

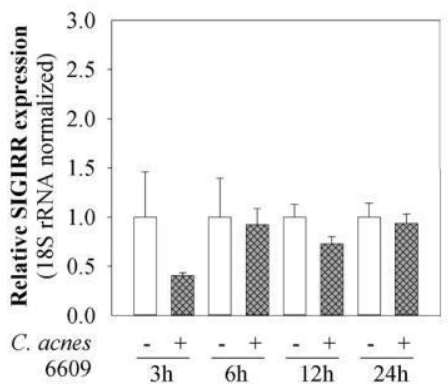

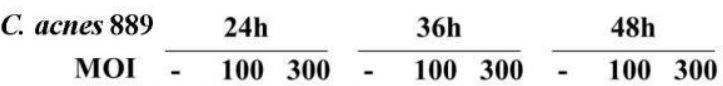

Figure 4. SIGIRR mRNA and protein expression levels do not change in HPV-KER cells, co-cultured with selected C. acnes strains. HPV-KER cells were treated with the . acnes 889, ATCC11828, and 6609 strains (MOI=100, unless otherwise indicated). (A-D) SIGIRR mRNA expression changes were studied by realtime RT-PCR. Error bars are SD, representative experiment. (E) SIGIRR protein was detected with western blot analysis. Representative blot.

Next, we also tested whether changes in SIGIRR levels modify the $C$. acnes-induced innate immune and inflammatory events in HPV-KER cells. For that, transient siRNAmediated silencing was performed, and $C$. acnes-induced TNF $\alpha$ mRNA expression changes were monitored by real-time RT-PCR. Silencing of SIGIRR resulted in a $60 \%$ decrease in the mRNA levels, but neither basal nor the bacterium-induced TNF $\alpha$ levels were affected (Figure 5/A, B). 
A

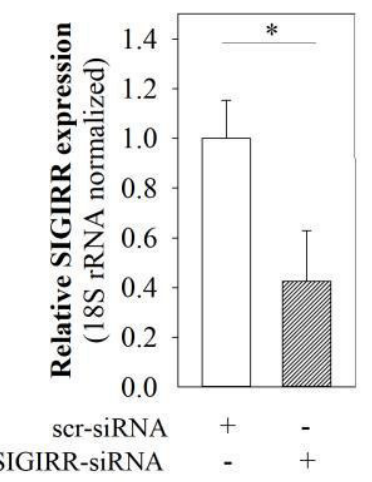

B

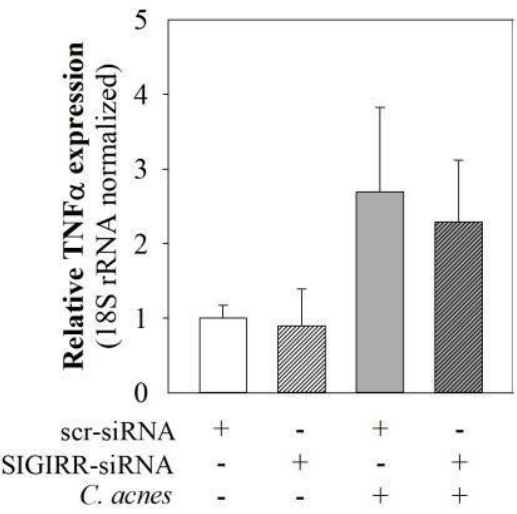

Figure 5. SIGIRR expression changes do not affect TNFa mRNA expression levels. siRNA-mediated silencing (48 hours) of SIGIRR was performed in HPV-KER cells, and they were co-cultured with the C. acnes 889 strain (MOI=100) for 6 hours. (A, B) SIGIRR and TNF $\alpha$ mRNA levels were analyzed by real-time RT-PCR. All data were normalized to $18 \mathrm{~S}$ rRNA and compared to the time-matched untreated samples transfected with scrambled siRNA (scr-siRNA). Error bars are SD, representative experiments. Statistical analysis: paired, twosample t-test, $* \mathrm{p}<0.05$.

Our results suggest that even though SIGIRR is expressed in HPV-KER cells, it does not seem to play important roles in the negative regulation of the $C$. acnes-induced immune and inflammatory processes in keratinocytes.

\subsection{Studying the expression levels and the possible roles of TOLLIP, in the C. acnes- induced innate immune activation of keratinocytes}

Next, we analyzed TOLLIP expression levels in HPV-KER cells upon $C$. acnes treatment. We co-cultured the cells with the $C$. acnes 889, 6609 and ATCC 11828 strains using different bacterial doses and analyzed TOLLIP expression by real-time RT-PCR. We found that TOLLIP mRNA levels remained unchanged using this experimental setup (Figure 6/A, B, C, D). We observed similar results by analyzing TOLLIP protein levels upon C. acnes 889 treatment (Figure 6/E). 


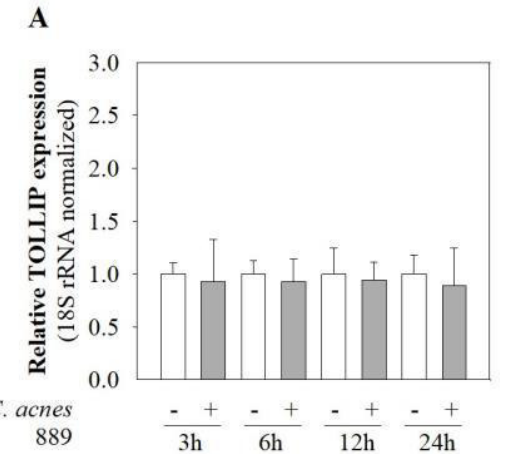

D

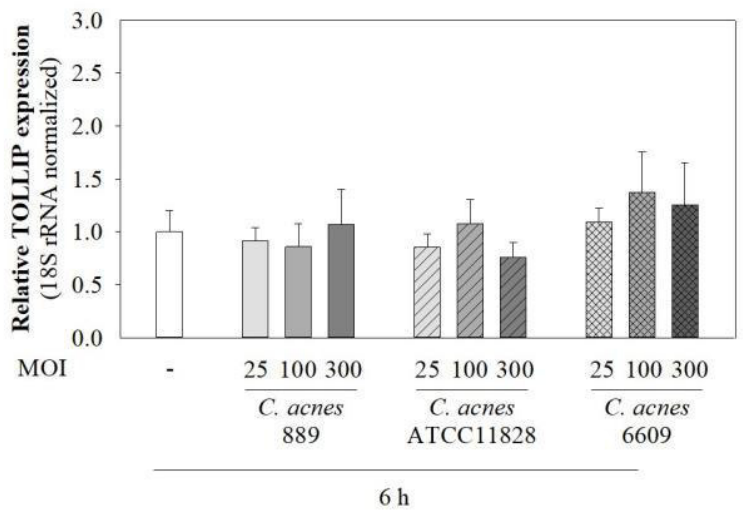

B

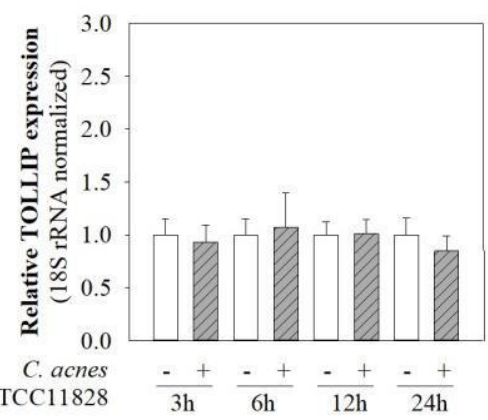

E

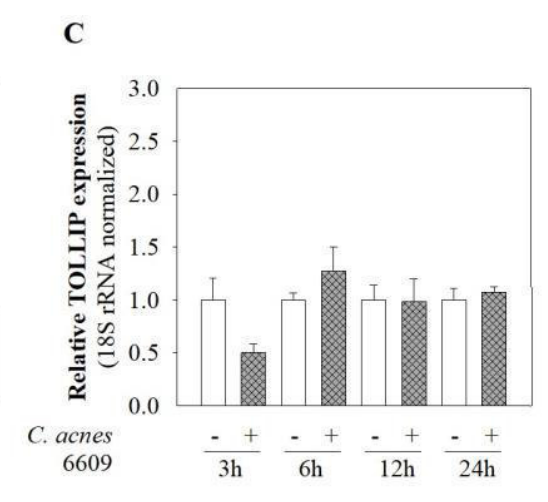

.
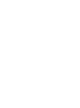

Figure 6. TOLLIP mRNA and protein expression levels do not change in HPV-KER cells, co-cultured with selected $\boldsymbol{C}$. acnes strains. HPV-KER cells were treated with the $C$. acnes 889, ATCC11828, and 6609 strains (MOI=100, unless otherwise indicated). (A-D) TOLLIP mRNA expression changes were studied by realtime RT-PCR. Error bars are SD, representative experiment. (E) TOLLIP protein was detected with western blot analysis. Representative blot.

We also investigated whether changes in TOLLIP levels modify the bacterium-induced TNF $\alpha$ levels in HPV-KER cells. We found that transient, siRNA-mediated silencing of TOLLIP did not influence basal and $C$. acnes-induced TNF $\alpha$ mRNA expression levels (Figure 7/A, B).

Our results suggest that similar to our observation regarding SIGIRR, TOLLIP is expressed in HPV-KER cells, but it does not seem to play important roles in the negative regulation of the $C$. acnes-induced processes in keratinocytes. 
A

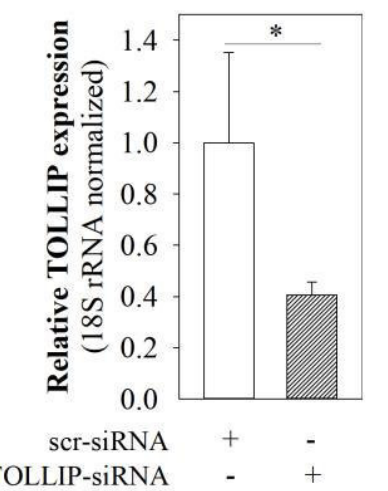

B

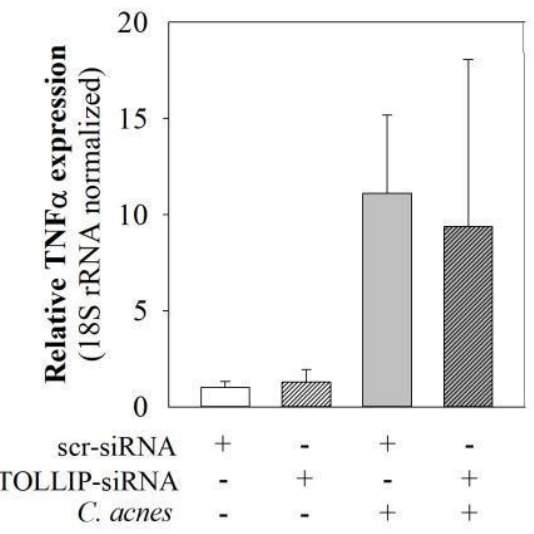

Figure 7. Changes in TOLLIP levels do not affect TNF $\alpha$ mRNA expression. siRNA-mediated silencing (48 hours) of TOLLIP was performed in HPV-KER cells, and they were co-cultured with the C. acnes 889 strain (MOI=100) for 6 hours. (A, B) TOLLIP and TNF $\alpha$ mRNA levels were analyzed by real-time RT-PCR. All data were normalized to $18 \mathrm{~S}$ rRNA and compared to the time-matched untreated samples transfected with scrambled siRNA (scr-siRNA). Error bars are SD, representative experiments. Statistical analysis: paired, two-sample t-test, $* \mathrm{p}<0.05$.

\subsection{Studying the expression levels and the possible role of TNFAIP3 in the $C$. acnes- induced innate immune activation of keratinocytes}

Next, we investigated the possible role of TNFAIP3 in the $C$. acnes-induced innate immune activation of keratinocytes.

Earlier studies have already shown that TNFAIP3 is expressed in the human epidermis (47). We confirmed this finding by immunofluorescence staining in healthy, full-thickness skin biopsy samples, where the TNFAIP3 protein was present throughout the entire epidermis, especially in the more differentiated keratinocyte layers. (Figure 8.)
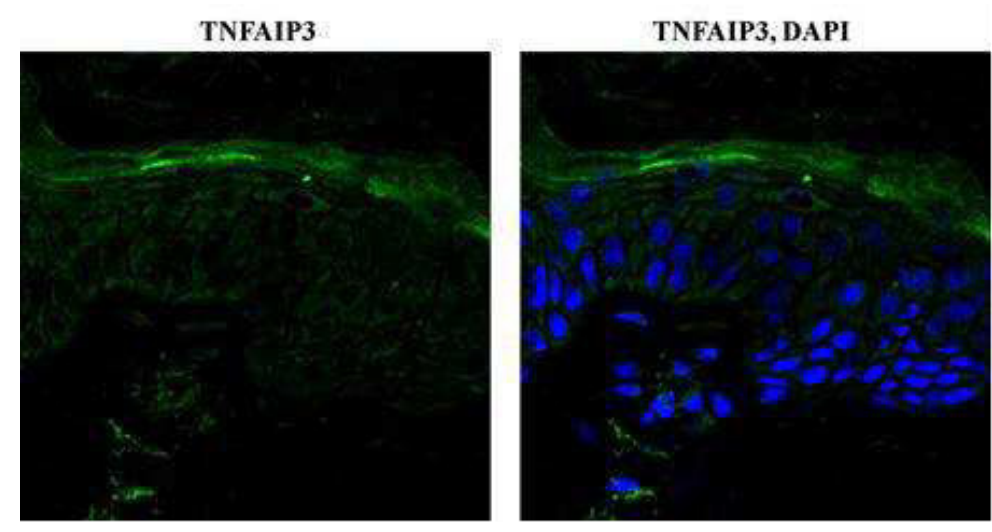

Figure 8. TNFAIP3 expression in the skin. TNFAIP3 protein expression was visualized using immunofluorescence staining for TNFAIP3 (green) and DAPI (blue) in a full-thickness skin biopsy. 


\subsubsection{C. acnes bacterium increases TNFAIP3 expression in keratinocytes}

To analyze whether $C$. acnes affects TNFAIP3 expression levels in keratinocytes, we cocultured HPV-KER cells with the $C$. acnes 889 bacterium strain $(\mathrm{MOI}=100)$ and analyzed the mRNA and protein expression changes with real-time RT-PCR and western blot analysis. We found that TNFAIP3 mRNA levels rapidly and transiently increased in the presence of the bacterium, reaching a maximum at 12 hours after bacterial treatment (Figure 9/A).

A

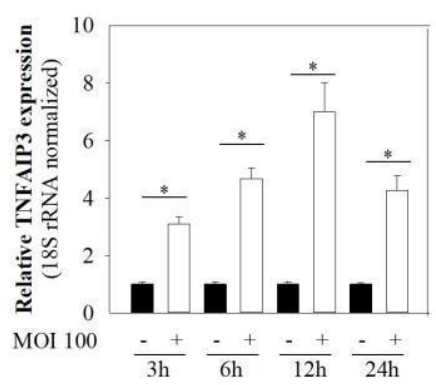

D

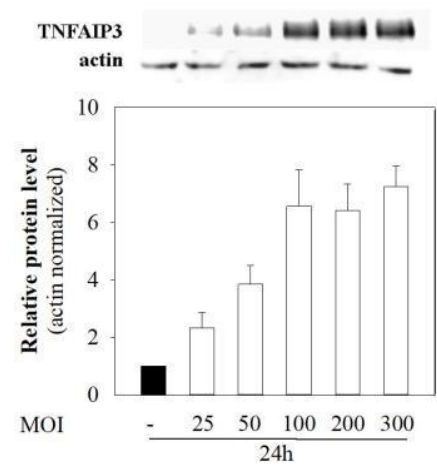

B

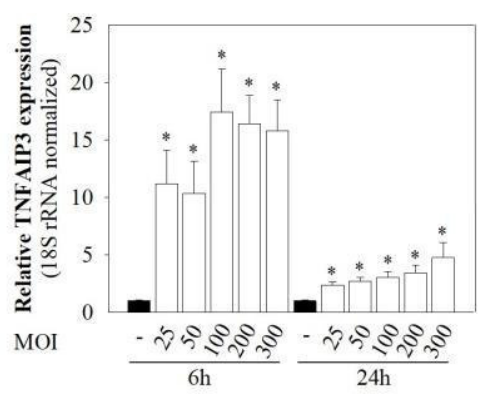

$\mathbf{E}$

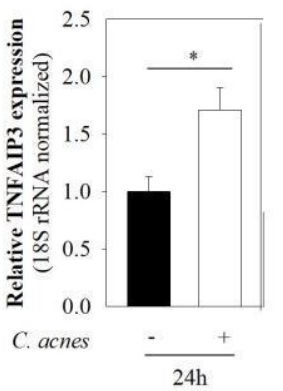

F
C
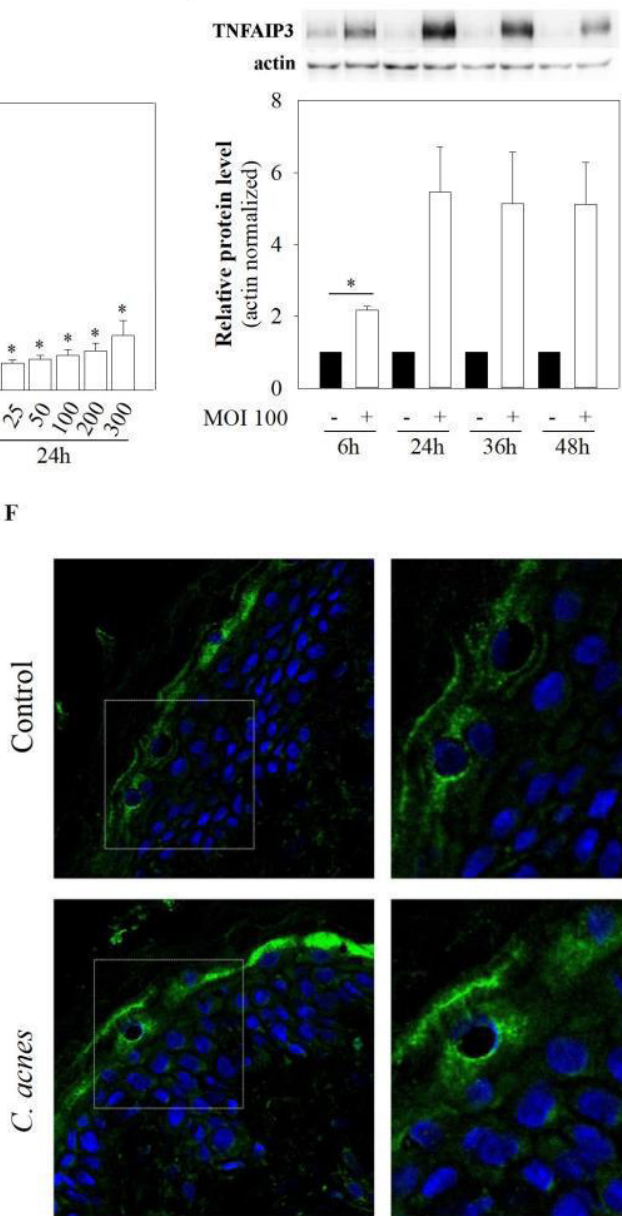

TNFAIP3, DAPI
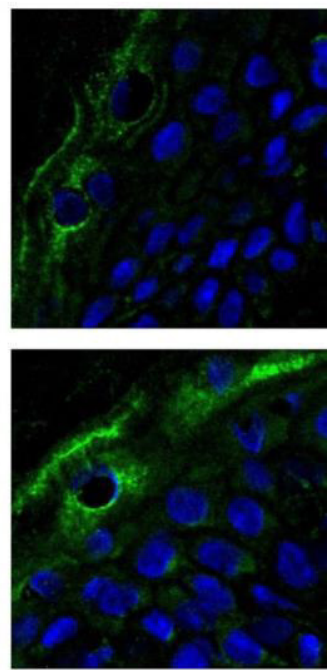

enlarged

Figure 9. $C$. acnes upregulates TNFAIP3 mRNA and protein expression levels. A-D) HPV-KER cells were treated with the $C$. acnes 889 strain (MOI=100, unless otherwise indicated) and mRNA expression changes were analyzed by real-time RT-PCR, whereas protein expression was followed by western blot analysis. (E, F) OS samples were treated with the bacterium for 24 hours. mRNA expression of TNFAIP3 was analyzed only in the epidermis by real-time RT-PCR, and protein expression was visualized using immunofluorescence staining for TNFAIP3 (green) and DAPI (blue) in the full-thickness skin biopsies. mRNA data were normalized to $18 \mathrm{~S}$ rRNA, whereas protein data to actin and compared to the time-matched untreated control samples. Error bars are SE. Statistical analysis: paired, two-sample t-test with Holm-Bonferroni correction: * p<0.05. 
To analyze a possible strain-specific regulation of TNFAIP3, we co-cultured the HPVKER cells with $C$. acnes 6609 and ATCC 11828 strains (MOI=100) and compared their effects. We did not observe any strain-specific differences: TNFAIP3 mRNA expression changes were similar, independent of the strain used (Figure 10).
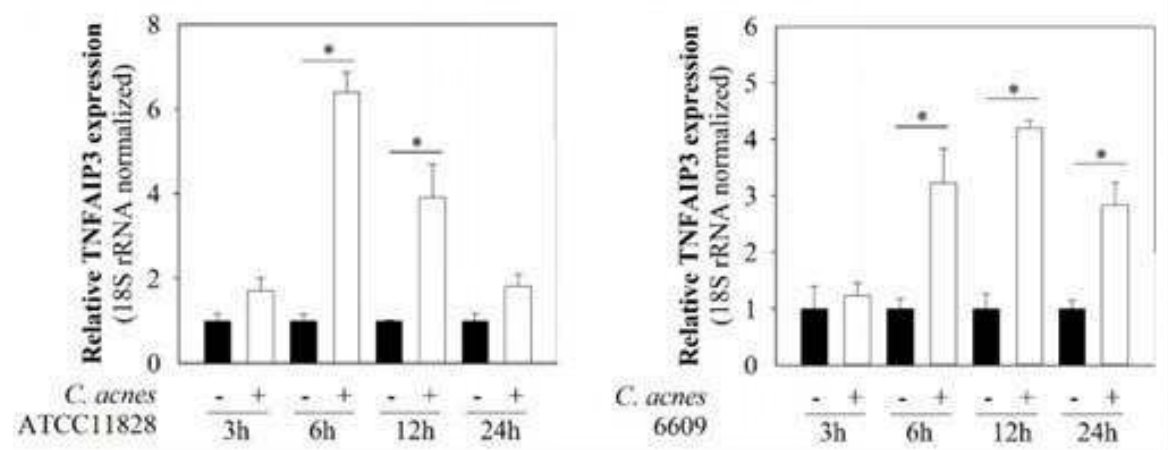

Figure 10. TNFAIP3 expression changes does not differ in HPV-KER cells, co-cultured with different C. acnes strains. HPV-KER cells were treated with C. acnes ATCC11828 or 6609 strains $(\mathrm{MOI}=100)$ and TNFAIP3 mRNA expression was analyzed by real-time RT-PCR (representative experiment). Data were normalized to $18 \mathrm{~S}$ rRNA and compared to the time-matched untreated control values. Error bars are SD. Statistical analysis: paired, two-sample t-test, ${ }^{*} \mathrm{p}<0.05$.

TNFAIP3 protein expression levels also rapidly increased in response to the bacterium $(\mathrm{MOI}=100)$ and remained high during the time-course of our study (Figure 9/C).

We also analyzed whether changes in $C$. acnes-induced TNFAIP3 expression were dosedependent, by co-culturing HPV-KER cells with different MOIs. We found that the extent of TNFAIP3 mRNA and protein expression changes depended on the bacterial dose (Figure 9/B, D).

To confirm that the observed changes were not specific properties of the immortalized keratinocyte cell line used, we performed the experiments with established OS cultures. Ex vivo skin biopsies were treated with live $C$. acnes bacterium and changes in the mRNA expression were analyzed using real-time RT-PCR. TNFAIP3 mRNA expression levels increased in the epidermis part of the OS cultures upon $C$. acnes treatment, similar to what we observed in monolayer cultures. Immunofluorescence microscopy analysis also revealed elevated TNFAIP3 protein levels (Figure 9/E, F). 


\subsubsection{Changes in $C$. acnes-induced TNFAIP3 expression are dependent on JNK and NF-кB signaling pathways}

We investigated the signaling mechanisms playing important roles in TNFAIP3 regulation. The DREAM transcription complex is known to repress its transcription when bound to the DRE3 and DRE4 elements in the TNFAIP3 promoter region in human lung microvessel endothelial cells (HLMVECs). The TNFAIP3 promoter also harbors functional NF- $\kappa B$ binding sites $(58,59)$. To identify the possible signaling pathways that are involved in C. acnes-induced upregulation of TNFAIP3 in keratinocytes, we investigated the contribution of selected pathways playing important roles in the bacterium-induced signaling events (2325). We inhibited the NF- $\kappa \mathrm{B}$ and MAPK (JNK, p38 and ERK1/2) signaling pathways using specific inhibitors and analyzed changes in TNFAIP3 mRNA and protein expression in HPV-KER cells after co-culturing the cells for 12 and 24 hours with the C. acnes 889 strain.

We found that inhibition of the JNK signaling pathway decreased basal TNFAIP3 mRNA expression, whereas C. acnes-induced mRNA (Figure 11/A), and also protein (Figure 11/C) levels were affected by JNK and NF-אB inhibition.

$\mathbf{A}$

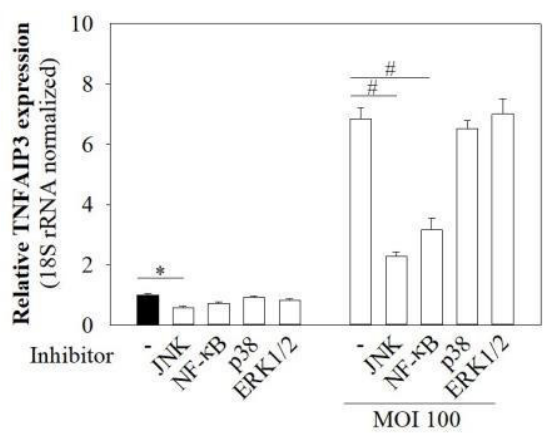

B

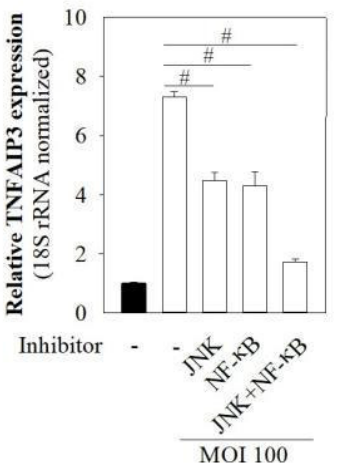

C
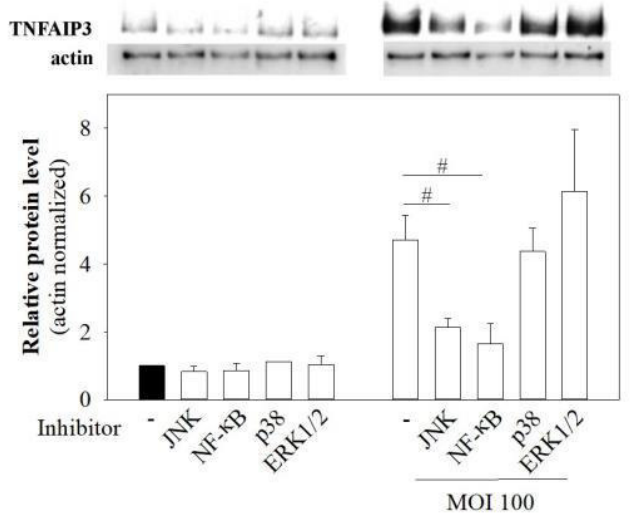

Figure 11. JNK and NF-кB regulates $C$. acnes-induced upregulation of TNFAIP3 expression in keratinocytes. HPV-KER cells were pretreated with selective inhibitors for JNK, NF- $\mathrm{B}$, p38, and ERK1/2 or, as a control, DMSO for 1 hour. (A, B) C. acnes challenge was performed for 12 hours and mRNA expression changes were analyzed by real-time RT-PCR. Data were normalized to $18 \mathrm{~S}$ rRNA. (E) After a 24 hour bacterial treatment, protein expression was monitored by western blot analysis. Data were normalized to actin. All data were compared to time-matched DMSO-treated samples. Statistical analysis: paired, two-sample t-test with Holm-Bonferroni correction, where basal TNFAIP3 expression * $<0.05$ or C. acnes-induced expression \# $\mathrm{p}<0.05$ were compared. 
Dual inhibition of these two signaling pathways almost completely prevented the bacterium-induced changes in mRNA expression (Figure 11/B), suggesting that these two pathways play major roles in the transcriptional regulation of the TNFAIP3 locus.

\subsubsection{TNFAIP3 affects the basal and $C$. acnes-induced levels of inflammatory mediators in keratinocytes}

To analyze the role of TNFAIP3 in the regulation of key inflammatory mediators, siRNAmediated silencing was performed. First, we analyzed the effect of TNFAIP3 on NF-kB activity using a luciferase reporter assay. Silencing resulted in an average knockdown of $40 \%$ of TNFAIP3 protein levels (Figure 12/A). In these samples, the basal NF-кB promoter activity significantly increased, compared to the scrambled-siRNA (scr-siRNA) transfected controls (Figure 12/B).

We also monitored the consequences of these events and found that TNFAIP3 silencing significantly increased the basal mRNA expression of TNF $\alpha$, IL-1 $\alpha$, IL-6, IL-8 and CCL5 and the secreted levels of IL-6 and IL-8, compared to the scr-siRNA control samples. These data suggest that TNFAIP3 may have important roles in the establishment and maintenance of keratinocyte cellular homeostasis by controlling the constitutive expression of different key inflammatory mediators.

We also investigated the effect of TNFAIP3 knockdown on the C. acnes-induced levels of IL-6, IL-8 and CCL5, and found that silencing also significantly increased the levels of these mRNAs and the secreted proteins (Figure 12/C, D). 
A

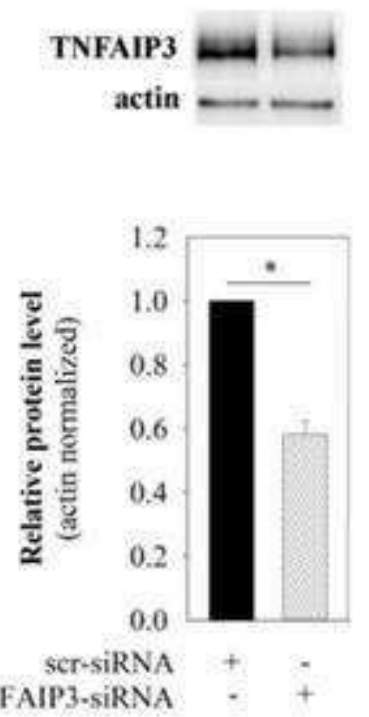

B
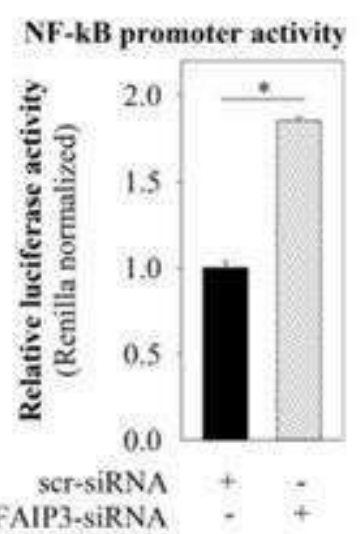

C

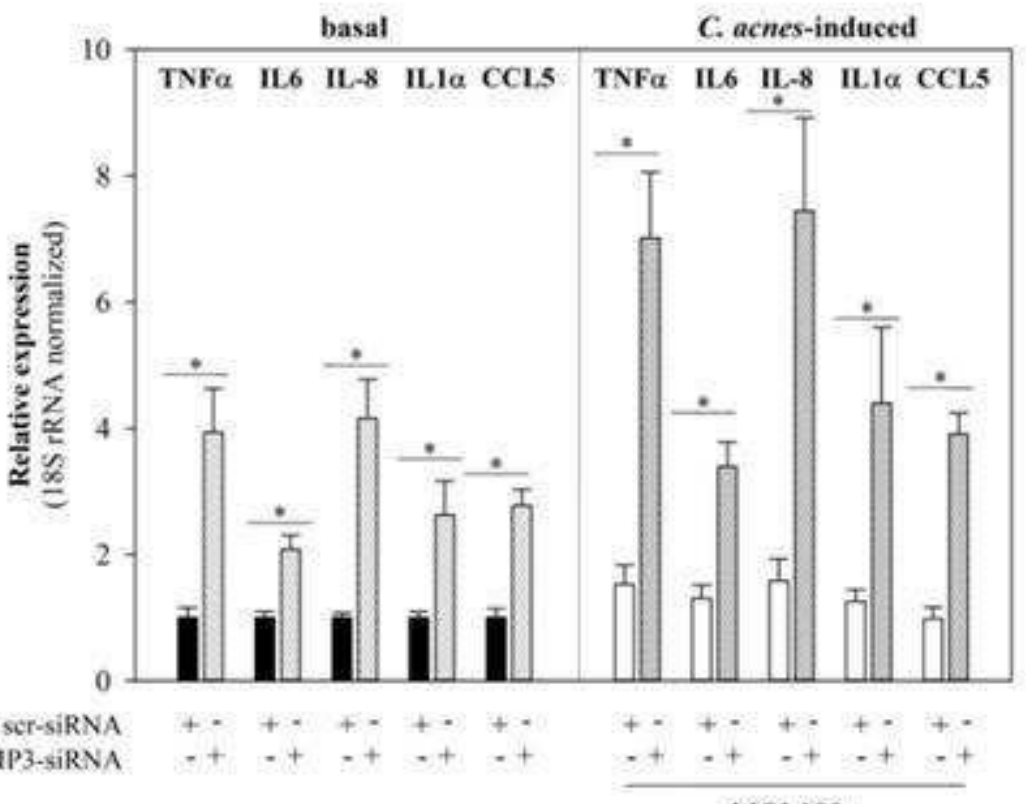

D

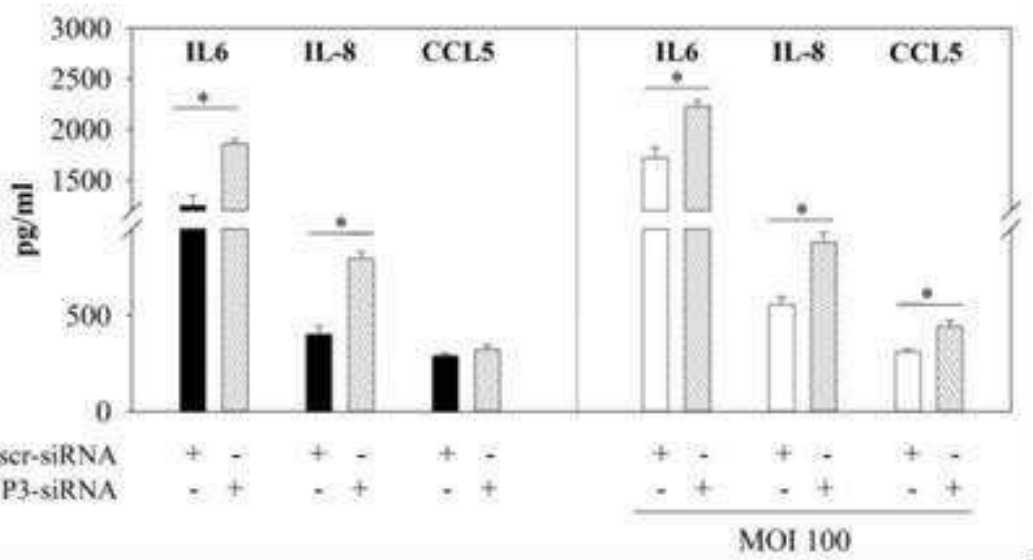

Figure 12. TNFAIP3 negatively regulates downstream targets of the TLR signaling pathways. siRNAmediated silencing of TNFAIP3 (48 hours) was performed in HPV-KER cells followed by a $C$. acnes (MOI=100) treatment. (A) TNFAIP3 protein levels were analyzed by western blotting and quantitated using the

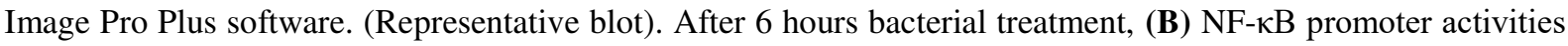
were measured with a luciferase reporter assay, whereas (C) mRNA levels of TNF $\alpha$, IL-1 $\alpha$, IL-6, IL-8, and CCL5 were monitored by real-time RT-PCR. (D) After a 24 hours bacterial treatment, secreted cytokine and chemokine levels were measured by ELISA. In luciferase reporter assay all data were normalized to Renilla activity, in western blot analyzes to actin, whereas in real-time RT-PCR analyzes to 18S rRNA, and compared to the time-matched untreated samples transfected with a scrambled siRNA (scr-siRNA). Error bars are SD (A) or SE (B-D). Statistical analysis: paired, two-sample t-test with Holm-Bonferroni correction: * $\mathrm{p}<0.05$. 


\subsubsection{TNFAIP3 expression differs in acne lesions}

According to our current understanding, C. acnes plays important roles in the regulation and maintenance of epidermal homeostasis, as well as in acne vulgaris pathogenesis as an opportunistic pathogen because of bacterial dysbiosis. To analyze whether TNFAIP3 tissue levels differ in healthy and lesional skin samples, we analyzed data from a publicly available GEO Profile dataset, comparing the total RNA content of healthy individuals with lesional and non-lesional skin samples of acne patients (56). We found significantly increased levels of TNFAIP3 mRNA expression in the lesional skin samples compared to the corresponding non-lesional skin of acne patients (Figure 13.).

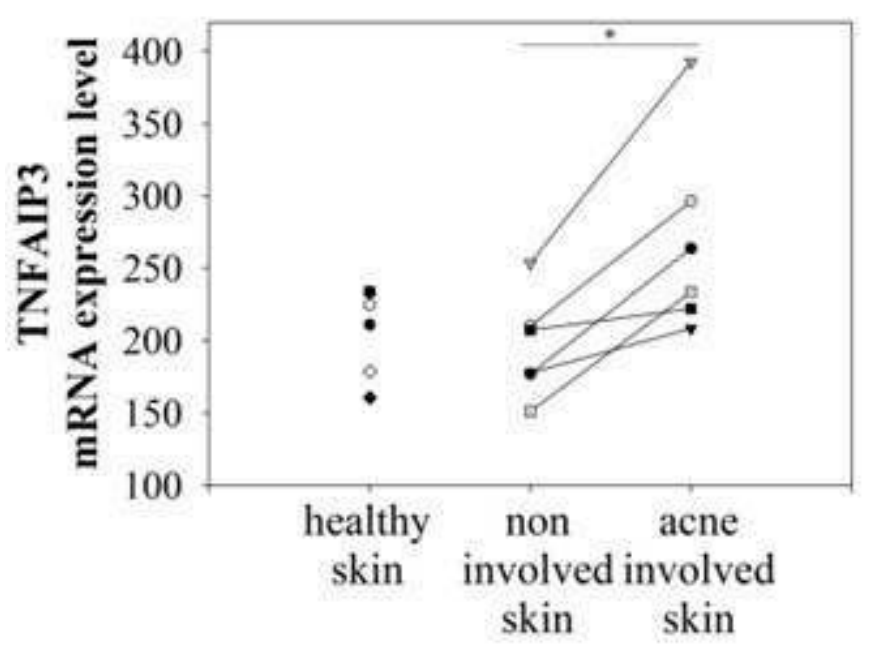

Figure 13. Elevated TNFAIP3 mRNA expression levels are detected in the lesional skin samples of acne patients. TNFAIP3 expression profile from Geo Profile (https://www.ncbi.nlm.nih.gov/geoprofiles/33 444972) data were analyzed and compared with paired student t-test with HolmBonferroni correction, $* \mathrm{p}<0.05$.

\subsection{TNIP1 is expressed in keratinocytes and its expression increases in the presence of C. acnes}

We also analyzed the role of a known interaction partner of TNFAIP3, TNIP1, in the $C$. acnes induced immune events. We monitored its expression changes in HPV-KER cells upon treatment with the bacterium. We found that TNIP1 is expressed in HPV-KER cells and its mRNA expression rapidly and significantly increased after co-culturing the cells with the $C$. acnes 889 strain $(\mathrm{MOI}=100)$, reaching a maximum at 12 to 24 hours (Figure 14/A).

Next, we applied different $C$. acnes strains $(889,6609$, ATCC 11828, MOI=100) and compared their effects, but no strain-specific differences were observed: all of the C. acnes strains induced similar changes in mRNA expression in HPV-KER cells (Figure 14/B). Subsequently, only the $C$. acnes 889 strain was used in further experiments. 
We also found that bacterium-induced changes in TNIP1 expression were dosedependent: the abundance of mRNA increased in parallel with increasing $C$. acnes 889 bacterial doses (Figure 14/C).

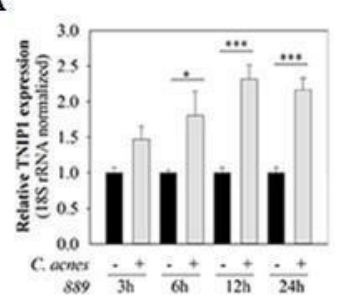

D
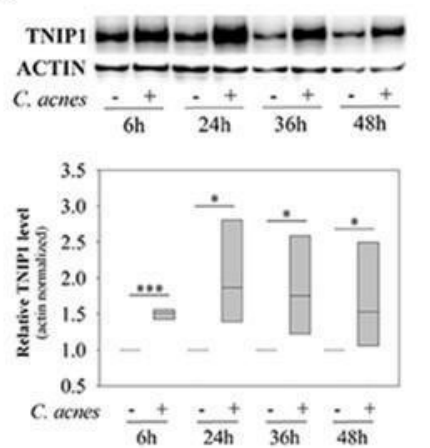

B
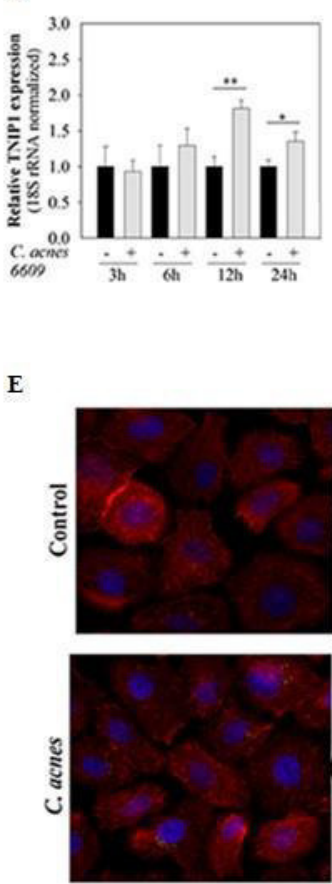
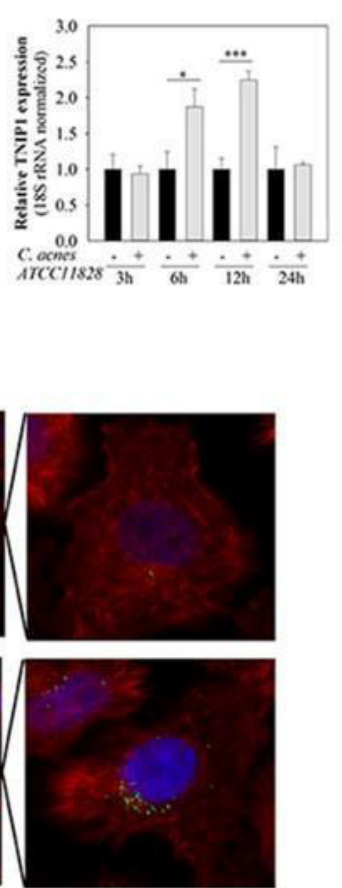

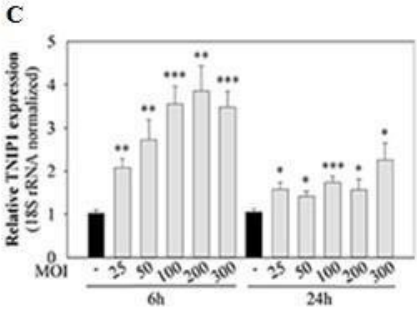

$\mathbf{F}$
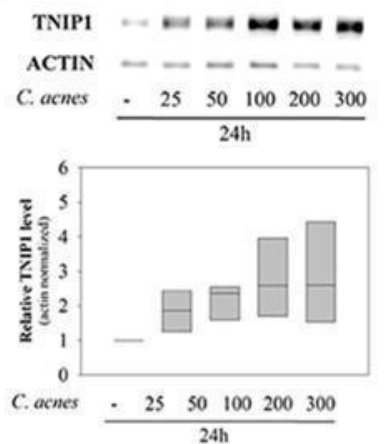

Figure 14. $C$. acnes upregulates TNIP1 mRNA and protein levels in HPV-KER cells. Cells were treated with $C$. acnes 889 (MOI=100, unless otherwise indicated), and changes in mRNA and protein levels were analyzed. (A-C) mRNA expression changes were analyzed by real-time RT-PCR, whereas protein expression $(\mathbf{D}, \mathbf{F})$ was followed by western blot analysis and quantified using Image Pro Plus software or (E) was visualized using immunofluorescence staining for TNIP1 (green), DAPI (blue) and Phalloidin (red). All mRNA data were normalized to $18 \mathrm{~S}$ rRNA, whereas protein levels were normalized to actin and compared to the time-matched untreated control samples. Error bars are SE (A-C) or SD (D, F). In all panels, statistical analysis: paired, twosample t-test with Holm-Bonferroni correction: * $\mathrm{p}<0.05$.

Next, we analyzed TNIP1 protein levels in HPV-KER cells using western blot analysis and immunocytochemistry. Elevated TNIP1 levels were detected in the 6-hour samples and remained high during the time-course of the experiment (Figure 14/D). Immunocytochemical staining of TNIP1 resulted in the presence of immunofluorescent dots, occurring mostly in the perinuclear region of HPV-KER cells. The number of labeled dots increased after 24 hours of $C$. acnes treatment (MOI=100) (Figure 14/E). Similarly to the mRNA levels, the abundance of TNIP1 protein increased in parallel with increasing $C$. acnes 889 doses (Figure 14/F). 


\subsubsection{C. acnes-induced expression changes of TNIP1 are regulated by different signaling pathways in keratinocytes}

To identify which signaling pathways are involved in the regulation of basal and $C$. acnes-induced TNIP1 expression levels in keratinocytes, we treated HPV-KER cells with specific inhibitors of well-known representatives (JNK, NF- $\kappa B$, p38, ERK1/2, STAT1, and STAT3) of different signaling pathways before the $C$. acnes treatment and analyzed subsequent changes in TNIP1 mRNA expression by real-time RT-PCR. We found that constitutive TNIP1 expression was significantly decreased when JNK and ERK1/2 were inhibited. Furthermore, $C$. acnes-induced changes in TNIP1 expression diminished in response to inhibition of JNK and ERK1/2 as well as of NF- $\kappa \mathrm{B}$ and p38. In contrast, no effect was observed with STAT1 and STAT3 inhibition (Figure 15).

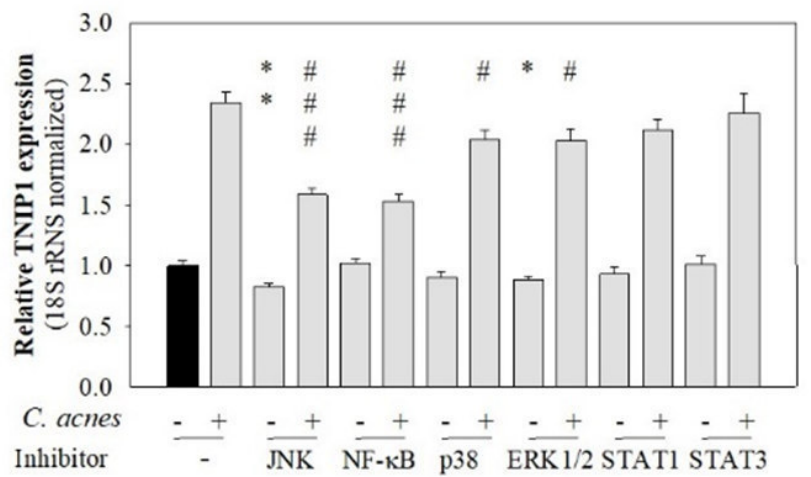

Figure 15. JNK, NF-kB, p38 and ERK1/2 signaling pathways regulate $C$. acnes-induced mRNA levels of TNIP1. HPV-KER cells were pretreated with selective inhibitors for JNK, NF- $\kappa B$, p38, ERK1/2, STAT1 and STAT3 or, as a control, DMSO for 1 hour. C. acnes 889 (MOI=100) challenge was performed for $12 \mathrm{~h}$ and TNIP1 mRNA levels were analyzed by real-time RT-PCR and normalized to $18 \mathrm{~S}$ rRNA. All data were compared to the DMSO-treated control samples. Statistical analysis: paired, two-sample t-test with FDR correction, basal TNIP1 expression *p $<0.05, * * \mathrm{p}<0.01$ or $C$. acnes-induced TNIP1 expression, \#p $<0,05, \# \#$ \# $<0.001$.

\subsubsection{Regulation of TNIP1 levels in response to $C$. acnes bacterium is not specific for HPV-KER cells}

To confirm that $C$. acnes-induced TNIP1 expression changes were not specific to the HPV-KER cell line, we repeated the co-culturing experiments using NHEK cells and found similar results we observed in case of HPV-KER immortalized keratinocytes. TNIP1 mRNA levels increased in response to C. acnes treatment (Figure 16/A), and increased protein levels were also noted in NHEK cell cultures 24 hours after bacterial treatment (Figure 16/B).

We also checked whether the observed results were specific to keratinocyte monolayer cultures, and repeated the experiments using ex vivo, full-thickness skin biopsy samples. Six $\mathrm{mm}$ punch biopsies of healthy donors were cultured at air-liquid interphase and aliquots of 
the $C$. acnes 889 strain were applied to the top (epidermal side) of the samples. We performed an immunofluorescence analysis, which showed that TNIP1 protein was expressed throughout the epidermis and that slightly higher levels were detected in the less differentiated, basal layers. Within the keratinocytes, localization was primarily cytoplasmic and perinuclear. Elevated TNIP1-staining levels were found 24 hours after C. acnes treatment (Figure 16/C).

A

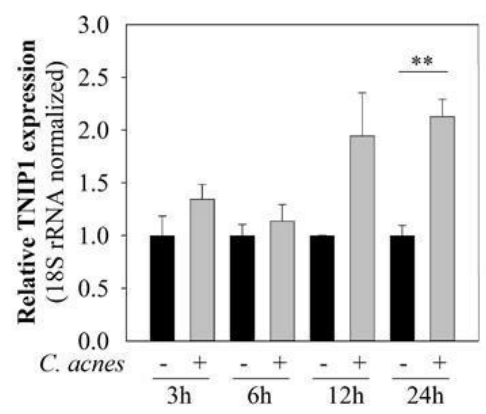

B

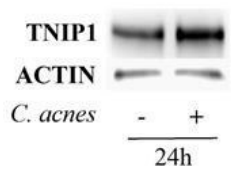

C
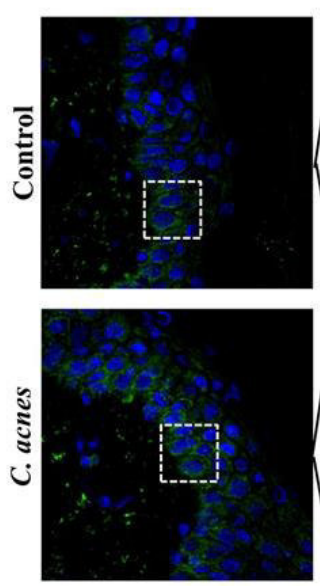
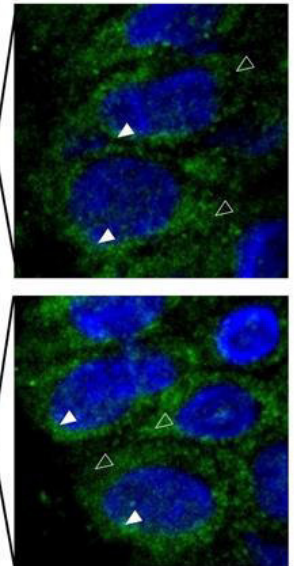

Figure 16. TNIP1 mRNA and protein expression increases in response to $C$. acnes treatment in NHEK cells and ex vivo OS models. NHEK cells were treated with the C. acnes 889 strain (MOI=100) and (A) TNIP1 mRNA expression changes of were analyzed by real-time RT-PCR, whereas protein expression (B) was followed by western blot analysis. mRNA data were normalized to $18 \mathrm{~S}$ rRNA and compared to time-matched untreated samples. (C) OS samples were treated with $3 \times 10^{7}$ bacterium $/ \mathrm{cm}^{2}$ for 24 hours, and cells were visualized using immunofluorescence staining for TNIP1 (green) and DAPI (blue). Empty arrows indicate cytoplasmic location and filled arrows indicated perinuclear localization. Statistical analysis: paired, two-sample t-test with FDR correction: **p $<0.01$.

\subsubsection{TNIP1 down-regulates both constitutive and $C$. acnes-induced inflammatory cytokines and chemokines expression}

To analyze the role of TNIP1 in the regulation of $C$. acnes-induced inflammatory events, we experimentally modified endogenous TNIP1 levels with cDNA-based transient overexpression or siRNA-mediated silencing. We monitored the expression of selected proinflammatory cytokines and chemokines that are known downstream targets of the TLR signaling pathway, as well as the promoter activity of the NF- $\mathrm{BB}$ transcription factor, using real-time RT-PCR, ELISA analysis and a luciferase- reporter assay. 
A

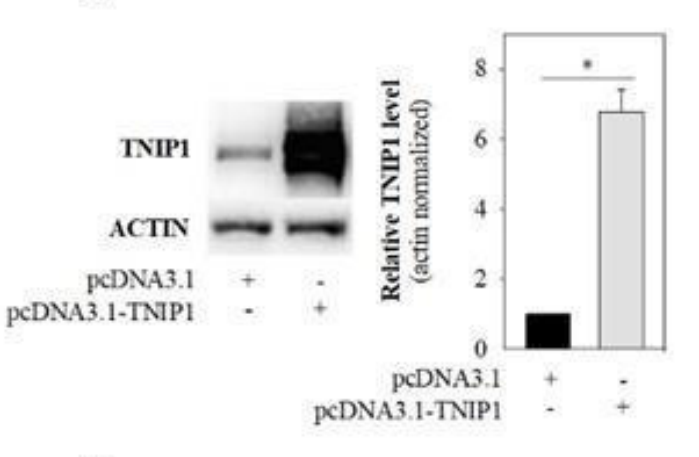

B

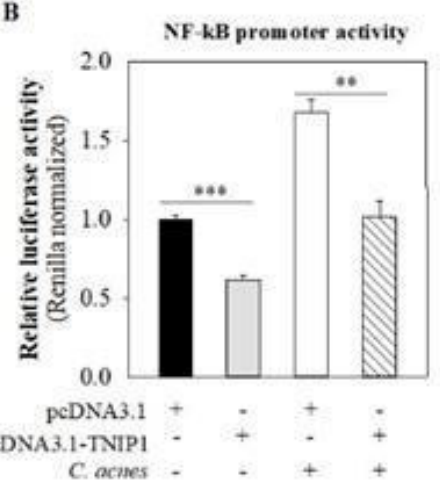

C

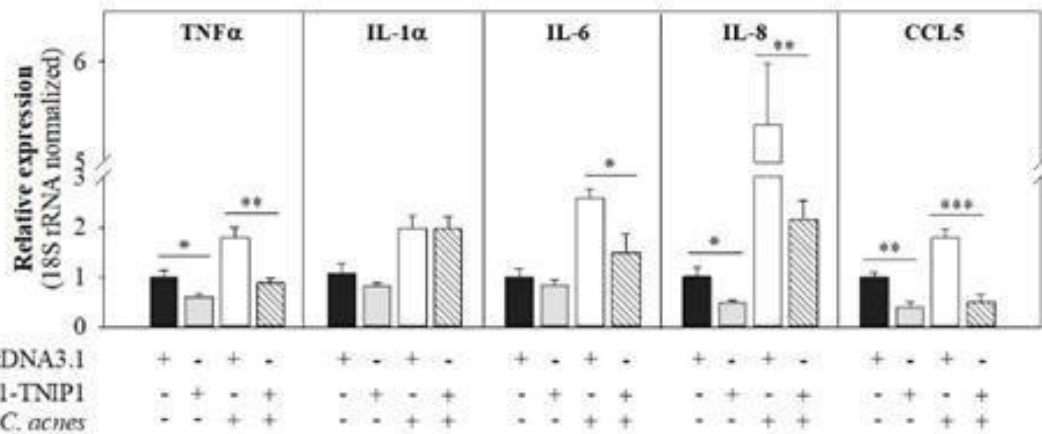

D
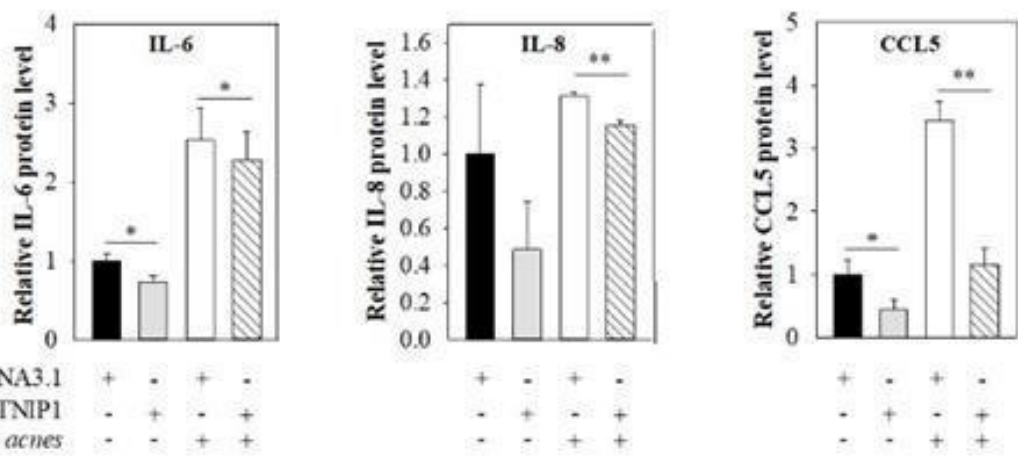

Figure 17. TNIP1 overexpression affects the downstream targets of the TLR signaling pathway. TNIP1 overexpression (24 hours) was performed and HPV-KER cells were treated with C. acnes (MOI=100). (A) TNIP1 protein levels were analyzed by western blot and quantitated using the Image Pro Plus software. (Representative blot.) After 6 hours bacterial treatment, (B) NF- $\mathrm{BB}$ promoter activities were measured with luciferase reporter assay, whereas (C) mRNA levels of TNF $\alpha$, IL-6, IL-8 and CCL5 were monitored by real-time RT-PCR. (D) After 24 hours bacterial treatment, secreted cytokine and chemokine levels were measured by ELISA and relative protein levels are presented. In luciferase reporter assay all data were normalized to Renilla , in western blot analyzes to actin, whereas in real-time RT-PCR analyzes to $18 \mathrm{~S}$ rRNA and compared to the timematched untreated samples transfected with an empty vector (pcDNA3.1). Statistical analysis: paired, twosample t-test: $* \mathrm{p}<0.05, * * \mathrm{p}<0.01, * * * \mathrm{p}<0,001$. 
A

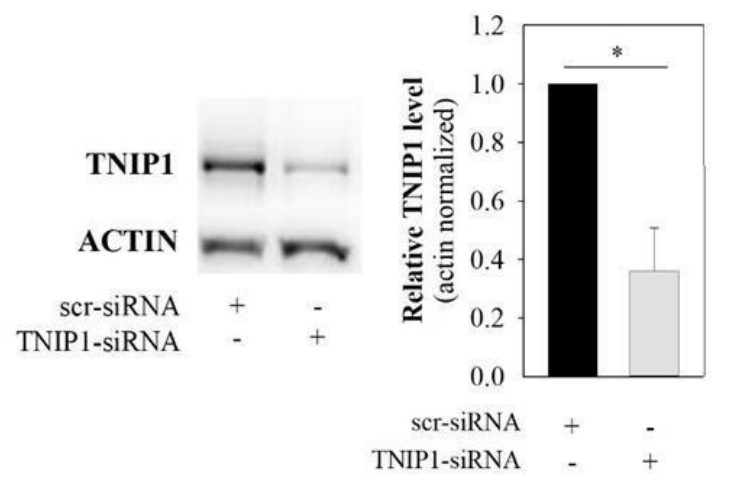

C

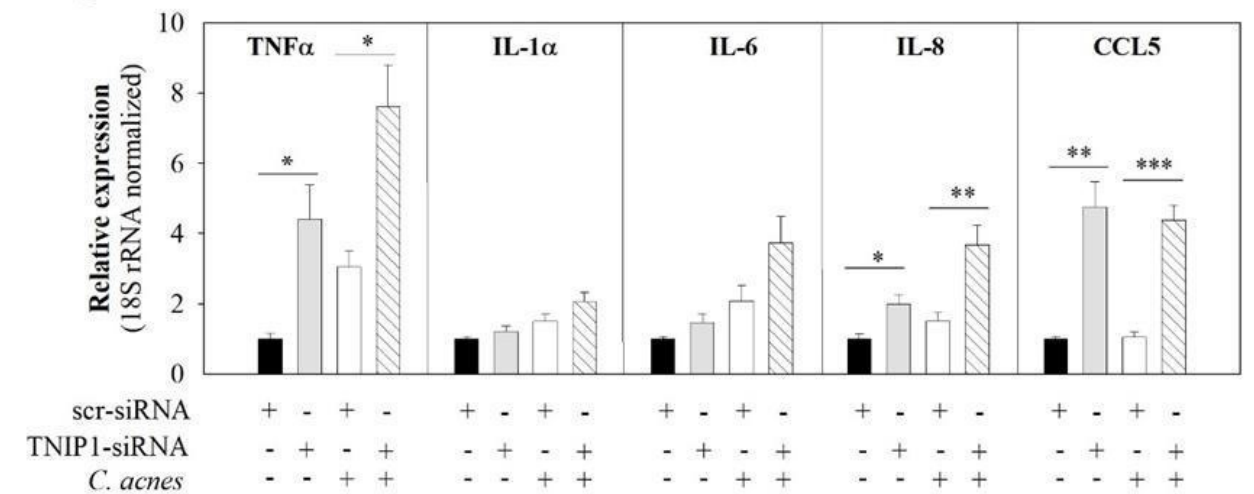

D

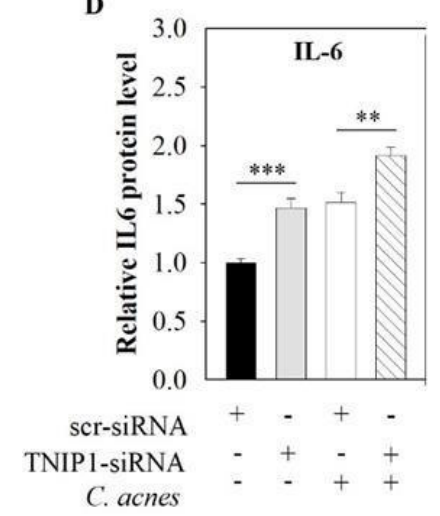

B

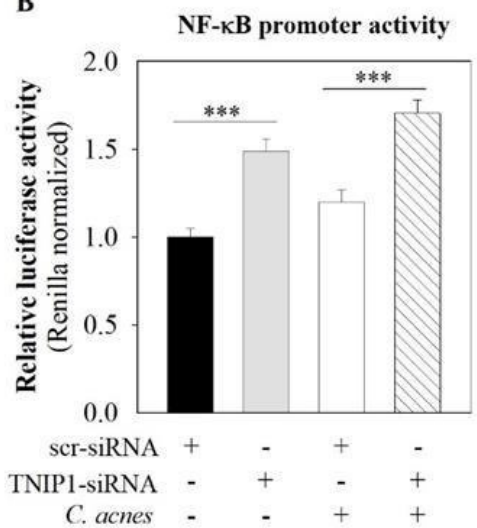

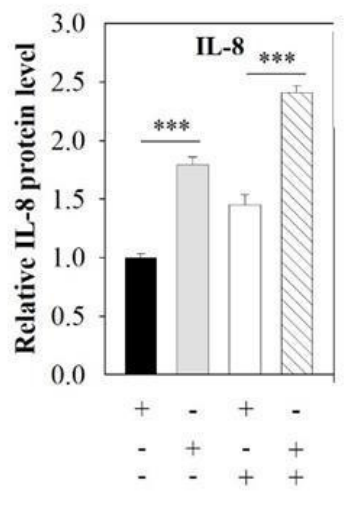

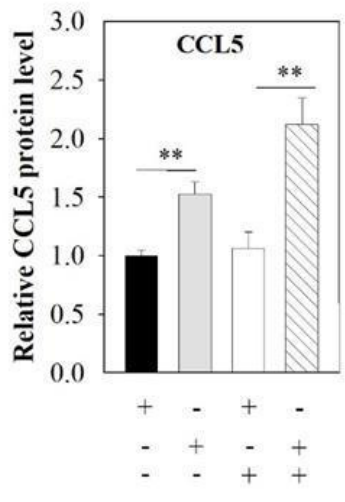

Figure 18. TNIP1 knockdown affects the downstream targets of the TLR signaling pathway. TNIP1 siRNA-mediated silencing (48 hours) was performed and HPV-KER cells were treated with C. acnes (MOI=100). (A) TNIP1 protein levels were analyzed by western blot and quantitated using the Image Pro Plus software. (Representative blot.) After 6 hours bacterial treatment, (B) NF- $\kappa$ B promoter activities were measured with a luciferase reporter assay, whereas (C) mRNA levels of TNF $\alpha$, IL-6, IL-8 and CCL5 were monitored by real-time RT-PCR. (D) After 24 hours bacterial treatment, secreted cytokine and chemokine levels were measured by ELISA and relative protein levels are presented. In luciferase reporter assay all data were normalized to Renilla, in western blot analyzes to actin, whereas in real-time RT-PCR analyzes to 18S rRNA and compared to the time-matched untreated samples transfected with scrambled siRNA (scr-siRNA). Statistical analysis: paired, two-sample t-test: $* \mathrm{p}<0.05, * * \mathrm{p}<0.01, * * * \mathrm{p}<0,001$. 
cDNA-based transient overexpression resulted in markedly increased TNIP1 protein levels in HPV-KER cells (Figure 17/A). As a consequence, significantly decreased constitutive and $C$. acnes-induced NF- $\mathrm{BB}$ promoter activities were measured in the subsequent luciferase reporter assay (Figure 17/B). mRNA expression of constitutive and $C$. acnes-induced TNF $\alpha$, IL-8 and CCL5 also decreased compared to the empty-vector transfected samples. Besides, bacterium-induced mRNA levels of IL-6 decreased, whereas IL-1 $\alpha$ levels were not affected (Figure 17/C). Overexpression of TNIP1 also decreased IL-6, IL-8 and CCL5 protein secretion (Figure 17/D).

In contrast, siRNA-mediated silencing of TNIP1 led to markedly decreased TNIP1 protein levels (Figure 18/A). As a consequence, significantly increased constitutive and bacterialinduced NF- $\mathrm{kB}$ promoter activities were found in a luciferase reporter assay (Figure 18/B). Constitutive and $C$. acnes-induced expression of TNFa, IL-8, and CCL5 mRNAs markedly increased, whereas IL-1 $\alpha$ and IL-6 expression increased moderately in response to TNIP1 silencing (Figure 18/C). Constitutive and bacterium-induced secretion of IL-8, IL-6, and CCL5 were also elevated in TNIP1 silenced HPV-KER cells (Figure 18/D).

\subsubsection{ATRA induces TNIP1 expression and affects the levels of downstream targets of the TLR signaling pathway}

The TNIP1 promoter contains retinoic acid response elements (RARE) and retinoic-acidreceptor binding sites. These elements are involved in the induction of TNIP1 expression by retinoic acid under permissive epigenetic conditions in different cell lines (60). Since ATRA is an effective drug used for acne treatment, we examined whether this compound is capable of regulating TNIP1 and, thus, the expression of downstream targets of the TLR signaling pathway in keratinocytes.

We observed that ATRA treatment led to slightly elevated TNIP1 mRNA levels, although this effect was not statistically significant (Figure 19/A), and to significantly increased TNIP1 protein expression (Figure 19/B). In addition, constitutive and C. acnes-induced mRNA expression of TLR-2 and the pro-inflammatory TNF $\alpha$ and CCL5 decreased upon ATRA treatment. In contrast, both constitutive and $C$. acnes-induced TLR-4 and IL-8 mRNA expression levels increased, whereas TLR3 and IL-6 mRNA levels were not affected by the drug (Figure 19/D, E). 
To confirm that the effects of ATRA on TNIP1 expression were not specific to the HPVKER cell line, we also applied the drug to the upper, epidermal part of OS models. We found that TNIP1 protein expression levels had increased in all epidermal layers 24 hours after ATRA treatment (Figure 19/C), in a manner similar to observed for immortalized keratinocytes.

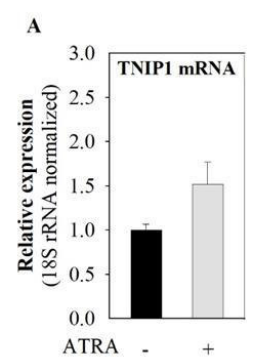

B
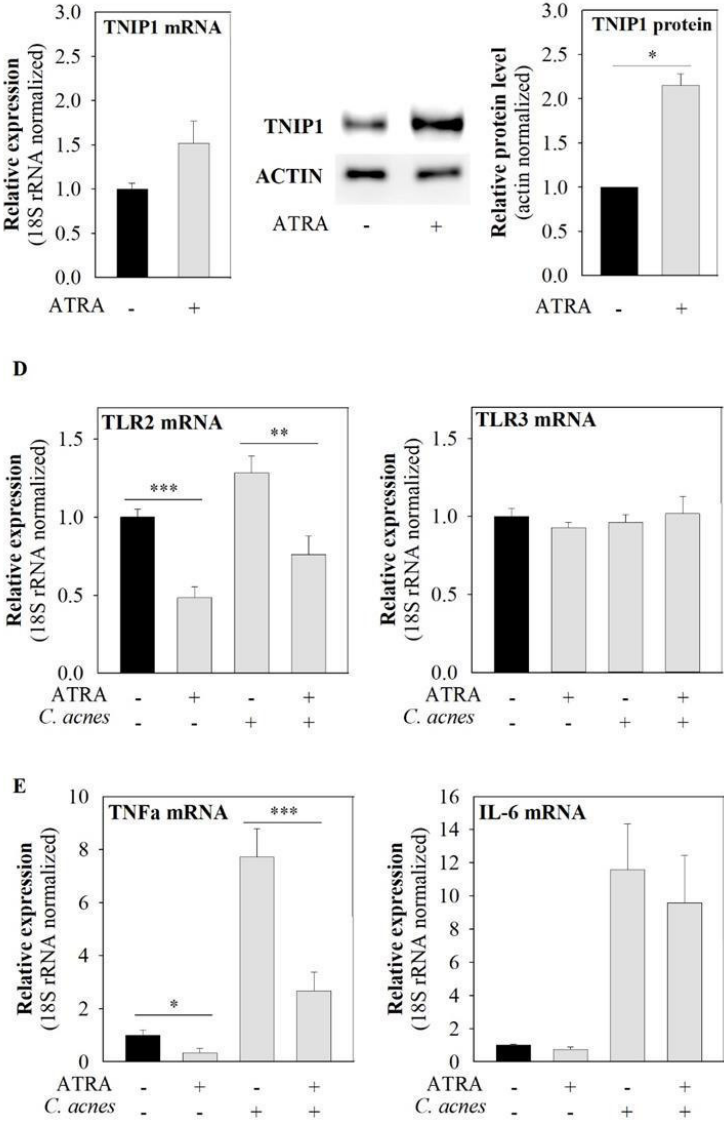
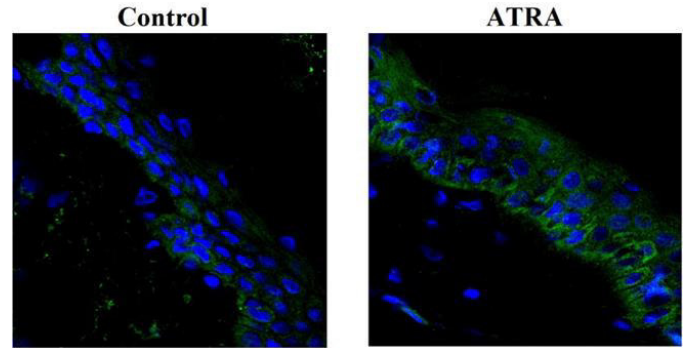

Figure 19. ATRA induces TNIP1 expression and affects the expression of TLRs and downstream targets of the TLR signaling pathways. HPV-KER cells (A, B) were treated with $10^{-6} \mathrm{M}$ ATRA for 48 hours or the OS cultures (C) with $1.5 \times 10^{-6} \mathrm{M}$ for 24 hours. TNIP1 mRNA levels were analyzed by real-time RTPCR, whereas protein levels were followed by western blot analyzes and quantitated using the Image Pro Plus software or visualized by immunofluorescence staining for TNIP1 (green) and DAPI (blue). Subsequently, HPVKER cells were challenged with $C$. acnes $(\mathrm{MOI}=100)$ for $6 \mathrm{~h}$ and the mRNA expression changes of TLRs (D) and selected pro-inflammatory molecules was analyzed by real-time RT-PCR. mRNA data were normalized to $18 \mathrm{~S}$ rRNA, protein levels were normalized to actin and compared to time-matched untreated samples. Errors are SD (B) or SE (A, D, E). Statistical analysis: paired, two-sample t-test: ${ }^{*} \mathrm{p}<0.05, * * \mathrm{p}<0.01,{ }^{* * *} \mathrm{p}<0.001$. 


\section{Discussion}

The human skin harbors a specialized microbiota that protects against pathogens, helps to maintain proper skin functions, and also serves as a part of the immunological barrier. These are not specific functions of the cutaneous microbial community, similar functions have also been identified for the gut microbiota (61). One important feature of the resident skin community is that its members keep the cutaneous immune system at a basal activated state. Such low levels of TLR activation may contribute to the maintenance of basal expression levels of different genes (e.g., cytokines, chemokines, and inducible antimicrobial peptides), playing important roles in the formation and maintenance of the immunological barrier (62). In healthy skin, these events do not lead to inflammation. However, under certain conditions, different commensal microbes may be involved in inflammatory skin diseases, such as $C$. acnes in acne pathogenesis $(30,63)$. The mechanisms underlying this duality - the protective and pathogenic aspects of the continual presence of the skin microbiota - are still unclear $(61,64)$.

C. acnes, a dominant member of the human skin microbiome, is recognized by TLR2 and TLR4 in epidermal keratinocytes. This bacterium can induce innate immune and inflammatory events, such as the production of inflammatory cytokines, chemokines, antimicrobial peptides, and other inflammatory mediators, as well as autophagy in keratinocytes, and, through this induction, plays a role in the pathogenesis of acne vulgaris $(21,22,63,65)$. However, at the beginning of early adolescence, this bacterium becomes overwhelmingly dominant in the skin, yet does not induce macroscopically detectable inflammation in the majority of the PSUs $(8,11)$. These observations suggest the existence of regulatory mechanisms that control $C$. acnes-induced immune activation.

In the past decades, several negative regulators of the TLR and the NF- $\mathrm{BB}$ signaling pathways have been identified. These play important roles in the protection against exaggerated immune activation. Since their discovery, an increasing number of publications have reported a correlation between genetic variations of these proteins and altered expression and/or dysfunctional proteins, as well as with diseases associated with inflammation, such as psoriasis, systemic lupus erythematosus, pneumonia and cancer $(34,66-69)$. Taken together, these results suggest that proper regulation and function of these factors are indispensable for 
the maintenance of health, but the role of these molecules in the assuagement of microbiomeinduced immune activation has been poorly investigated.

In our studies, we aimed to investigate whether well-known negative regulators of TLR signaling pathways, SIGIRR, TOLLIP, TNFAIP3 and TNIP1, have any role in the C. acnesinduced innate immune activation of keratinocytes. For that, we systematically analyzed their expression changes upon $C$. acnes challenge and their role in the bacterium-induced innate immune events using in vitro and ex vivo model systems.

SIGIRR is widely expressed in several cell types, among others, in cells that have direct contact with the human microbiota, including lung and intestinal epithelial cells (IECs) (70). In most cases, TLR stimulation by pathogens or synthetic TLR ligands dampens SIGIRR levels (40). We found that SIGIRR is also expressed in immortalized keratinocytes, but none of the $C$. acnes strains we used in our studies affected its expression in HPV-KER cells.

Many studies also showed, that decreased SIGIRR levels contributed to local and systemic inflammation upon pathogen infection in SIGIRR-deficient mice models, and enhanced inflammatory mediator production upon TLR ligand stimuli in SIGIRR-silenced intestinal epithelial cells $(40,71)$. In contrast, SIGIRR did not suppress TLR signaling in intrarenal and tubular epithelial cells, suggesting a cell-type specific role of this molecule $(72,73)$. In our experiments, transient, siRNA-mediated silencing of SIGIRR did not affect basal and $C$. acnes-induced TNF $\alpha$ mRNA expression. These results suggest, that even though SIGIRR is expressed in HPV-KER cells, it does not seem to play important roles in the control of $C$. acnes-induced signaling cascades in keratinocytes.

TOLLIP is also present in different microbiota-colonized surfaces, and elevated expression levels were found in response to different TLR ligands in IECs $(74,75)$. We found that TOLLIP is expressed in human, in vitro cultured keratinocytes, but its mRNA and protein levels remained unchanged upon $C$. acnes challenge.

Altered TOLLIP levels can influence the outcome of TLR2 and TLR4 activation, and inflammatory mediator production in IECs (75), and TOLLIP deficient mice were susceptible to colitis $(76,77)$. We experimentally modified TOLLIP levels by siRNA-mediated silencing and found that in the silenced cells, the basal and C. acnes-induced TNF $\alpha$ levels remained unchanged. These results suggest that TOLLIP is not a major regulator of the bacteriuminduced immune activation in keratinocytes. 
We also investigated the role of TNFAIP3 in the $C$. acnes-induced immune processes. In agreement with other studies (48), we showed that TNFAIP3 is expressed through the entire epidermis, exhibiting higher levels in the more differentiated keratinocyte layers. This gradient may be formed parallel with the natural differentiation processes, as one of the major regulators of keratinocyte differentiation is calcium $\left(\mathrm{Ca}^{2+}\right)$, and together with $\mathrm{Ca}^{2+}$-induced differentiation, TNFAIP3 expression also increases in normal human in vitro cultured keratinocytes $(48,78)$. The molecular details of these events are currently not known, but it may be mediated through the DREAM transcription factor, which is $\mathrm{Ca}^{2+}$ regulated and also plays important roles in the regulation of TNFAIP3 transcription $(58,79)$.

We also analyzed whether $C$. acnes influenced TNFAIP3 expression levels in vitro and ex vivo. We found that both the mRNA and protein expression rapidly increased in a dosedependent manner in response to the bacterium in cultured HPV-KER cells, as well as in the epidermal part of an ex vivo skin model. A similar effect was described recently for another member of the skin microbiota, S. epidermidis (49). These results, taken together, indicate that the TNFAIP3 protein may play important roles in the regulation of immune and inflammation activation in response to various members of the cutaneous microbiota.

According to our current knowledge, $C$. acnes plays an important role in the pathogenesis of acne vulgaris, as a result of microbial dysbiosis (11). We were curious to see whether TNFAIP3 levels differ in control individuals and in the skin samples from acne patients. To answer this question, we compared publicly available microarray data in the GEO Profile database (ID: 33444972) and found that in patients, mRNA expression of TNFAIP3 significantly increases in papules compared to the non-lesional skin samples of the same individuals. In contrast to that, no major differences were noted comparing the healthy controls and the normal-looking skin of patients. These results suggest that, in the lesional skin, apart from the production of different pro-inflammatory mediators, the expression of anti-inflammatory molecules also increases. These latter molecules may play important roles in inflammation control to avoid excessive, potentially tissue-damaging reactions. To determine whether increased TNFAIP3 mRNA levels in the lesional skin corresponds to elevated protein levels, further research is necessary. If mRNA and protein levels correspond, the accumulation may be an important step during the healing of lesions and the restoration of epidermal homeostasis. 
We also analyzed whether TNFAIP3 expression differs in response to different $C$. acnes strains. We found no major differences in the TNFAIP3 mRNA expression when the HPV-KER cells were co-cultured with C. acnes 889, ATCC11828 and 6609 bacteria strain.

Multiple signaling pathways and transcriptional factors are involved in the transcriptional regulation of TNFAIP3. In LPS-activated bone-marrow-derived macrophages from mice, NF- $\kappa \mathrm{B}$ and p38 regulate TNFAIP3 expression through its NF- $\kappa \mathrm{B}$ and C/EBPB binding sites (59). However, in Pam3CSK4-activated THP1 cells, NF-кB inhibition has no effect: GSK3related pathways regulate changes in TNFAIP3 expression, possibly through the cAMP response element-binding protein (CREB) (80). Others also showed that the DREAM complex represses its transcriptional activation through downstream-responsive elements (DRE3 and DRE4) binding sites in mice and HLMVECs (58). These findings suggest that the transcriptional regulation of TNFAIP3 is possibly dependent on the type of cell and stimuli. We found that the basal mRNA expression of TNFAIP3 is regulated by JNK, whereas JNK and NF- $\mathrm{KB}$ signaling pathways are involved in the control of $C$. acnes-induced changes in TNFAIP3 mRNA and protein expression, for which p38 and ERK1/2 inhibition had no effect. Dual inhibition of JNK and NF-אB signaling pathways almost completely inhibited C. acnesinduced changes in mRNA expression, which suggest that these two signaling pathways have a major impact on the bacterium-induced regulation of TNFAIP3 mRNA levels in HPV-KER cells.

Based on our findings, TNFAIP3 possibly operates in a negative regulatory cycle in keratinocytes. In this control loop, TNFAIP3 regulates the signaling consequences of $C$. acnes, which, in turn, also affects TNFAIP3 levels (Figure 20).

TNFAIP3 may help S. epidermidis to persist as a commensal on the human skin, through the control of bacterium-induced $\mathrm{NF}-\kappa \mathrm{B}$ activation, IL-1 $\beta$ and $\mathrm{hBD} 2$ production in keratinocytes (49). Based on these findings, we aimed to analyze whether this negative regulatory function also occurs in $C$. acnes-induced inflammatory processes. For these experiments, we found that silencing TNFAIP3 significantly increased basal NF- $\kappa$ B promoter activities, as well as the mRNA expression of pro-inflammatory cytokines and chemokines (TNF $\alpha$, IL-1 $\alpha$, IL-6, IL-8 and CCL5) in HPV-KER cells. Co-culturing of silenced cells with the $C$. acnes 889 strain also lead to increased TNF $\alpha$, IL-1 $\alpha$, IL-6, IL-8 and CCL5 mRNA expression, as well as elevated IL-8, IL-6 and CCL5 protein secretions. These data argue that 
TNFAIP3 plays important roles in the regulation of $C$. acnes-driven molecular events in keratinocytes as well as in the maintenance of cellular homeostasis by regulating the cutaneous inflammatory program under homeostatic conditions (81).

Apart from the control of the microbiota-induced processes, studies suggests that TNFAIP3 functions in, for example, poly-I:C-induced inflammatory responses in human keratinocytes, as well as in imiquimod- and acetone-induced psoriasis (PSO) and dermatitis in mouse models, respectively $(47,48)$. TNFAIP3 also plays important roles in the induction of Pam3CSK4-tolerance in monocytic THP-1 cells, arguing for a generalized role for this molecule in different TLR-mediated molecular processes (80).

Based on the analysis of publicly available data, we could show that TNFAIP3 levels differ in the non-lesional and lesional skin samples of acne patients. Interestingly, acne is not the only inflammatory skin disease in which TNFAIP3 levels change. In psoriasis and atopic dermatitis (AD), studies showed lower TNFAIP3 mRNA levels in lesional skin samples, compared to the skin from healthy individuals. Moreover, in PSO patients, TNFAIP3 mRNA and protein levels were lower in lesional skin than in non-lesional samples of the same patients. In contrast, TNFAIP3 mRNA expression levels in AD patients did not differ in the involved and non-involved samples $(47,48)$. It is not clear whether this observation represents a true difference in the pathogenesis of these chronic inflammatory skin conditions. One possible explanation is that the levels of negative regulators may rapidly change throughout the different phases of disease pathogenesis, and the above observations represent only temporal variations. It is interesting to note, however, that inability to control innate immune activation due to decreased TNFAIP3 levels may lead to persisting, chronic inflammation, and the net outcome and the severity of inflammation possibly depends on the balance of proand anti-inflammatory factors. As a result, this molecule may serve in the future as a drug target for treatment.

Last, but not least we also investigated the role of TNIP1, and we found that it is also a possible negative regulator of $C$. acnes-induced inflammatory events in HPV-KER cells. It is expressed in cultured keratinocytes, and its mRNA and protein expression increased in response to $C$. acnes treatment; and the extent of activation depended on the bacterial dose. These changes were not specific for the HPV-KER cell line, as similar results were obtained using NHEK cells. In ex vivo OS models we found that TNIP1 was expressed in all epidermal 
layers, which was consistent with previous studies (52). Some differences were also noted, as we detected mostly cytoplasmic and perinuclear staining within the keratinocytes, in contrast to previous studies, where cytoplasmic and also intensive nuclear staining of TNIP1 was observed in HaCaT cells $(52,82)$. The reason for these differences is not clear but may be caused by the differences in the properties of the used models, or the dissimilarities of the applied experimental conditions. When we analyzed the effect of the $C$. acnes treatment, we found elevated TNIP1 levels in the epidermis of the OS cultures. These results are in agreement with our findings using NHEK and HPV-KER cells, showing that, in addition to pro-inflammatory mediators, the bacterium also induces the expression of anti-inflammatory factors, possibly to avoid excessive inflammation and immune activation. Our results strongly suggest that TNIP1 may be one of the negative regulators of $C$. acnes-induced molecular events. These effects were independent of the used $C$. acnes strains, we did not observe any differences when we co-cultured our models with phylogenetically different strains. This was similar to what we observed in the case of TNFAIP3. These findings suggest that strainspecific biological effects of $C$. acnes are likely not due to the ability of differential induction of various negative regulators upon immune and inflammation induction in keratinocytes.

Next, were interested in the signaling pathways and regulatory molecules that may regulate TNIP1 levels under basal conditions and upon C. acnes induction. In silico analyzes predicted the presence of putative binding sites for NF- $\kappa$ B, AP-1, SP1, SP3 and C/EBPB in the TNIP1 promoter region $(59,83)$. Gene expression and ChIP assays have confirmed that NF- $\kappa \mathrm{B}$ and SP1 binding sites are active in HeLa cells $(82,84)$. Our finding that specific JNK, NF- $\kappa$ B, p38 and ERK1/2 inhibitors decreased the bacterium-induced upregulation of TNIP1 suggest that these signaling pathways may be involved in the regulation of $C$. acnes-induced TNIP1 expression in keratinocytes. Moreover, the intricate TNIP1 regulation pattern also suggests that this molecule may have important roles in different processes.

To confirm the negative regulatory role of TNIP1 in C. acnes-induced molecular events, we modified its endogenous levels by cDNA-based overexpression and siRNA-mediated silencing and examined the expression of downstream elements of TLR signaling pathways. $\mathrm{NF}-\mathrm{kB}$, one of the main mediators of signaling cascades activated in our experiments, is induced upon exposure to bacteria $(21,27)$. Our results, that TNIP1 overexpression decreased basal and $C$. acnes-induced NF-kB promoter activities and mRNA levels of TNF $\alpha$, IL-8, and 
CCL5 as well as secretion of IL-8, IL-6 and CCL5 correlated well with findings on other cell types. In HeLa cells, TNIP1 overexpression inhibited constitutive TNF $\alpha$, IL-1 $\alpha$ expression and lipopolysaccharide (LPS)-induced activation of NF- $\kappa \mathrm{B}(50,85)$.

In TNIP1-silenced cells, we observed increased constitutive NF- $\kappa \mathrm{B}$ promoter activities and elevated pro-inflammatory cytokine and chemokine mRNA and protein levels. TNIP1

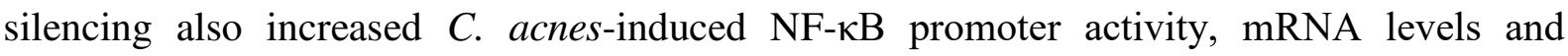
secretion of the mediators mentioned above. These results further support the conclusion that TNIP1 plays a role in the regulation of $C$. acnes-induced events in HPV-KER cells and possibly in the maintenance of homeostatic conditions. In a recent report, the authors have also demonstrated that TNIP1-silenced HaCaT cells were hypersensitive to synthetic TLR3 and TLR2/6 ligands and subsequently increased JNK and p38 phosphorylation and nuclear translocation of NF- $\kappa$ B. They also observed increased levels of secreted IL-6 and IL-8 compared to control cells (53). Overall, these data indicate a negative regulatory role of TNIP1 in TLR signaling events in keratinocytes.

Other reports have also shown the importance of TNIP1 as a gatekeeper in NF- $\mathrm{B}$, JNK, and p38 mediated processes, including the prevention of fetal liver apoptosis in a murine model and TNF $\alpha$-induced apoptosis in different cell lines $(86,87)$. In contrast, although TNIP1-deficient mice develop a progressive, lupus-like inflammatory disease, isolated TNIP1-deficient macrophages and dendritic cells showing no differences in pro-inflammatory signaling pathways with respect to $\mathrm{I} \kappa \mathrm{B} \alpha$ degradation and resynthesis and phosphorylation of different MAPKs (p38, ERK1/2, and JNK1/2) compared to wild type mice upon CpG-DNA or TLR4 (LPS) stimulation $(88,89)$. These findings suggest that the role of TNIP1 during TLR activation might be cell-type specific and depends on the nature of the stimuli.

Earlier studies found functional RARE elements in the TNIP1 promoter region, and that TNIP1 was induced by ATRA in HeLa cells under permissive epigenetic conditions, in the presence of Trichostatin A co-treatment (60). Trichostatin A inhibits histone deacetylase I and II and alters gene expression by opening chromatin and allowing transcription factors to bind, thus, promoting transcription of different genes. We applied ATRA, an active form of retinoic acid, to HPV-KER cells and found that TNIP1 mRNA and protein expression levels increased in response to the treatment without the addition of chromatin-modification agents. Furthermore, treatment of OS models led to similar results throughout the entire epidermis, 
suggesting that these effects were not specific to HPV-KER cells. It is not currently clear why HeLa cells behaved differently; however, differences in the duration of ATRA exposure or in the responsiveness of the different cells used might be responsible.

Despite the fact that retinoids, including ATRA, are widely used as an effective drug for acne therapy, the exact mechanism of action is not completely understood. Retinoids have been shown to promote cell proliferation, inhibit keratinocyte terminal differentiation, decrease the size of sebaceous glands and, indirectly, reduce the amount of $C$. acnes (90). Studies of the effect of retinoids on innate immunity are limited, and the results are often dependent on the cells used (60,91-94). In most cases, ATRA application to monocytes and macrophages decreased TLR2 abundance as well as the expression of selected proinflammatory mediators; however, the mechanism of these effects remains unclear $(93,95,96)$.

In our experiments, ATRA treatment decreased the level of constitutive and C. acnesinduced pro-inflammatory mediators TNF $\alpha$ and CCL5 but increased IL-8 levels. These results are consistent with the findings of others; similar IL-8 expression changes were observed in NHEK and also in other cell types in response to ATRA $(91,92,97)$. In NHEK cells, NF-кB and p38 signaling might contribute to these events (91).

Based on all these data, we conclude that ATRA may regulate TNIP1 expression and, as a consequence, negatively affect $C$. acnes-induced inflammatory events in keratinocytes. Our proposed model may offer a possible, novel mode of retinoid action in acne treatment.

TLR2 expression is increased in the epidermis and monocytes isolated from acne patients compared to healthy controls, and this increase might be a result of $C$. acnes-induced inflammatory events. ATRA treatment decreased TLR2 levels in monocytes isolated from both healthy donors and acne patients following isotretinoin therapy, although TLR4 expression was not affected $(21,95,96,98,99)$. We found similar changes in expression for TLR2 (decreased) and TLR4 (increased) in HPV-KER cells, suggesting that ATRA has opposite effects on these two receptors in keratinocytes.

We propose that the increased bacterial load in acne-prone follicles is a factor leading to the formation of inflammatory symptoms. Retinoids may attenuate $C$. acnes-induced inflammation by decreasing the sizes of sebaceous glands and sebum secretion and, subsequently, control the $C$. acnes load. In addition, TLR2 levels are also attenuated, preventing the sensing of this Gram-positive bacterium, which leads to deleterious 
inflammation. By decreasing the expression of TLR2, an important gatekeeper of the skin, the risk of opportunistic infections is increased. To modulate this effect, expression of another gatekeeper, TLR4 is elevated. Thus, multiple levels of sensing and signal transduction may be available in the defense against potentially harmful microbial invaders. The fact that opportunistic bacterial and fungal infections are rare in the lesional skin of acne patients is consistent with our hypothesis.

TNIP1 may regulate $C$. acnes-induced signaling events through the establishment of a negative-regulatory feedback loop controlling NF- $\mathrm{B}$ activity in keratinocytes, similarly to TNFAIP3. Other signaling pathways, such as nuclear-receptor signaling cascades that are activated by retinoids, may also affect TNIP1 levels, which in turn can modify the outcome of the induced processes (Figure 20).

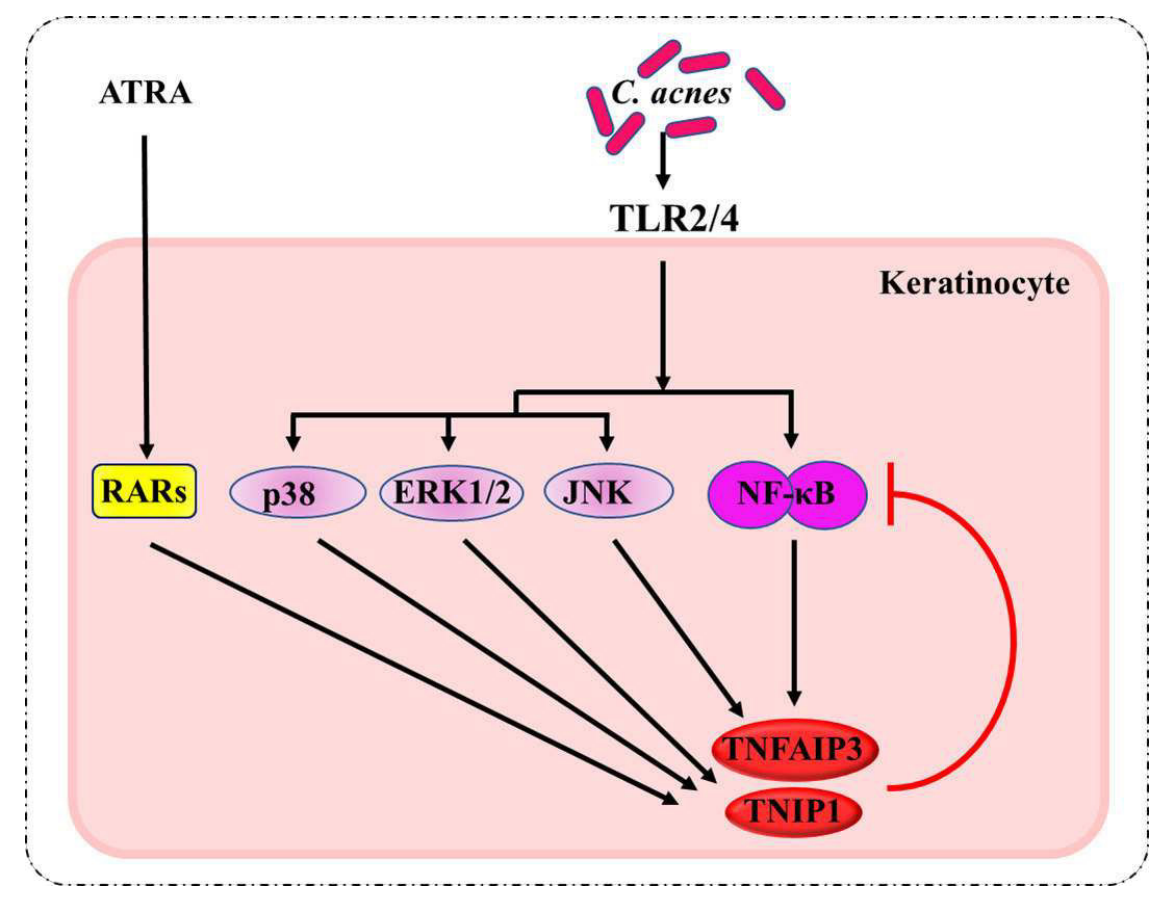

Figure 20. Negative regulation of $C$. acnes-induced immune activation in keratinocytes by TNFAIP3 and TNIP1.

In summary, TNFAIP3 and TNIP1 may function as negative regulators in keratinocytes that control $C$. acnes-induced inflammatory events, playing important roles in maintaining the homeostasis between the skin cells and the cutaneous microbiome. Development of novel, well-tolerated, TNFAIP3 and TNIP1-specific acne therapeutic modalities could potentially reduce inflammation without the harmful side effects of currently available treatment options (antibiotic, retinoid or hormonal formulation usage). 


\section{Summary}

Human skin cells recognize the presence of the skin microbiome through pathogen recognition receptors. Epidermal keratinocytes are known to activate TLR2 and TLR4 response to the commensal $C$. acnes bacterium, and subsequently induce innate immune and inflammatory events, which contributes to the pathogenesis of acne vulgaris. Healthy skin does not exhibit inflammation or skin lesions, even in the continuous presence of the same microbes. As the molecular mechanism for this duality is still unclear, we aimed to identify factors and mechanisms that control the innate immune response to $C$. acnes in keratinocytes.

We found that the expression of the well-known negative regulators of the TLR signaling cascade, TNIP1 and TNFAIP3, were rapidly induced in response to $C$. acnes in human, in vitro cultured keratinocytes as well as in OS models. Expression changes were dependent on the applied $C$. acnes dose, but not on the used bacterium strain. Bacterial-induced changes in TNIP1 expression were regulated by signaling pathways involving NF- $\kappa B$, p38, ERK1/2, and JNK, whereas TNFAIP3 expression were regulated by JNK and NF- $\kappa B$ pathways. Experimental modification of TNIP1 and TNFAIP3 levels affected the activity of NF- $\mathrm{BB}$ transcription factor and subsequent inflammatory cytokine and chemokine mRNA and protein levels. We found that all-trans retinoic acid (ATRA) induced elevated TNIP1 expression in HPV-KER cells and also in OS models, where TNIP1 levels increased throughout the epidermis. Based on these findings, we propose that ATRA may exhibit dual effects in acne therapy by both affecting the expression of the negative regulator TNIP1, and attenuating TLR2-induced inflammation.

In summary, TNFAIP3 and TNIP1 play important roles in the regulation of TLR-induced signaling pathways in keratinocytes, and, through these roles, contribute to the control of $C$. acnes-induced innate immune and inflammatory events (Figure 19). By fine-tuning the microbiota-induced signaling processes, these two molecules may help to maintain the homeostatic conditions between the skin and the cutaneous microflora. 


\section{Acknowledgement}

I would like to express my deep sense of gratitude to my supervisor, Dr. Kornélia Szabó, who supported and encouraged me during my scientific work. I am thankful for her scientific guidance, expertise, advices and patience.

I am also grateful to Prof. Dr. Lajos Kemény for providing me such a great opportunity to complete my $\mathrm{PhD}$ work in the Department. I express my thanks for his support and guidance.

I would like to thank to our collaborating partners, Dr. Edit Urbán and Prof. Dr. Katalin Burián for culturing and providing us $C$. acnes strains which were used in our experiments.

I am grateful to my colleagues, Beáta Szilvia Bolla, Dr. Renáta Bozó, Dr. Gábor Tax, Dr. Judit Danis and Dr. Anikó Göblös for their support, scientific advices, help during the experiments and for creating such a good atmosphere in the laboratory.

I wish to thank Andrea Tanácsné Bajkán for her excellent technical assistance, Viharosné Dósa-Rácz Éva for helping me in the statistical analysis and Lili Földi for the help during the preparation of the thesis.

I am indebted to my family and friends for their encouragement, patience, unconditional support and love throughout my life.

This work was supported by the GINOP-2.3.2-15-2016-00015 and OTKA NK105369 research grants, by the ÚNKP-18-3 of New National Excellence Program of the Ministry of Human Capacities and the ÚNKP-19-3 of New National Excellence Program of the Ministry for Innovation and Technology. The project has received funding from the EU's Horizon 2020 research and innovation program under grant agreement No. 739593, in the framework of the HCEMM-SZTE Skin Research Group. 


\section{References}

1. Kanitakis J. Anatomy, histology and immunohistochemistry of normal human skin. Eur J Dermatology (2002) 12:390-401.

2. Bressler RS, Bressler CH. Functional anatomy of the skin. Clin Podiatr Med Surg (1989) 6:229-246. doi:10.2174/9781681086910118010006

3. Grice EA, Kong HH, Conlan S, Deming CB, Davis J, Young AC, Bouffard GG, Blakesley RW, Murray PR, Green ED, et al. Topographical and temporal diversity of the human skin microbiome. Science (80- ) (2009) 324:1190-1192. doi:10.1126/science. 1171700

4. Jahns AC, Alexeyev OA. Three dimensional distribution of Propionibacterium acnes biofilms in human skin. Exp Dermatol (2014) 23:687-689. doi:10.1111/exd.12482

5. Grice EA, Segre JA. The skin microbiome. Nat Rev Microbiol (2011) 9:244-253. doi:10.1038/nrmicro2537

6. Oh J, Byrd AL, Deming C, Conlan S, Kong HH, Segre JA, Barnabas B, Blakesley R, Bouffard G, Brooks S, et al. Biogeography and individuality shape function in the human skin metagenome. Nature (2014) 514:59-64. doi:10.1038/nature13786

7. Oh J, Conlan S, Polley E, Segre J a, Kong HH. Shifts in human skin and nares microbiota of healthy children and adults. Genome Med (2012) 4:77. doi:10.1186/gm378

8. Oh J, Conlan S, Polley EC, Segre JA, Kong HH. Shifts in human skin and nares microbiota of healthy children and adults. Genome Med (2012) 4:77. doi:10.1186/gm378

9. Kong HH, Segre JA. The Molecular Revolution in Cutaneous Biology: Investigating the Skin Microbiome. J Invest Dermatol (2017) 137:e119-e122. doi:10.1016/j.jid.2016.07.045

10. Grice EA, Kong HH, Renaud G, Young AC, Bouffard GG, Blakesley RW, Wolfsberg TG, Turner ML, Segre JA. A diversity profile of the human skin microbiota. Genome Res (2008) 18:1043-1050. doi:10.1101/gr.075549.107

11. Szabó K, Erdei L, Bolla BS, Tax G, Bíró T, Kemény L. Factors shaping the composition of the cutaneous microbiota. Br J Dermatol (2017) 176:344-351. 
doi:10.1111/bjd.14967

12. Sanford JA, Gallo RL. Functions of the skin microbiota in health and disease. Semin Immunol (2013) 25:370-377. doi:10.1016/j.smim.2013.09.005

13. Shu M, Wang Y, Yu J, Kuo S, Coda A, Jiang Y, Gallo RL, Huang CM. Fermentation of Propionibacterium acnes, a Commensal Bacterium in the Human Skin Microbiome, as Skin Probiotics against Methicillin-Resistant Staphylococcus aureus. PLoS One (2013) 8: doi:10.1371/journal.pone.0055380

14. Christensen GJM, Brüggemann H. Bacterial skin commensals and their role as host guardians. Benef Microbes (2014) 5:201-215. doi:10.3920/BM2012.0062

15. Erin Chen Y, Fischbach MA, Belkaid Y. Skin microbiota-host interactions. Nature (2018) 553:427-436. doi:10.1038/nature25177

16. Dréno B, Araviiskaia E, Berardesca E, Gontijo G, Sanchez Viera M, Xiang LF, Martin R, Bieber T. Microbiome in healthy skin, update for dermatologists. J Eur Acad Dermatology Venereol (2016) 30:2038-2047. doi:10.1111/jdv.13965

17. Findley K, Grice EA. The Skin Microbiome: A Focus on Pathogens and Their Association with Skin Disease. PLoS Pathog (2014) 10: doi:10.1371/journal.ppat.1004436

18. Pivarcsi A, Kemény L, Dobozy A. Innate Immune Functions of the Keratinocytes. Acta Microbiol Immunol Hung (2004) 51:303-310. doi:10.1556/AMicr.51.2004.3.8

19. Miller LS. Toll-Like Receptors in Skin. Adv Dermatol (2008) 24:71-87. doi:10.1016/j.yadr.2008.09.004

20. De Koning HD, Rodijk-Olthuis D, Van Vlijmen-Willems IMJJ, Joosten LAB, Netea MG, Schalkwijk J, Zeeuwen PLJM. A comprehensive analysis of pattern recognition receptors in normal and inflamed human epidermis: Upregulation of dectin-1 in psoriasis. J Invest Dermatol (2010) 130:2611-2620. doi:10.1038/jid.2010.196

21. Kim J, Ochoa M-T, Krutzik SR, Takeuchi O, Uematsu S, Legaspi AJ, Brightbill HD, Holland D, Cunliffe WJ, Akira S, et al. Activation of Toll-Like Receptor 2 in Acne Triggers Inflammatory Cytokine Responses. J Immunol (2002) 169:1535-1541. doi:10.4049/jimmunol.169.3.1535

22. Pivarcsi A. Expression and function of Toll-like receptors 2 and 4 in human keratinocytes. Int Immunol (2003) 15:721-730. doi:10.1093/intimm/dxg068 
23. Nguyen CT, Sah SK, Kim TY. Inhibitory effects of superoxide dismutase 3 on Propionibacterium acnes-induced skin inflammation. Sci Rep (2018) 8: doi:10.1038/s41598-018-22132-z

24. Wang YY, Ryu AR, Jin S, Jeon YM, Lee MY. Chlorin e6-mediated Photodynamic therapy suppresses P. acnes-induced inflammatory response via NFאB and MAPKs signaling pathway. PLoS One (2017) 12: doi:10.1371/journal.pone.0170599

25. Pretsch A, Nagl M, Schwendinger K, Kreiseder B, Wiederstein M, Pretsch D, Genov M, Hollaus R, Zinssmeister D, Debbab A, et al. Antimicrobial and anti-inflammatory activities of endophytic fungi Talaromyces wortmannii extracts against acne-inducing bacteria. PLoS One (2014) 9: doi:10.1371/journal.pone.0097929

26. Koreck A, Pivarcsi A, Dobozy A, Kemény L. The role of innate immunity in the pathogenesis of acne. Dermatology (2003) 206:96-105. doi:10.1159/000068476

27. Nagy I, Pivarcsi A, Koreck A, Széll M, Urbán E, Kemény L. Distinct strains of Propionibacterium acnes induce selective human $\beta$-defensin-2 and interleukin-8 expression in human keratinocytes through toll-like receptors. J Invest Dermatol (2005) 124:931-938. doi:10.1111/j.0022-202X.2005.23705.x

28. Lovászi M, Mattii M, Eyerich K, Gácsi A, Csányi E, Kovács D, Rühl R, Szegedi A, Kemény L, Ståhle M, et al. Sebum lipids influence macrophage polarization and activation. Br J Dermatol (2017) 177:1671-1682. doi:10.1111/bjd.15754

29. Mattii M, Lovászi M, Garzorz N, Atenhan A, Quaranta M, Lauffer F, Konstantinow A, Küpper M, Zouboulis CC, Kemeny L, et al. Sebocytes contribute to skin inflammation by promoting the differentiation of T helper 17 cells. Br J Dermatol (2018) 178:722730. doi:10.1111/bjd.15879

30. Dréno B. What is new in the pathophysiology of acne, an overview. J Eur Acad Dermatology Venereol (2017) 31:8-12. doi:10.1111/jdv.14374

31. Szabó K, Kemény L. Studying the genetic predisposing factors in the pathogenesis of acne vulgaris. Hum Immunol (2011) 72:766-773. doi:10.1016/j.humimm.2011.05.012

32. Grice EA, Segre JA. The skin microbiome. Nat Rev Microbiol (2011) 9:244-253. doi:10.1038/nrmicro2537

33. Szegedi A, Dajnoki Z, Bíró T, Kemény L, Törőcsik D. Acne: Transient Arrest in the Homeostatic Host-Microbiota Dialog? Trends Immunol (2019) 40:873-876. 
doi:10.1016/j.it.2019.08.006

34. Liew FY, Xu D, Brint EK, O’Neill LAJ. Negative regulation of toll-like receptormediated immune responses. Nat Rev Immunol (2005) 5:446-458. doi:10.1038/nri1630

35. Miggin SM, O’Neill L a J. New insights into the regulation of TLR signaling. J Leukoc Biol (2006) 80:220-226. doi:10.1189/jlb.1105672.0741-5400/06/0080-220

36. Kondo T, Kawai T, Akira S. Dissecting negative regulation of Toll-like receptor signaling. Trends Immunol (2012) 33:449-458. doi:10.1016/j.it.2012.05.002

37. Wang J, Hu Y, Deng WW, Sun B. Negative regulation of Toll-like receptor signaling pathway. Microbes Infect (2009) 11:321-327. doi:10.1016/j.micinf.2008.12.011

38. Kadota C, Ishihara S, Aziz MM, Rumi MA, Oshima N, Mishima Y, Moriyama I, Yuki T, Amano Y, Kinoshita Y. Down-regulation of single immunoglobulin interleukin-1Rrelated molecule (SIGIRR)/TIR8 expression in intestinal epithelial cells during inflammation. Clin Exp Immunol (2010) 162:348-361. doi:10.1111/j.13652249.2010.04254.x

39. Costello DA, Carney DG, Lynch MA. $\alpha$-TLR2 antibody attenuates the A $\beta$-mediated inflammatory response in microglia through enhanced expression of SIGIRR. Brain Behav Immun (2015) 46:70-79. doi:10.1016/j.bbi.2015.01.005

40. Molgora M, Supino D, Mantovani A, Garlanda C. Tuning inflammation and immunity by the negative regulators IL-1R2 and IL-1R8. Immunol Rev (2018) 281:233-247. doi:10.1111/imr.12609

41. Sham HP, Yu EYS, Gulen MF, Bhinder G, Stahl M, Chan JM, Brewster L, Morampudi V, Gibson DL, Hughes MR, et al. SIGIRR, a Negative Regulator of TLR/IL-1R Signalling Promotes Microbiota Dependent Resistance to Colonization by Enteric Bacterial Pathogens. PLoS Pathog (2013) 9: doi:10.1371/journal.ppat.1003539

42. Zhang G, Ghosh S. Negative regulation of toll-like receptor-mediated signaling by Tollip. J Biol Chem (2002) 277:7059-7065. doi:10.1074/jbc.M109537200

43. $\mathrm{Li} \mathrm{T,} \mathrm{Hu} \mathrm{J,} \mathrm{Li} \mathrm{L.} \mathrm{Characterization} \mathrm{of} \mathrm{Tollip} \mathrm{protein} \mathrm{upon} \mathrm{Lipopolysaccharide} \mathrm{challenge.}$ Mol Immunol (2004) doi:10.1016/j.molimm.2004.03.009

44. Kowalski EJA, Li L. Toll-interacting protein in resolving and non-resolving inflammation. Front Immunol (2017) 8: doi:10.3389/fimmu.2017.00511

45. Malynn BA, Ma A. A20: A multifunctional tool for regulating immunity and 
preventing disease. Cell Immunol (2019) 340:103914. doi:10.1016/j.cellimm.2019.04.002

46. Das T, Chen Z, Hendriks RW, Kool M. A20/tumor necrosis factor $\alpha$-induced protein 3 in immune cells controls development of autoinflammation and autoimmunity: Lessons from mouse models. Front Immunol (2018) 9: doi:10.3389/fimmu.2018.00104

47. Sohn KC, Back SJ, Choi DK, Shin JM, Kim SJ, Im M, Lee Y, Seo YJ, Yoon TJ, Lee $\mathrm{YH}$, et al. The inhibitory effect of A20 on the inflammatory reaction of epidermal keratinocytes. Int J Mol Med (2016) 37:1099-1104. doi:10.3892/ijmm.2016.2514

48. Devos M, Mogilenko DA, Fleury S, Gilbert B, Becquart C, Quemener S, Dehondt H, Tougaard P, Staels B, Bachert C, et al. Keratinocyte Expression of A20/TNFAIP3 Controls Skin Inflammation Associated with Atopic Dermatitis and Psoriasis. J Invest Dermatol (2019) 139:135-145. doi:10.1016/j.jid.2018.06.191

49. Simanski M, Erkens AS, Rademacher F, Harder J. Staphylococcus epidermidisinduced interleukin-1 beta and human beta-defensin-2 expression in human keratinocytes is regulated by the host molecule a20 (Tnfaip3). Acta Derm Venereol (2019) 99:181-187. doi:10.2340/00015555-3073

50. Mauro C, Pacifico F, Lavorgna A, Mellone S, Iannetti A, Acquaviva R, Formisano S, Vito P, Leonardi A. ABIN-1 binds to NEMO/IKK $\gamma$ and co-operates with A20 in inhibiting NF-кB. $J$ Biol Chem (2006) 281:18482-18488. doi:10.1074/jbc.M601502200

51. G'Sell RT, Gaffney PM, Powell DW. A20-binding inhibitor of NF-??B activation 1 is a physiologic inhibitor of NF-??B: A molecular switch for inflammation and autoimmunity. Arthritis Rheumatol (2015) 67:2292-2302. doi:10.1002/art.39245

52. Chen Y, Yan H, Song Z, Chen F, Wang H, Niu J, Shi X, Zhang D, Zhang N, Zhai Z, et al. Downregulation of TNIP1 expression leads to increased proliferation of human keratinocytes and severer psoriasis-like conditions in an imiquimod-induced mouse model of dermatitis. PLoS One (2015) 10:1-18. doi:10.1371/journal.pone.0127957

53. Rudraiah S, Shamilov R, Aneskievich BJ. TNIP1 reduction sensitizes keratinocytes to post-receptor signalling following exposure to TLR agonists. Cell Signal (2018) 45:8192. doi:10.1016/j.cellsig.2018.02.004

54. Mohan C, Zhu J. Toll-like receptor signaling pathways - Therapeutic opportunities. 
Mediators Inflamm (2010) 2010: doi:10.1155/2010/781235

55. Tax G, Urbán E, Palotás Z, Puskás R, Kónya Z, Bíró T, Kemény L, Szabó K. Propionic acid produced by Propionibacterium acnes strains contributes to their pathogenicity. Acta Derm Venereol (2016) 96:43-49. doi:10.2340/00015555-2154

56. Trivedi NR, Gilliland KL, Zhao W, Liu W, Thiboutot DM. Gene array expression profiling in acne lesions reveals marked upregulation of genes involved in inflammation and matrix remodeling. J Invest Dermatol (2006) 126:1071-1079. doi:10.1038/sj.jid.5700213

57. Lheure C, Grange PA, Ollagnier G, Morand P, Désiré N, Sayon S, Corvec S, Raingeaud J, Marcelin AG, Calvez V, et al. TLR-2 recognizes propionibacterium acnes CAMP factor 1 from highly inflammatory strains. PLoS One (2016) 11: doi:10.1371/journal.pone.0167237

58. Tiruppathi C, Soni D, Wang DM, Xue J, Singh V, Thippegowda PB, Cheppudira BP, Mishra RK, Debroy A, Qian Z, et al. The transcription factor DREAM represses the deubiquitinase A20 and mediates inflammation. Nat Immunol (2014) 15:239-247. doi:10.1038/ni.2823

59. Lai TY, Wu SD, Tsai MH, Chuang EY, Chuang LL, Hsu LC, Lai LC. Transcription of Tnfaip3 is regulated by $\mathrm{NF}-\kappa \mathrm{B}$ and $\mathrm{p} 38$ via $\mathrm{C} / \mathrm{EBP} \beta$ in activated macrophages. PLoS One (2013) 8: doi:10.1371/journal.pone.0073153

60. Gurevich I, Zhang C, Francis N, Struzynsky CP, Livings SE, Aneskievich BJ. Human $\mathrm{TNF} \alpha$-induced protein 3-interacting protein 1 (TNIP1) promoter activation is regulated by retinoic acid receptors. Gene (2013) 515:42-48. doi:10.1016/j.gene.2012.11.041

61. Chu H, Mazmanian SK. Innate immune recognition of the microbiota promotes hostmicrobial symbiosis. Nat Immunol (2013) 14:668-675. doi:10.1038/ni.2635

62. Kinnebrew MA, Pamer EG. Innate immune signaling in defense against intestinal microbes. Immunol Rev (2012) 245:113-131. doi:10.1111/j.1600-065X.2011.01081.x

63. Beylot C, Auffret N, Poli F, Claudel J-P, Leccia M-T, Del Giudice P, Dreno B. Propionibacterium acnes: an update on its role in the pathogenesis of acne. J Eur Acad Dermatol Venereol (2014) 28:271-8. doi:10.1111/jdv.12224

64. Gallo RL, Nakatsuji T. Microbial symbiosis with the innate immune defense system of the skin. J Invest Dermatol (2011) 131:1974-1980. doi:10.1038/jid.2011.182 
65. Megyeri K, Orosz L, Bolla S, Erdei L, Rázga Z, Seprényi G, Urbán E, Szabó K, Kemény L. Propionibacterium acnes Induces Autophagy in Keratinocytes: Involvement of Multiple Mechanisms. J Invest Dermatol (2018) 138:750-759. doi:10.1016/j.jid.2017.11.018

66. O'Rielly DD, Jani M, Rahman P, Elder JT. The Genetics of Psoriasis and Psoriatic Arthritis. J Rheumatol Suppl (2019) doi:10.3899/jrheum.190119

67. Zhu Y, Wang DG, Yang XK, Tao SS, Huang Q, Pan HF, Feng CC, Ye DQ. Emerging Role of SIGIRR rs7396562(T/G) Polymorphism in Systemic Lupus Erythematosus in a Chinese Population. Inflammation (2014) doi:10.1007/s10753-014-9916-z

68. Shah JA, Emery R, Lee B, Venkatasubramanian S, Simmons JD, Brown M, Hung CF, Prins JM, Verbon A, Hawn TR, et al. TOLLIP deficiency is associated with increased resistance to Legionella pneumophila pneumonia. Mucosal Immunol (2019) doi:10.1038/s41385-019-0196-7

69. Li C, Zhao Z, Zhou J, Liu Y, Wang H, Zhao X. Relationship between the TERT, TNIP1 and OBFC1 genetic polymorphisms and susceptibility to colorectal cancer in Chinese Han population. Oncotarget (2017) doi:10.18632/oncotarget.18378

70. Wald D, Qin J, Zhao Z, Qian Y, Naramura M, Tian L, Towne J, Sims JE, Stark GR, Li X. SIGIRR, a negative regulator of Toll-like receptor - Interleukin 1 receptor signaling. Nat Immunol (2003) doi:10.1038/ni968

71. Khan MA, Steiner TS, Sham HP, Bergstrom KS, Huang JT, Assi K, Salh B, Tai IT, Li X, Vallance BA. The Single IgG IL-1-Related Receptor Controls TLR Responses in Differentiated Human Intestinal Epithelial Cells. J Immunol (2010) doi:10.4049/jimmunol.0900021

72. Skuginna V, Lech M, Allam R, Ryu M, Clauss S, Susanti HE, Römmele C, Garlanda C, Mantovani A, Anders HJ. Toll-like receptor signaling and sigirr in renal fibrosis upon unilateral ureteral obstruction. PLoS One (2011) doi:10.1371/journal.pone.0019204

73. Lech M, Garlanda C, Mantovani A, Kirschning CJ, Schlöndorff D, Anders HJ. Different roles of TiR8/Sigirr on toll-like receptor signaling in intrarenal antigenpresenting cells and tubular epithelial cells. Kidney Int (2007) doi:10.1038/sj.ki.5002293

74. Sugi Y, Takahashi K, Nakano K, Hosono A, Kaminogawa S. Transcription of the 
Tollip gene is elevated in intestinal epithelial cells through impaired O-GlcNAcylationdependent nuclear translocation of the negative regulator Elf-1. Biochem Biophys Res Commun (2011) doi:10.1016/j.bbrc.2011.08.035

75. Otte JM, Cario E, Podolsky DK. Mechanisms of Cross Hyporesponsiveness to TollLike Receptor Bacterial Ligands in Intestinal Epithelial Cells. Gastroenterology (2004) doi:10.1053/j.gastro.2004.01.007

76. Begka C, Velin D, Maillard MH. Preventing Overheating: Tight Control of Gut Innate Immunity in Health and Disease. Inflamm Bowel Dis (2016) doi:10.1097/MIB.0000000000000838

77. Diao N, Zhang Y, Chen K, Yuan R, Lee C, Geng S, Kowalski E, Guo W, Xiong H, Li M, et al. Deficiency in Toll-interacting protein (Tollip) skews inflamed yet incompetent innate leukocytes in vivo during DSS-induced septic colitis. Sci Rep (2016) doi:10.1038/srep34672

78. Celli A, Sanchez S, Behne M, Hazlett T, Gratton E, Mauro T. The epidermal Ca2+ gradient: Measurement using the phasor representation of fluorescent lifetime imaging. Biophys J (2010) 98:911-921. doi:10.1016/j.bpj.2009.10.055

79. Sours-Brothers S, Ma R, Koulen P. Ca2+-sensitive transcriptional regulation: Direct DNA interaction by DREAM. Front Biosci (2009) 14:1851-1856. doi:10.2741/3346

80. Hu J, Wang G, Liu X, Zhou L, Jiang M, Yang L. A20 is critical for the induction of Pam3CSK4-tolerance in monocytic THP-1 cells. PLoS One (2014) 9: doi:10.1371/journal.pone.0087528

81. Lippens S, Lefebvre S, Gilbert B, Sze M, Devos M, Verhelst K, Vereecke L, Guire CM, Guérin C, Vandenabeele $\mathrm{P}$, et al. Keratinocyte-specific ablation of the NF- $\kappa \mathrm{B}$ regulatory protein A20 (TNFAIP3) reveals a role in the control of epidermal homeostasis. Cell Death Differ (2011) 18:1845-1853. doi:10.1038/cdd.2011.55

82. Gurevich I, Zhang C, Encarnacao PC, Struzynski CP, Livings SE, Aneskievich BJ. PPAR $\gamma$ and NF- $\kappa B$ regulate the gene promoter activity of their shared repressor, TNIP1. Biochim Biophys Acta - Gene Regul Mech (2012) 1819:1-15. doi:10.1016/j.bbagrm.2011.09.006

83. Verstrepen L, Carpentier I, Verhelst K, Beyaert R. ABINs: A20 binding inhibitors of NF- $\mathrm{BB}$ and apoptosis signaling. Biochem Pharmacol (2009) 78:105-114. 
doi:10.1016/j.bcp.2009.02.009

84. Encarnacao PC, Ramirez VP, Zhang C, Aneskievich BJ. Sp sites contribute to basal and inducible expression of the human TNIP1 (TNF $\alpha$-inducible protein 3-interacting protein 1) promoter. Biochem J (2013) 452:519-29. doi:10.1042/BJ20121666

85. Heyninck K, Valck D De, Berghe W Vanden, Criekinge W Van, Contreras R, Fiers W, Haegeman G, Beyaert R. Gene Expression by Interfering with an RIP- or TRAF2mediated Protein ABIN. Cell (1999) 145:1471-1482. doi:10.1083/jcb.145.7.1471

86. Oshima S, Turer EE, Callahan JA, Chai S, Advincula R, Barrera J, Shifrin N, Lee B, Yen B, Woo T, et al. ABIN-1 is a ubiquitin sensor that restricts cell death and sustains embryonic development. Nature (2009) 457:906-909. doi:10.1038/nature07575

87. Ramirez VP, Gurevich I, Aneskievich BJ. Emerging roles for TNIP1 in regulating post-receptor signaling. Cytokine Growth Factor Rev (2012) 23:109-118. doi:10.1016/j.cytogfr.2012.04.002

88. Nanda SK, Venigalla RKC, Ordureau A, Patterson-Kane JC, Powell DW, Toth R, C. Arthur JS, Cohen P. Polyubiquitin binding to ABIN1 is required to prevent autoimmunity. J Exp Med (2011) 208:1215-1228. doi:10.1084/jem.20102177

89. Zhou J, Wu R, High AA, Slaughter CA, Finkelstein D, Rehg JE, Redecke V, Hacker H. A20-binding inhibitor of NF-B (ABIN1) controls Toll-like receptor-mediated CCAAT/enhancer-binding protein activation and protects from inflammatory disease. Proc Natl Acad Sci (2011) 108:E998-E1006. doi:10.1073/pnas.1106232108

90. Ganceviciene R, Zouboulis CC. Isotretinoin: state of the art treatment for acne vulgaris. $J$ Dtsch Dermatol Ges (2010) 8 Suppl 1:S47-S59. doi:10.1111/j.16100387.2009.07238.x

91. Dai X, Yamasaki K, Shirakata Y, Sayama K, Hashimoto K. All-trans-retinoic acid induces interleukin-8 via the nuclear factor-kappaB and p38 mitogen-activated protein kinase pathways in normal human keratinocytes. J Invest Dermatol (2004) 123:10781085. doi:10.1111/j.0022-202X.2004.23503.X

92. Wojtal KA, Wolfram L, Frey-Wagner I, Lang S, Scharl M, Vavricka SR, Rogler G. The effects of vitamin A on cells of innate immunity in vitro. Toxicol Vitr (2013) 27:1525-1532. doi:10.1016/j.tiv.2013.03.013

93. Kim SY, Koo JE, Song MR, Lee JY. Retinol suppresses the activation of toll-like 
receptors in MyD88- and STAT1-independent manners. Inflammation (2013) 36:426433. doi:10.1007/s10753-012-9562-2

94. Kelhälä HL, Fyhrquist N, Palatsi R, Lehtimäki S, Väyrynen JP, Kubin ME, Kallioinen M, Alenius H, Tasanen K, Lauerma A. Isotretinoin treatment reduces acne lesions but not directly lesional acne inflammation. Exp Dermatol (2016) 25:477-478. doi:10.1111/exd.12971

95. Liu PT, Krutzik SR, Kim J, Modlin RL. Cutting Edge: All-trans Retinoic Acid DownRegulates TLR2 Expression and Function. J Immunol (2005) 174:2467-2470. doi:10.4049/jimmunol.174.5.2467

96. Dispenza MC, Wolpert EB, Gilliland KL, Dai JP, Cong Z, Nelson AM, Thiboutot DM. Systemic isotretinoin therapy normalizes exaggerated TLR-2-mediated innate immune responses in acne patients. J Invest Dermatol (2012) 132:2198-2205. doi:10.1038/jid.2012.111

97. Harant H, De Martin R, Andrew PJ, Foglar E, Dittrich C, Lindley IJ. Synergistic activation of interleukin-8 gene transcription by all-trans-retinoic acid and tumor necrosis factor-alpha involves the transcription factor NF-kappaB. J Biol Chem (1996)

98. Jugeau S, Tenaud I, Knol AC, Jarrousse V, Quereux G, Khammari A, Dreno B. Induction of toll-like receptors by Propionibacterium acnes. Br J Dermatol (2005) 153:1105-1113. doi:10.1111/j.1365-2133.2005.06933.X

99. Fathy A, Mohamed RW, Ismael NA, El-Akhras MA. Expression of toll-like receptor 2 on peripheral blood monocytes of patients with inflammatory and noninflammatory acne vulgaris. Egypt J Immunol (2009) 16:127-134. 


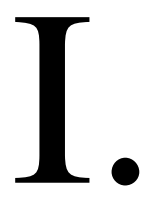




\section{OPEN ACCESS}

Edited by:

Thomas A. Kufer,

University of Hohenheim, Germany

Reviewed by:

Birgit Schittek

Universitäts-Hautklinik Tübingen,

Germany

Sylvie Legrand-Poels,

Université de Liège, Belgium

*Correspondence:

Lilla Erdei

erdei.lilla@med.u-szeged.hu

Kornélia Szabó

szabo.kornelia@med.u-szeged.hu

Specialty section

This article was submitted to

Molecular Innate Immunity,

a section of the journal

Frontiers in Immunology

Received: 07 March 2018

Accepted: 31 August 2018

Published: 24 September 2018

Citation:

Erdei L, Bolla BS, Bozó R, Tax G,

Urbán E, Kemény L and Szabó K

(2018) TNIP1 Regulates Cutibacterium

acnes-Induced Innate Immune

Functions in Epidermal Keratinocytes.

Front. Immunol. 9:2155.

doi: 10.3389/fimmu.2018.02155

\section{TNIP1 Regulates Cutibacterium acnes-Induced Innate Immune Functions in Epidermal Keratinocytes}

\author{
Lilla Erdei ${ }^{1 *}$, Beáta Szilvia Bolla ${ }^{1}$, Renáta Bozó ${ }^{1}$, Gábor Tax ${ }^{1}$, Edit Urbán ${ }^{2}$, \\ Lajos Kemény ${ }^{1,3}$ and Kornélia Szabó ${ }^{3 *}$ \\ ${ }^{1}$ Department of Dermatology and Allergology, University of Szeged, Szeged, Hungary, ${ }^{2}$ Institute of Clinical Microbiology, \\ University of Szeged, Szeged, Hungary, ${ }^{3}$ MTA-SZTE Dermatological Research Group, Szeged, Hungary
}

Human skin cells recognize the presence of the skin microbiome through pathogen recognition receptors. Epidermal keratinocytes are known to activate toll-like receptors (TLRs) 2 and 4 in response to the commensal Cutibacterium acnes (C. acnes, formerly known as Propionibacterium acnes) bacterium and subsequently to induce innate immune and inflammatory events. These events may lead to the appearance of macroscopic inflammatory acne lesions in puberty: comedos, papules and, pustules. Healthy skin does not exhibit inflammation or skin lesions, even in the continuous presence of the same microbes. As the molecular mechanism for this duality is still unclear, we aimed to identify factors and mechanisms that control the innate immune response to $C$. acnes in keratinocytes using a human immortalized keratinocyte cell line, HPV-KER, normal human keratinocytes (NHEK) and an organotypic skin model (OSM). TNIP1, a negative regulator of the NF-kB signaling pathway, was found to be expressed in HPV-KER cells, and its expression was rapidly induced in response to $C$. acnes treatment, which was confirmed in NHEK cells and OSMs. Expression changes were not dependent on the $C$. acnes strain. However, we found that the extent of expression was dependent on $C$. acnes dose. Bacterial-induced changes in TNIP1 expression were regulated by signaling pathways involving NF- $\mathrm{B}$, p38, MAPKK and JNK. Experimental modification of TNIP1 levels affected constitutive and C. acnes-induced NF-kB promoter activities and subsequent inflammatory cytokine and chemokine mRNA and protein levels. These results suggest an important role for this negative regulator in the control of bacterially induced TLR signaling pathways in keratinocytes. We showed that all-trans retinoic acid (ATRA) induced elevated TNIP1 expression in HPV-KER cells and also in OSMs, where TNIP1 levels increased throughout the epidermis. ATRA also reduced constitutive and bacterium-induced levels of TNF $\alpha$, CCL5 and TLR2, while simultaneously increasing CXCL8 and TLR4 expression. Based on these findings, we propose that ATRA may exhibit dual effects in acne therapy by both affecting the expression of the negative regulator TNIP1 and attenuating TLR2-induced inflammation. Overall, TNIP1, as a possible regulator of $C$. acnes-induced innate immune and inflammatory events in keratinocytes, may play important roles in the maintenance of epidermal homeostasis. 


\section{INTRODUCTION}

The human skin harbors a specialized microbiome, which plays an important role in the regulation and maintenance of epidermal homeostasis. Various members of the microbiome are also important in the pathogenesis of several skin diseases, most of which lead to exaggerated innate immune and inflammatory events. Examples of pathogenesis involving bacteria present in the microbiome include Malassezia spp in seborrheic dermatitis, Staphylococcus aureus in atopic dermatitis and Cutibacterium acnes (C. acnes, formerly known as Propionibacterium acnes) in acne vulgaris (1-4).

C. acnes becomes a dominant species in the pilosebaceous units during puberty by colonizing the hair follicle region, often forming a biofilm $(5,6)$. Human epidermal keratinocytes sense the presence of this bacterium with pathogen recognition receptors. C. acnes recognition is mostly mediated by the activation of toll-like receptors (TLRs) 2 and 4, induction of the canonical signaling pathway and subsequent innate immune and inflammatory events $(7,8)$. An important mediator of this cascade is the NF- $\mathrm{B}$ transcription factor, which regulates the expression of key genes in the initialization and maintenance of downstream responses. These genes include different cytokines, such as tumor necrosis factor $\alpha(\mathrm{TNF} \alpha)$, interleukin (IL) $1 \alpha$, IL1 $\beta$, and IL6, chemokines, such as C-X-C motif chemokine ligand 8 (CXCL8) and C-C motif chemokine ligand 5 (CCL5), antibacterial peptides, such as human beta defensin 2 (hBD2), and other inflammatory mediators (9, 10). The innate immune activation of keratinocytes and the inflammatory milieu they generate in their environment favors the activation of other cell types, including sebocytes, dendritic cells and macrophages. Adaptive immune events are also induced, leading to the activation of the Th1/Th17 pathway $(11,12)$. Overall, these events contribute to the induction of the characteristic inflammatory symptoms during disease pathogenesis in adolescents. Inflammation and acne lesions are generally present transiently. In adolescents the inflamed follicles heal by themselves and the affected individuals often do not exhibit any residual signs. In the early twenties, even after resolution of the disease, C. acnes still dominates the microflora, especially in the sebum-rich skin regions. However, the bacterium does not usually provoke immune activation and inflammation in keratinocytes and/or other immune cells. This age-dependent response to $C$. acnes indicates different mechanisms controlling the downstream events induced by the bacterium.

Several negative regulators of the TLR and NF-кB signaling pathways have been identified in the past decade $(13,14)$. Through their regulatory functions, these negative regulators play important roles in the protection against extreme and prolonged activation of TLR and NF- $\kappa B$ downstream elements, both of which may lead to uncontrolled inflammation and tissue damage. TNIP1 (TNFAIP3 interacting protein 1), one such negative regulator, was identified as an interacting partner of TNFAIP3 (TNF alpha induced protein 3). TNIP1 has been shown to affect different signaling pathways by interacting directly with various proteins, including NEMO, TRAF1, p105, FADD, and
RIP1. As a consequence, the transcription factor NF- $\mathrm{KB}$ and the TLR-MYD88 signaling cascade is inhibited and apoptotic and autoimmune events are negatively affected $(15,16)$. TNIP1 is known to be expressed in keratinocytes, where this protein controls cell proliferation partly due to the regulation of ERK $1 / 2$ signaling (17). TNIP1 promoter activity may be regulated by NF- $\kappa B, P P A R$, retinoic acid receptors and also SP sites. These factors contribute to its basal and inducible activation possibly in a cell-type-specific manner (18-20). TNIP1's role in the regulation of TLR signaling in keratinocytes has been suggested in a recent study showing that its attenuation sensitizes $\mathrm{HaCaT}$ keratinocytes to synthetic TLR ligand treatment (21).

Currently, it is not clear whether negative regulators of the TLR and NF- $\mathrm{KB}$ signaling pathways play a role in the regulation of human microbiome-induced signaling events. Thus, we aimed to analyse the possible role of TNIP1 in C. acnes-induced innate immune and inflammatory events using in vitro cultured immortalized human keratinocytes and organotypic skin models (OSMs).

Our results show that, upon C. acnes treatment, TNIP1 expression is rapidly induced in a dose-dependent manner, and this induction is regulated by the NF- $\mathrm{KB}$ and JNK pathways and to some extent by p38- and MAPKK-dependent signaling pathways. In addition, modified TNIP1 levels affect the outcome of bacterium-induced molecular events, suggesting that this molecule acts through a negative regulatory feedback loop.

Retinoids, including all-trans retinoic acid (ATRA), are used as effective acne drugs and act by promoting cell proliferation, inhibiting keratinocyte terminal differentiation, decreasing sebum production and, as a result, indirectly reducing the amount of $C$. acnes (22). We aimed to investigate whether ATRA also affects the innate immune function of keratinocytes and found that ATRA treatment increases TNIP1 expression levels in our model systems. Our results reveal a possible mode of retinoid action that has not been explored previously.

\section{MATERIALS AND METHODS}

\section{Cell Cultures and Models}

The human immortalized keratinocyte cell line HPV-KER (23) and normal human epidermal keratinocytes (NHEK) were used for our experiments. Both cell types were cultured in keratinocyte serum-free medium (KSFM, Life Technologies, Carlsbad, United States) containing 1\% antibiotic/antimycotic (AB/AM, Sigma Aldrich, St. Louis, MO, United States) solution and supplemented with epidermal growth factor and brain pituitary extract under standard laboratory conditions $\left(37^{\circ} \mathrm{C}\right.$ in a humidified atmosphere containing $5 \% \mathrm{v} / \mathrm{v} \mathrm{CO}_{2}$ ).

NHEKs and ex vivo skin biopsies were obtained from skin specimens collected from the Plastic Surgery Unit of our department. Written informed consent was obtained from investigated individuals. The study was approved by the Human Investigation Review Board of the University of Szeged (PSOEDAFN-002, 23 February 2015, Szeged, Hungary) and complying with the ethical standards in accordance with the Helsinki Declaration. NHEK cells were isolated from the skin samples as described earlier (24). For ex vivo organotypic skin models 
(OSMs), $6 \mathrm{~mm}$ punch biopsies were washed first with normal saline solution (NSS) containing $2 \% \mathrm{AB} / \mathrm{AM}$, followed by a wash in $\mathrm{AB} / \mathrm{AM}$-free NSS. Subsequently, they were placed onto the upper chambers of Transwell ${ }^{\circledR}$ cell culture inserts (Corning, New York, United States) and kept at the air-liquid interphase. This way, the dermal part of the biopsies were in contact with DMEM F12 liquid culture medium (Lonza, Basel, Switzerland) supplemented with $10 \%$ fetal bovine serum (EuroClone, Milan, Italy) lacking $\mathrm{AB} / \mathrm{AM}$.

\section{Treatments}

For bacterial treatment, HPV-KER and NHEK cells were plated in $\mathrm{AB} / \mathrm{AM}$-free KSFM culture medium and co-cultured with live C. acnes strains belonging to different phylogenetic groups within the species (889, ATCC 11828, 6609) at various multiplicity of infection (MOI). Ex vivo skin models were treated with the $C$. acnes 889 strain at a density of $3 \times 10^{7} \mathrm{cfu} / \mathrm{cm}^{2}$ for 24 h. C. acnes strains were cultured and stored as previously described in detail (10).

To analyze the effect of the active form of retinoic acid, ATRA was dissolved in DMSO and a $10^{-6} \mathrm{M}$ concentration was applied to HPV-KER cells for $48 \mathrm{~h}$ before $C$. acnes challenge. ATRA was applied to OSMs at a $1.5 \times 10^{-6} \mathrm{M}$ concentration for $24 \mathrm{~h}$. As a control, cells were subjected to DMSO treatment without the active ingredient.

The selective inhibitors of JNK (sp 600125, $10 \mu \mathrm{M}$ ), NF$\kappa \mathrm{B}$ (Bay 11-7085, $10 \mu \mathrm{M}$ ), p38 (sb 203580, $10 \mu \mathrm{M}$ ), MAPKK (PD 098059, $20 \mu \mathrm{M}$ ), STAT1 (Fludarabine, $25 \mu \mathrm{M}$ ) and STAT3 (Stattic, $5 \mu \mathrm{M}$ ), or, as a control, DMSO were applied to the cells for $1 \mathrm{~h}$ (all reagents from Sigma Aldrich, St. Louis, MO, United States).

\section{Transfection, Plasmids and siRNA-Mediated Gene Silencing}

Transient transfection experiments were performed using the X-tremeGENE 9 DNA Transfection Reagent (Roche, Indiana, United States). For the overexpression studies, HPV-KER cells were plated in 12-well plates (100,000 cells/well), transfected for $24 \mathrm{~h}$ with $0.5 \mu \mathrm{g}$ empty pcDNA3.1 vector or pcDNA3.1TNIP1 vector into which TNIP1 cDNA sequences (OriGene Technologies, Inc., MD, United States) had been inserted. Transfection-grade plasmid was prepared using the E.Z.N.A Endo-free plasmid DNA Maxi Kit (Omega Bio-tek, Inc., GA, United States).

For siRNA-mediated gene silencing, siRNA was delivered by the Santa Cruz siRNA Transfection Reagent (Santa Cruz Biotechnology, Texas, United States) according to the manufacturer's instructions. ON-TARGETplus SMARTpool TNIP1 siRNA or ON-TARGETplus Non-targeting Pool (Dharmacon, Lafayette, United States) constructs were used at a concentration of $10 \mathrm{nM}$.

A NF- $\mathrm{B}$ luciferase reporter assay was performed using the PathDetect pNF-кB-Luc Cis-Reporter Plasmid (Stratagene, California, USA), pGL4.75[hRluc/CMV] Vector and the Firefly \& Renilla Dual Luciferase Assay Kit (Biotium Inc., California, United States), according to the manufacturer's instructions.

\section{ELISA}

C. acnes-treated and control HPV-KER cell culture supernatants were collected and the levels of secreted CXCL8 (R\&D Systems, Minneapolis, United States), IL-6 and CCL5 (PeproTech EC Ltd., London, United Kingdom) were measured according to the manufactures' instructions.

\section{RNA Isolation, cDNA Synthesis and Real-Time RT-PCR}

Total RNA was isolated using TRI-Reagent (Molecular Research Center; Cincinnati, United States). cDNA synthesis was performed using $1 \mu \mathrm{g}$ RNA with the iScript TM cDNA Synthesis kit (Bio-Rad, Hercules, United States). Changes in mRNA expression were detected by real-time RT-PCR using the Universal Probe Library (Roche, Indiana, United States) or the TaqMan Gene expression Assay (Thermo Scientific, Rockford, United States). Supplementary Table 1 lists the PCR protocols and primer sequences used. All data were normalized to the $18 \mathrm{~S}$ rRNA using the $\Delta \Delta \mathrm{C}_{\mathrm{t}}$ method and compared to the time-matched untreated control samples.

\section{Protein Isolation and Western Blot Analysis}

For the preparation of whole cell lysates, samples were collected and lysed in lysis buffer containing $20 \mathrm{mM}$ 4-(2-hydroxyethyl)1-piperazineethanesulfonic acid, $150 \mathrm{mM} \mathrm{KCl,} 1 \mathrm{mM} \mathrm{MgCl}_{2}$, $1 \mathrm{mM}$ DTT, 5\% Triton X-100, 10\% glycerol, 0.1\% NP-40, $1 \%$ Protease Inhibitor Cocktail, phenylmethylsulfonyl fluoride and $0.5 \%$ sodium dodecyl sulfate (SDS) (all from Sigma Aldrich, St. Louis, MO, United States). Protein concentrations were measured with the BCA Protein Assay Kit (Thermo Scientific, Rockford, United States). Samples (50 $\mu \mathrm{g}$ ) were separated on a $7.5 \%$ SDS polyacrylamide-gel electrophoresis and transferred to nitrocellulose membrane (Bio-Rad, Hercules, United States), blocked in Tris-buffered saline (TBS) containing 5\% Blotting-Grade Blocker (Bio-Rad, Hercules, United States). Membranes were incubated over-night at $4{ }^{\circ} \mathrm{C}$ with primary antiTNIP1 antibody diluted 1:500 and anti-actin (Sigma Aldrich, St. Louis, MO, United States) diluted 1:1,000. Subsequently, membranes were incubated for $2 \mathrm{~h}$ at room temperature with a horseradish peroxidase-conjugated anti-rabbit IgG (Santa Cruz Biotechnology, Texas, United States) secondary antibody diluted 1:2,000. Proteins were visualized with luminol (Bio-Rad, Hercules, United States) using a Omega Lum ${ }^{\mathrm{TM}}$ G Imaging System (Gel Company, CA, United States).

\section{Fluorescence Microscopic Analysis}

HPV-KER cells were grown on glass sides, fixed with $2 \%$ paraformaldehyde (PFA) for $5 \mathrm{~min}$, permeabilized with $0.1 \%$ Triton X, 2\% PFA-containing phosphate-buffered saline (PBS), and blocked for $2 \mathrm{~h}$ at room temperature with PBS containing $1 \%$ bovine serum albumin (BSA), $0.05 \%$ Triton X 100, and $10 \%$ goat serum. Cells were stained overnight at $4{ }^{\circ} \mathrm{C}$ with anti-human TNIP1 antibody (Sigma Aldrich, St. Louis, MO, United States) or rabbit IgG for isotype control. As a secondary antibody, Alexa Fluor 488 conjugated anti-rabbit IgG, was used for $2 \mathrm{~h}$. Filamentous actin was stained by Alexa Fluor $546^{\circledR}$ phalloidin (Life Technologies, Carlsbad, United States) diluted 1:100 in PBS 
containing 1\% BSA for $20 \mathrm{~min}$. Nuclei were stained for $10 \mathrm{~min}$ with $4^{\prime}, 6$-diamidino-2-phenylindole (DAPI) diluted 1:500.

Frozen sections of ex vivo skin models were pre-incubated with PBS for $5 \mathrm{~min}$ and fixed and permeabilized with Foxp3 staining buffer set (Thermo Scientific, Rockford, United States) and blocked for $1 \mathrm{~h}$ at room temperature with TBS containing $1 \%$ BSA and $1 \%$ normal goat serum. Cells were stained for $1 \mathrm{~h}$ with anti-human TNIP1 antibody or rabbit IgG for isotype control. As a secondary antibody, Alexa Fluor 546 conjugated anti-rabbit IgG (Thermo Scientific, Rockford, United States), was used for $1 \mathrm{~h}$ at room temperature. Nuclei were stained for $6 \mathrm{~min}$ with DAPI diluted 1:100.

\section{Statistical Analysis}

Unless otherwise noted, all data are presented as mean \pm standard error of the mean (SEM) for three independent experiments. For real-time RT-PCR analyzes and enzymelinked immunosorbent assay (ELISA), each treatment was performed at least in triplicate; for western blot and fluorescence microscopic analysis each treatment was performed once in every independent experiment. Data were compared using paired, twosample $t$-test with False Discovery Rate (FDR) correction. A probability value of less than 0.05 was considered significant.

\section{RESULTS}

\section{TNIP1 Is Expressed in Keratinocytes and its Expression Increases in Response to the Presence of $\boldsymbol{C}$. acnes}

Initially, we aimed to analyse changes in TNIP1 expression in in vitro cultured keratinocytes after treatment with $C$. acnes. For this purpose, NHEK or HPV-KER cells were treated with the bacterium. Keratinocyte to $C$. acnes ratios were determined in extensive preliminary studies. We chose conditions which induced relatively fast and reproducible cellular responses, but did not induce cell death in the time course of our studies (23).

We found that TNIP1 is expressed in HPV-KER cells and that mRNA expression rapidly and significantly increased after $C$. acnes 889 treatment $(\mathrm{MOI}=100)$, reaching the maximum at $12-$ $24 \mathrm{~h}$ (Figure 1A). These findings suggest that these cells recognize the presence of $C$. acnes bacterium.

It has been suggested that various $C$. acnes strains may have different effects on the cellular and molecular properties of human keratinocytes (10). We applied C. acnes strains (MOI $=100$ ) belonging to various phylogenetic groups within the species (C. acnes 889, 6609, ATCC 11828, Group IA, IB, and II.) and compared the effects. No strain-specific differences were observed: all of the $C$. acnes strains used induced similar changes in mRNA expression in HPV-KER cells (Figure 1B). Subsequently, only the C. acnes 889 strain was used in further experiments.

We also found, that bacterium-induced changes in TNIP1 expression were dose-dependent: the abundance of mRNA increased in parallel with increasing C. acnes 889 bacterial doses (Figure 1C).

Next, we analyzed TNIP1 protein levels in HPV-KER cells using western blot analysis and immunocytochemistry. Elevated
TNIP1 levels were detected in the $6 \mathrm{~h}$ samples and remained high during the time-course of the experiment (Figure 1D). Immunocytochemical staining of TNIP1 resulted in the presence of immunofluorescent dots, occurring mostly in the perinuclear region of HPV-KER cells. The number of labeled dots increased after $24 \mathrm{~h}$ of $C$. acnes treatment (MOI = 100) (Figure 1E). Similarly to the mRNA levels, the abundance of TNIP1 protein increased in parallel with increasing C. acnes 889 doses (Figure 1F).

To identify which signaling pathways are involved in the regulation of constitutive and $C$. acnes-induced TNIP1 expression levels in keratinocytes, we treated HPV-KER cells with specific inhibitors of well-known representatives (JNK, NF$\kappa \mathrm{B}, \mathrm{p} 38, \mathrm{MAPKK}, \mathrm{STAT} 1$, and STAT3) of different signaling pathways before the $C$. acnes treatment and analyzed subsequent changes in TNIP1 mRNA expression by real-time RT-PCR. We found that constitutive TNIP1 expression was significantly decreased when JNK and MAPKK were inhibited. Furthermore, C. acnes-induced changes in TNIP1 expression diminished in response to inhibition of JNK and MAPKK as well as of NF-кB and p38. In contrast, no effect was observed with STAT1 and STAT3 inhibition (Figure 1G).

To confirm that $C$. acnes-induced TNIP1 expression changes were not specific to the HPV-KER cell line, we repeated the experiments using NHEK cells and found similar results. TNIP1 mRNA levels increased in response to $C$. acnes treatment (Figure 1H), and increased protein levels were also noted in NHEK cell cultures $24 \mathrm{~h}$ after treatment (Figure 1I).

To confirm that the observed results were not specific to keratinocyte monolayer cultures, we also repeated the experiments using an ex vivo, OSMs. Punch biopsies of full thickness skin samples from healthy donors were cultured at the air-liquid interphase and C. acnes 889 was applied to the top (epidermal side) of the samples. We found that TNIP1 protein was expressed throughout the epidermis, and that slightly higher levels were detected in the less differentiated, basal layers. Within the keratinocytes, localization was primarily cytoplasmic and perinuclear. Elevated TNIP1-staining levels were found $24 \mathrm{~h}$ after C. acnes treatment (Figure 1J).

\section{TNIP1 Down-Regulates Both Constitutive and $C$. acnes-Induced Inflammatory Cytokines and Chemokines Expression}

To analyze the role of TNIP1 in the regulation of C. acnesinduced inflammatory events, we experimentally modified endogenous TNIP1 levels with cDNA-based transient overexpression or siRNA-mediated silencing. We monitored the expression of selected pro-inflammatory cytokines and chemokines that are known downstream targets of the TLR signaling pathway, as well as the promoter activity of the NF- $\kappa$ B transcription factor, using real-time RT-PCR, ELISA analysis and a luciferase- reporter assay.

cDNA-based transient overexpression resulted in markedly increased TNIP1 protein levels in HPV-KER cells (Figure 2A). As a consequence, significantly decreased constitutive and $C$. acnes-induced NF- $\kappa \mathrm{B}$ promoter activities were measured in the subsequent luciferase reporter assay (Figure 2B). mRNA 
A

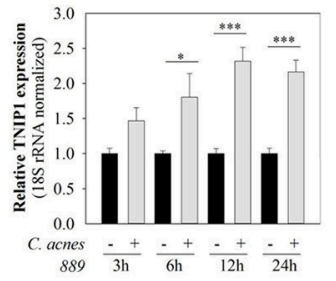

D
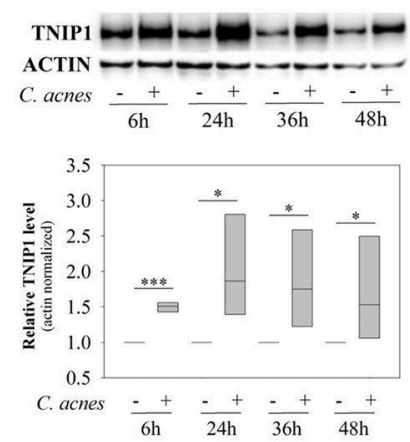

G

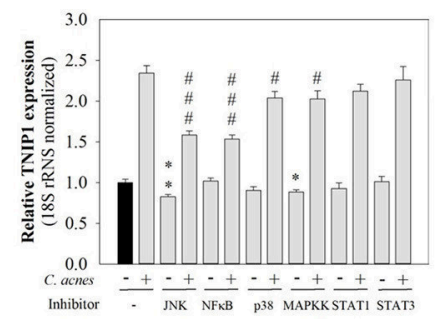

B

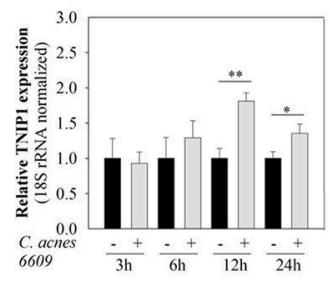

E
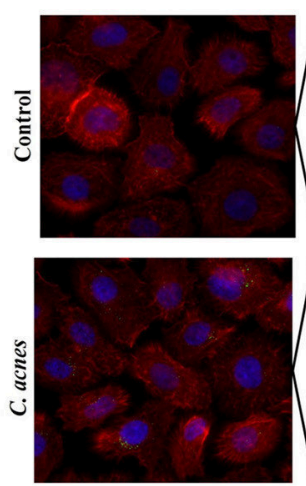

H

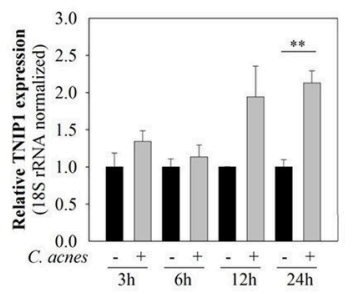

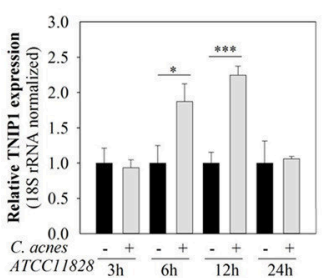

C

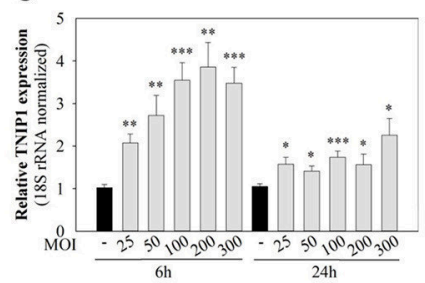

F
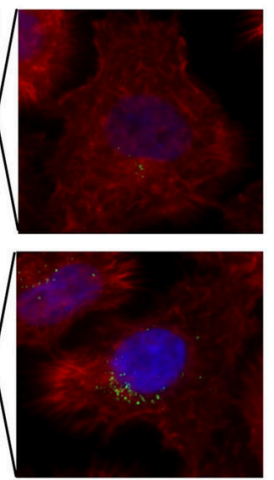

I

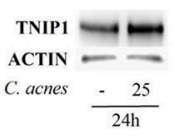

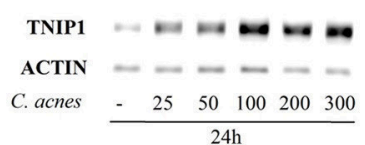

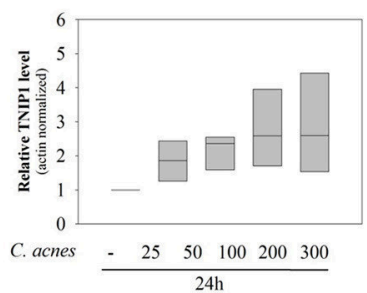

J
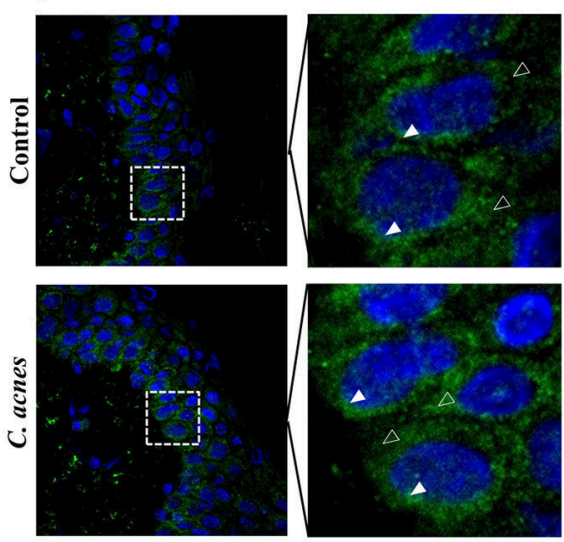

FIGURE 1 | mRNA and protein expression of TNIP1 increases in response to C. acnes treatment. HPV-KER cells (A-G), NHEK cells (H,I) and an ex vivo skin model (J) were treated with C. acnes 889 at a MOI of 100, unless otherwise indicated, and changes in mRNA and protein levels were analyzed. (A,B,C,H) Changes in TNIP1 mRNA expression were analyzed by real-time RT-PCR; data were normalized to $18 \mathrm{~S}$ rRNA using the $\Delta \Delta \mathrm{C}_{t}$ method and compared to the time-match untreated control values. Error bars are SEM. Statistical analyzes: paired, two-sample $t$-test with FDR correction: ${ }^{*} p<0.05,{ }^{* *} p<0.01,{ }^{* \star *} p<0.001$. (G) HPV-KER cells were plated and pretreated with selective inhibitors for JNK, NF-kB, p38, MAPKK, STAT1 and STAT3 or, as a control, DMSO for $1 \mathrm{~h}$. C. acnes challenge was performed for $12 \mathrm{~h}$ and samples were collected for subsequent mRNA analyzes by real-time RT-PCR. All data were normalized to the $18 \mathrm{~S}$ rRNA using the $\Delta \Delta \mathrm{C}_{t}$ method and compared to DMSO-treated control samples. Statistical analyzes: paired, two-sample $t$-test with FDR correction, basal TNIP1 expression ${ }^{\star} p<0.05,{ }^{\star \star} p<0.01$ or $C$. acnes-induced TNIP1 expression, \#p <0,05, \#\#\#p<0.001. were compared. (D,F) TNIP1 protein was detected with western blot analysis and quantitated using an Image Pro Plus, where all data were normalized to actin; a representative blot is shown. Statistical analyzes: paired, two-sample $t$-test: ${ }^{*} p<0.05$. (E) After $24 \mathrm{~h} C$. acnes treatment, HPV-KER cells were visualized with immunofluorescence staining for TNIP1 (green), Phalloidin (red) and DAPI (blue). (I) NHEK cells were treated with C. acnes and TNIP1 protein was detected with western blot analyzes (a representative experiment). (J) OSMs were treated with $3 \times 10^{7}$ bacterium for $24 \mathrm{~h}$, and cells were visualized with immunofluorescence staining for TNIP1 (green) and DAPI (blue), where empty arrows indicate cytoplasmic location and filled arrows indicated perinuclear localization.

expression of constitutive and C. acnes-induced TNF $\alpha$, CXCL8 and CCL5 also decreased compared to the empty-vector transfected samples. In addition, bacterium-induced mRNA levels of IL6 decreased, whereas IL1 $\alpha$ levels were not affected
(Figure 2C). Overexpression of TNIP1 also decreased IL-6, CXCL8, and CCL5 protein secretion (Figure 3A).

In contrast, siRNA-mediated silencing of TNIP1 led to markedly decreased TNIP1 protein levels (Figure 2D). As a 
TNIP1 overexpression

A

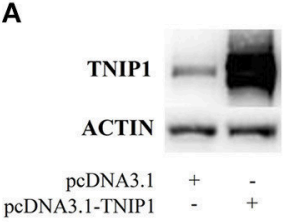

B pcDNA3.1-TNIP
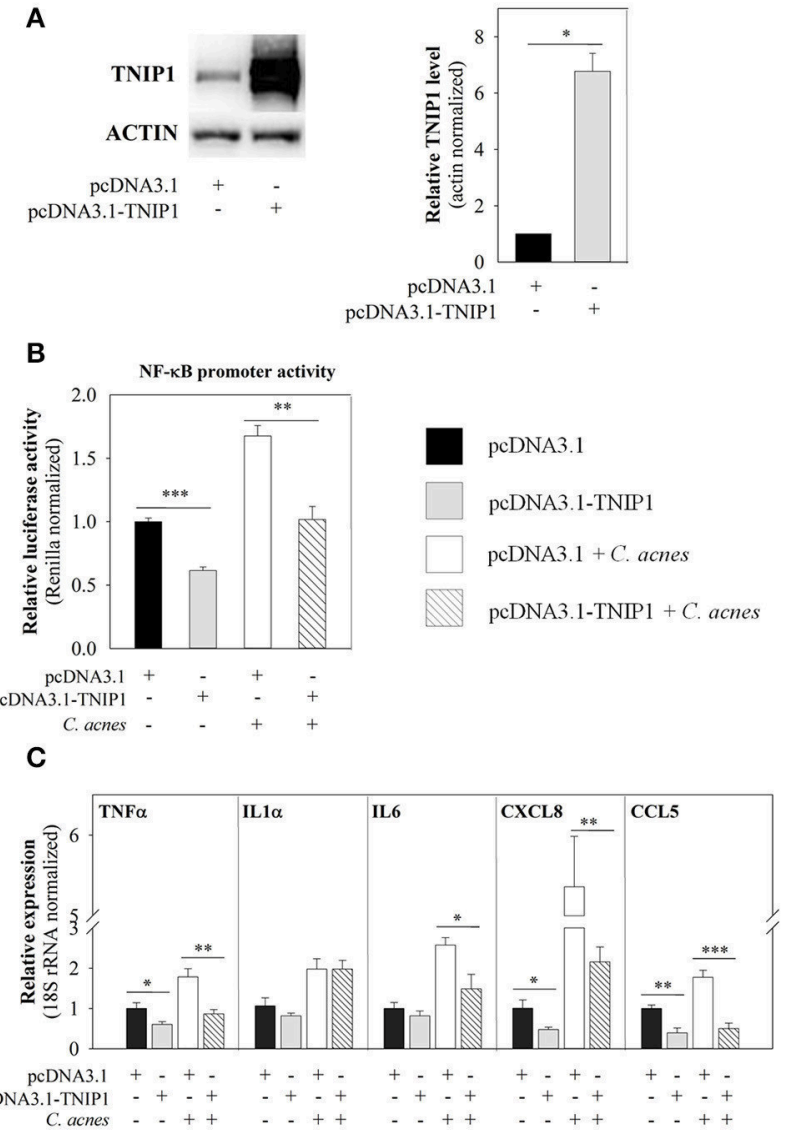

TNIP1 silencing

D

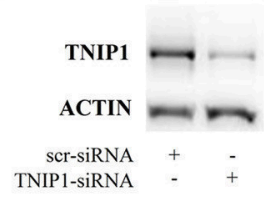

E
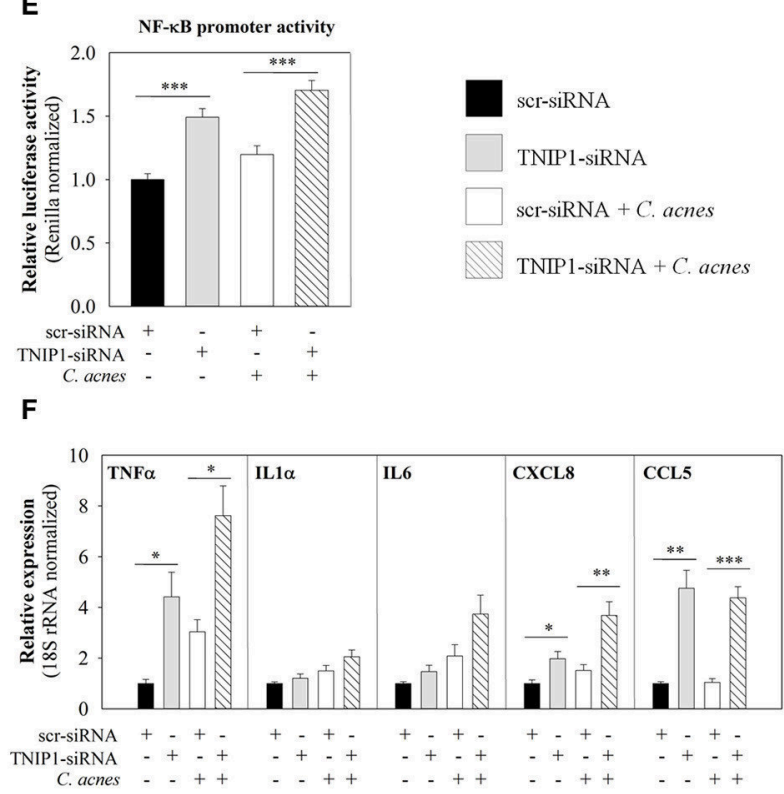

FIGURE 2 | Effects of TNIP1 levels on the downstream targets of the TLR signaling pathway. After plating HPV-KER cells, TNIP1 overexpression (24 h) (left panel) or siRNA-mediated silencing $(48 \mathrm{~h}$ ) (right panel) was performed. Subsequently, cells were treated with $\mathrm{C}$. acnes $(\mathrm{MOI}=100)$ for $6 \mathrm{~h}$. (A,D) TNIP1 protein levels were analyzed by western blot after overexpression or silencing and quantitated using Image Pro Plus, where all data were normalized to actin; a representative blot is shown. Error bars are SD. (B,E) NF-кB promoter activities were measured with a luciferase reporter assay; data were normalized to signal from a vector constitutively expressing Renilla luciferase and compared to time-matched samples transfected with vectors that were empty (pcDNA3.1) or contained scr-siRNA. (C,F) mRNA levels of TNF $\alpha$, IL-6, CXCL8 and CCL5 were analyzed by real-time RT-PCR; data were normalized to 18S rRNA using the $\Delta \Delta C_{t}$ method and compared to time-matched samples transfected with vectors that were empty (pcDNA3.1) or contained scr-siRNA. Error bars are SEM. Statistical analyzes: paired, two-sample $t$-test: ${ }^{\star} p<0.05,{ }^{\star \star} p<0.01,{ }^{\star \star \star} p<0.001$.

consequence, significantly increased constitutive and bacterial induced NF- $\mathrm{B}$ promoter activities were found in a luciferase reporter assay (Figure $2 \mathrm{E}$ ). Constitutive and $C$. acnes-induced expression of TNF $\alpha$, CXCL8, and CCL5 mRNA markedly increased, whereas IL1 $\alpha$ and IL6 expression increased moderately in response to TNIP1 silencing (Figure 2F). Constitutive and bacterium-induced secretion of CXCL8, IL6, and CCL5 were also elevated in TNIP1 silenced HPV-KER cells (Figure 3B).

\section{ATRA Induces TNIP1 Expression and Affects the Levels of Downstream Targets of the TLR Signaling Pathway}

The TNIP1 promoter contains retinoic acid response elements (RARE) and retinoid-acid-receptor binding sites. These elements are involved in the induction of TNIP1 expression by retinoic acid under permissive epigenetic conditions in different cell lines
(19). Since ATRA is an effective drug used for acne treatment, we examined whether this compound is capable of regulating TNIP1 and, thus, the expression of downstream targets of the TLR signaling pathway in keratinocytes.

We observed that ATRA treatment led to slightly elevated TNIP1 mRNA levels, although this effect was not statistically significant (Figure 4A), and to significantly increased TNIP1 protein expression (Figure 4B). In addition, constitutive and C. acnes-induced mRNA expression of TLR-2 and the proinflammatory TNF $\alpha$ and CCL5 decreased upon ATRA treatment. In contrast, both constitutive and C. acnes-induced TLR-4 and CXCL8 mRNA expression levels increased, whereas TLR3 and IL-6 mRNA levels were not affected by the drug (Figures 4D,E).

To confirm that the effects of ATRA on TNIP1 expression were not specific to the HPV-KER cell line, we also applied the drug to the upper, epidermal part of OSMs. We found that TNIP1 


\section{A}

\section{TNIP1 overexpression}
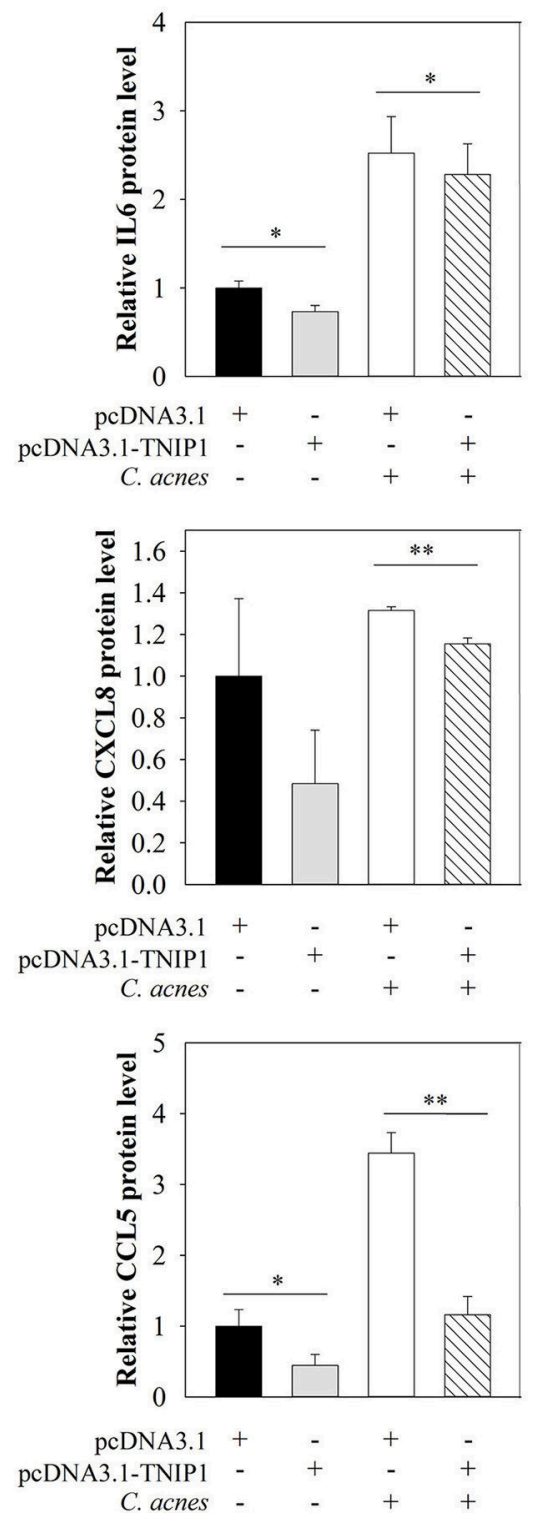

B TNIP1 silencing

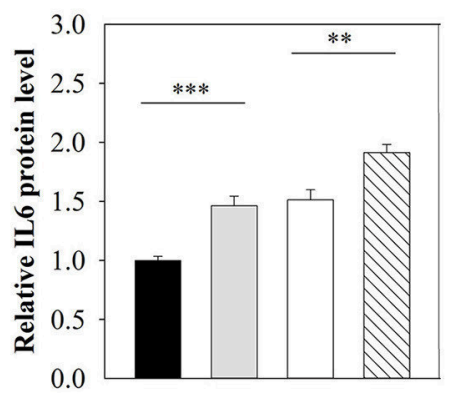

$\begin{array}{rllll}\text { Scr-siRNA } & + & - & + & - \\ \text { IPl-siRNA } & - & + & - & +\end{array}$

C. acnes - $\quad$ - $\quad+$
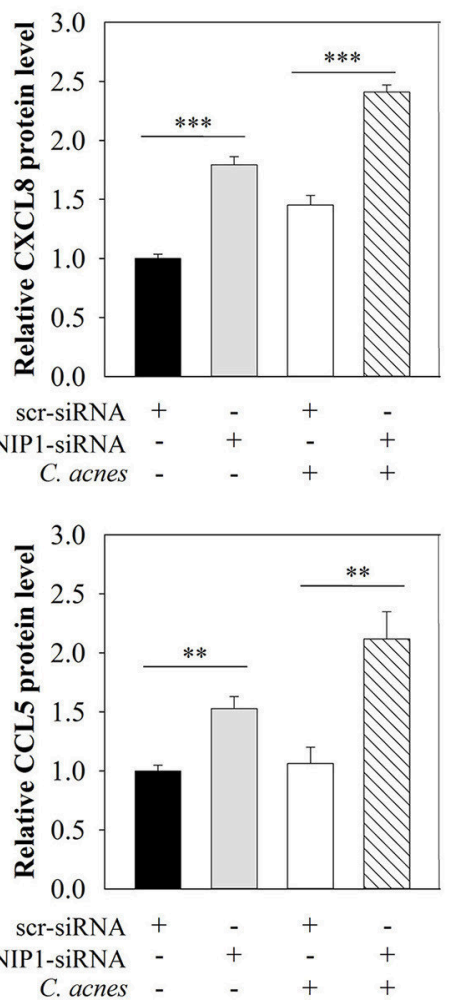

FIGURE 3 | Effects of TNIP1 levels on secretion of pro-inflammatory cytokine and chemokine. After plating HPV-KER cells, (A) TNIP1 overexpression (24 h) or (B) siRNA-mediated silencing ( $48 \mathrm{~h}$ ) was performed. Subsequently, cells were treated with $\mathrm{C}$. acnes $(\mathrm{MOI}=100)$ for $24 \mathrm{~h}$. Secreted cytokine and chemokine levels were measured by ELISA and relative protein levels are presented. Data were normalized to time-matched samples transfected with a vector that was empty (pcDNA3.1) or contained scr-siRNA. Values of the measured protein levels were the following: IL-6: 200-1,300 pg/ml, CXCL8: 200-2,800 pg/ml, CCL5: 50-400 pg/ml. Error bars are SEM. Statistical analyzes: paired, two-sample $t$-test: ${ }^{\star} p<0.05,{ }^{\star \star} p<0.01,{ }^{\star \star \star} p<0.001$.

protein expression levels had increased in all epidermal layers $24 \mathrm{~h}$ after ATRA treatment (Figure 4C), in a manner similar to observed for immortalized keratinocytes.

\section{DISCUSSION}

Human skin harbors a special microbiota which protects against pathogens, helps to maintain the proper functions of the skin and also serves as an immunological barrier, functions that have also been proposed for the gut microbiota (25). These skin functions are partly achieved by keeping the immune system at a basal activated state. Low levels of TLR activation may contribute to the maintenance of basal expression from genes with important roles for the immunological barrier (e.g., inducible antimicrobial peptides, such as hBD2) (26).

Under healthy conditions, these events do not lead to inflammatory processes. However, under certain conditions, the commensal bacteria are involved in inflammatory skin diseases, 


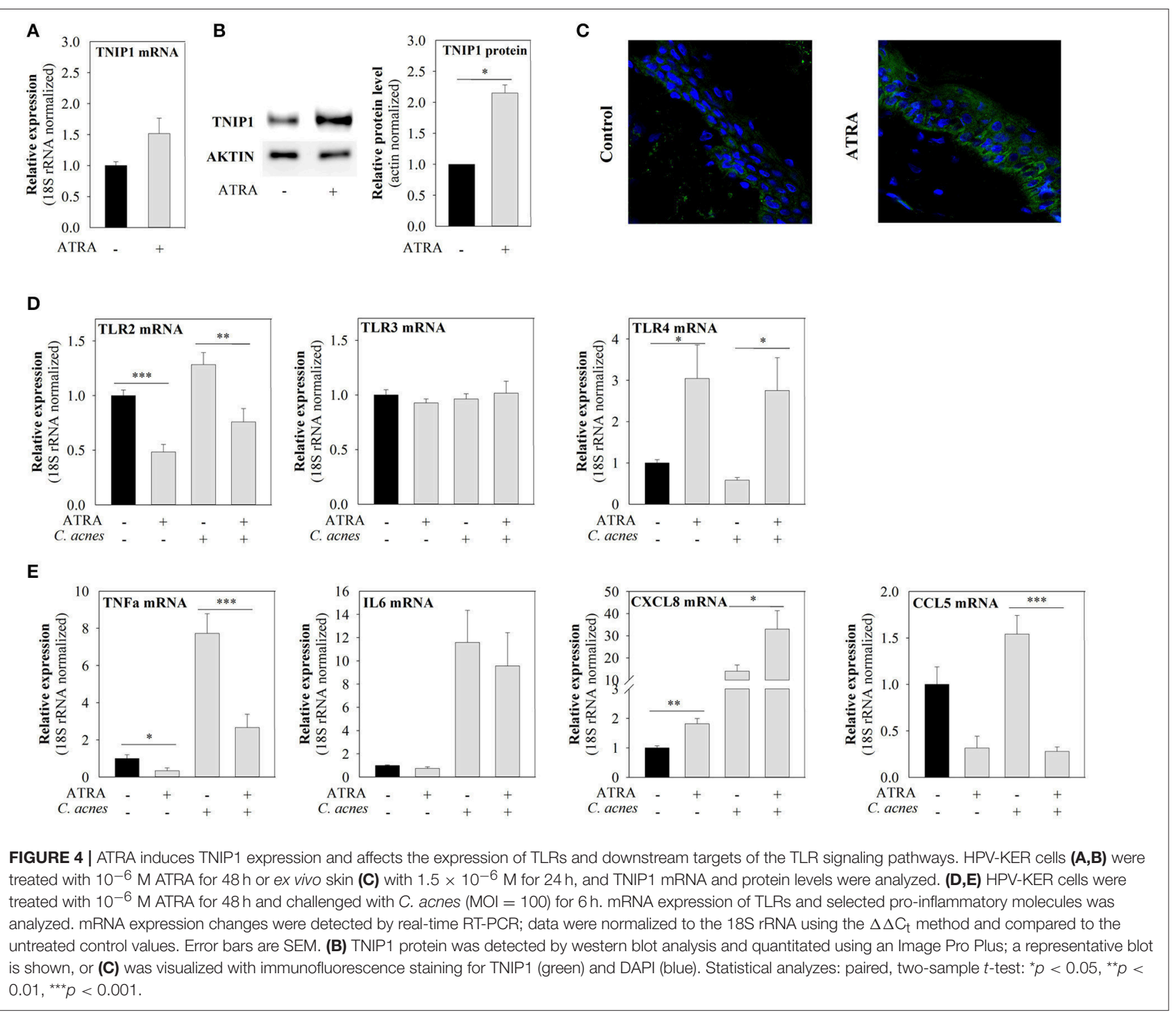

such as acne $(27,28)$. The mechanisms underlying this duality the protective and pathogenic aspects of the continual presence of the skin microbiota - are still unclear $(1,25)$ C. acnes, a dominant member of the human skin microbiome, is recognized by TLR2 and TLR4 in human epidermal keratinocytes. This bacterium can induce innate immune and inflammatory events, such as production of inflammatory cytokines, chemokines, antimicrobial peptides and other inflammatory mediators, as well as autophagy in keratinocytes, and, through this induction, plays a role in the pathogenesis of acne vulgaris $(7,8,27,29)$. However, at the beginning of early adolescence this bacterium is overwhelming dominant in the skin microbiota under healthy conditions, yet does not induce macroscopically detectable inflammation $(3,5)$. These observations suggest that one or more mechanisms regulate $C$. acnes-induced immune activation.

In the past decades, several negative regulators of TLR and the NF-kB signaling pathway have been identified. The regulatory functions of these molecules play important roles in the protection against exaggerated immune activation. Since their discovery, an increasing number of publications has reported correlation between genetic variations of these proteins and altered expression and/or dysfunctional proteins as well as with diseases associated with inflammation, such as psoriasis, systemic lupus erythematosus and cancer $(13,16,30)$. Taken together, these results suggest that proper regulation and function of these factors are indispensable for the maintenance of health. The role of these molecules in the assuagement of microbiome-induced immune activation has not yet been investigated.

In our study, we analyzed TNIP1, a regulator of NF- $\mathrm{B}$ signaling pathways, and found that it is a possible negative regulator of $C$. acnes-induced inflammatory events in HPVKER cells. TNIP1 is expressed in cultured keratinocytes and mRNA and protein expression increased in response to C. acnes treatment; the extent of activation was dependent on the bacterial 
dose. We confirmed that these changes were not specific for the HPV-KER cell line, as similar results were obtained using NHEK cells. In ex vivo OSMs we showed that TNIP1 is expressed in all epidermal layers, which was consistent with previous studies (17). We detected mostly cytoplasmic and perinuclear staining within the keratinocytes, in contrast to previous studies, where cytoplasmic and also intensive nuclear staining of TNIP1 was observed in $\mathrm{HaCaT}$ cells $(17,31)$. In response to $C$. acnes treatment, elevated TNIP1 levels were found in the epidermis. These results are in agreement with our findings using NHEK and HPV-KER cells: TNIP1 expression changes in keratinocytes in response to the presence of the bacterium. These findings show that, in addition to pro-inflammatory mediators, C. acnes also induces the expression of anti-inflammatory factors, possibly to avoid excessive inflammation and immune activation. Our results strongly suggest that TNIP1 may be one of the negative regulators of C. acnes-induced molecular events.

Earlier studies revealed differences in C. acnes straincomposition from healthy individuals and acne patients (32). It has been suggested that strains belonging to various phylogenetic groups within the species differentially affect the cellular and molecular properties of keratinocytes (10). In the present study, we did not observe strain-specific differences in TNIP1 expression in response to $C$. acnes, suggesting that strain-specific activation of keratinocytes are not related to the ability of the bacterium to induce the studied negative regulators.

In silico analyzes predicted the presence of putative biding sites for NF- $\kappa \mathrm{B}, \mathrm{AP}-1, \mathrm{SP}$ and $\mathrm{C} / \operatorname{EBP} \beta(33,34)$ in the TNIP1 promoter region. Gene expression and ChIP assays have confirmed that NF- $\kappa \mathrm{B}$ and SP1 binding sites are active in HeLa cells $(18,20)$. Our finding that specific JNK, NF- $\kappa$, p38 and MAPKK inhibitors decreased the bacterium-induced upregulation of TNIP1 suggest that these signaling pathways may be involved in the regulation of C. acnes-induced TNIP1 expression in keratinocytes. Moreover, the intricate pattern of TNIP1 regulation suggests this molecule may have important roles in different processes.

To confirm the negative regulatory role of TNIP1 in C. acnesinduced molecular events, we modified its endogenous levels by cDNA-based overexpression and siRNA-mediated silencing and examined the expression of downstream elements of TLR signaling pathways. NF- $\kappa \mathrm{B}$, one of the main mediator of signaling cascades activated in our experiments, is induced upon exposure to bacteria $(7,10)$. Our results, that TNIP1 overexpression decreased basal and C. acnes-induced NF-кB promoter activities and mRNA levels of TNF $\alpha$, CXCL8 and CCL5 as well as secretion of CXCL8, IL-6 and CCL5 correlate well with findings on other cell types. In HeLa cells, TNIP1 overexpression inhibited constitutive TNF $\alpha$, IL1 $\alpha$ expression and lipopolisaccharide (LPS)-induced activation of NF- $\kappa \mathrm{B}(15,35)$.

In TNIP1-silenced cells, we observed increased constitutive NF- $\kappa \mathrm{B}$ promoter activities and elevated pro-inflammatory cytokine and chemokine mRNA and protein levels. TNIP1 silencing also increased C. acnes-induced NF- $\mathrm{B}$ promoter activity, mRNA levels and secretion of the mediators mentioned above. These results further support the conclusion that TNIP1 plays a role in the regulation of $C$. acnes-induced events in HPV-KER cells and possibly in the maintenance of homeostatic conditions. In a recent report, the authors have also demonstrated that TNIP1-silenced HaCaT cells were hypersensitive to synthetic TLR3 and TLR2/6 ligands and subsequently increased JNK and p38 phosphorylation and nuclear translocation of NF- $\kappa$ B. They also observed increased levels of secreted IL6 and CXCL8 compared to control cells (21). Overall, these data indicate a negative regulatory role of TNIP1 in TLR signaling events in keratinocytes.

Other reports have also shown the importance of TNIP1 as a gatekeeper in NF- $\mathrm{B}$, JNK and p38 mediated processes, including the prevention of fetal liver apoptosis in a murine model and $\mathrm{TNF} \alpha$-induced apoptosis in different cell lines (36, 37). In contrast, although TNIP1-deficient mice develop a progressive, lupus-like inflammatory disease, isolated TNIP1deficient macrophages and dendritic cells showing no differences in pro-inflammatory signaling pathways with respect to IкB $\alpha$ degradation and resynthesis and phosphorylation of different MAPKs (p38, ERK1/2, and JNK1/2) compared to wild type mice upon CpG-DNA or TLR4 (LPS) stimulation $(38,39)$. These findings suggest that the role of TNIP1 during TLR activation might be cell-type specific and depends on the nature of the stimuli.

Earlier studies found functional RARE elements in the TNIP1 promoter region, and that TNIP1 was induced by ATRA in HeLa cells under permissive epigenetic conditions, in the presence of Trichostatin A co-treatment (19). Trichostatin A inhibits histone deacetylase I and II and alters gene expression by opening chromatin and allowing transcription factors to bind, thus, promoting transcription of different genes. We applied ATRA, an active form of retinoic acid, to HPV-KER cells and found that TNIP1 mRNA and protein expression levels increased in response to the treatment without the addition of chromatin-modification agents. Furthermore, treatment of OSMs led to similar results throughout the entire epidermis, suggesting that these effects were not specific to HPV-KER cells. It is not currently clear why HeLa cells behaved differently; however, differences in the duration of ATRA exposure or in the responsiveness of the different cells used might be responsible.

Despite the fact that retinoids, including ATRA, are widely used as an effective drug for acne therapy, the exact mechanism of action is not completely understood. Retinoids have been shown to promote cell proliferation, inhibit keratinocyte terminal differentiation, decrease the size of sebaceous glands and, indirectly, reduce the amount of $C$. acnes (40). Studies of the effect of retinoids on innate immunity are limited, and the results are often dependant on the cells used (19, 41-44). In most cases, ATRA application to monocytes and macrophages decreased TLR2 abundance as well as the expression of selected pro-inflammatory mediators; however, the mechanism of these effects remains unclear $(43,45,46)$.

In our experiments, ATRA treatment decreased the level of constitutive and $C$. acnes-induced pro-inflammatory mediators $\mathrm{TNF} \alpha$ and CCL5, but increased CXCL8 levels. These results are consistent with the findings of others; similar CXCL8 expression changes were observed in NHEK and also in other cell types in response to ATRA $(41,42,47)$. In NHEK cells, NF- $\kappa B$ and p38 signaling might contribute to these events (41). 
Based on all these data, we conclude that ATRA may regulate TNIP1 expression and, as a consequence, negatively affect C. acnes-induced inflammatory events in keratinocytes. Our proposed model may offer a possible, novel mode of retinoid action in acne treatment.

TLR2 expression is increased in the epidermis and monocytes isolated from acne patients compared to healthy controls, and this increase might be a result of $C$. acnes-induced inflammatory events. ATRA treatment decreased TLR2 levels in monocytes isolated from both healthy donors and acne patients following isotretinoin therapy, although TLR4 expression was not affected (7, 45, 46, 48, 49). We found similar changes in expression for TLR2 (decreased) and TLR4 (increased) in HPV-KER cells, suggesting that ATRA has opposite effects on these two receptors in keratinocytes.

We propose that the increased bacterial load in acneprone follicles is a factor leading to the formation of inflammatory symptoms. Retinoids may attenuate C. acnesinduced inflammation by decreasing the sizes of sebaceous glands and sebum secretion and, subsequently, control the $C$. acnes load. In addition, TLR2 levels are also attenuated, preventing the sensing of this Gram-positive bacterium, which leads to deleterious inflammation. By decreasing the expression of TLR2, an important gatekeeper of the skin, the risk of opportunistic infections is increased. To modulate this effect, expression of another gatekeeper, TLR4 is elevated. Thus, multiple levels of sensing and signal transduction may be available in the defense against potentially harmful microbial invaders. The fact that opportunistic bacterial and fungal infections are rare in the lesional skin of acne patients is consistent with our hypothesis.

TNIP1 may regulate $C$. acnes-induced signaling events through the establishment of a negative-regulatory feedback loop controlling NF- $\kappa \mathrm{B}$ activity in keratinocytes. Other signaling pathways, such as nuclear-receptor signaling cascades that are activated by retinoids, may also affect TNIP1 levels, which in turn can modify the outcome of the induced processes. Based

\section{REFERENCES}

1. Gallo RL, Nakatsuji T. Microbial symbiosis with the innate immune defense system of the skin. J Invest Dermatol. (2011) 131:1974-80. doi: 10.1038/jid.2011.182

2. Littman DR, Pamer EG. Role of the commensal microbiota in normal and pathogenic host immune responses. Cell Host Microbe (2011) 10:311-23. doi: 10.1016/j.chom.2011.10.004

3. Szabó K, Erdei L, Bolla BS, Tax G, Bíró T, Kemény L. Factors shaping the composition of the cutaneous microbiota. Br J Dermatol. (2017) 176:344-51. doi: 10.1111/bjd.14967

4. Scholz CFP, Kilian M. The natural history of cutaneous propionibacteria, and reclassification of selected species within the genus propionibacterium to the proposed novel genera acidipropionibacterium gen. Nov., cutibacterium gen. nov. and pseudopropionibacterium gen. nov. Int J Syst Evol Microbiol. (2016) 66:4422-32. doi: 10.1099/ijsem.0.001367

5. Oh J, Conlan S, Polley E, Segre JA, Kong HH. Shifts in human skin and nares microbiota of healthy children and adults. Genome Med. (2012) 4:77. doi: $10.1186 /$ gm 378

6. Grice EA, Segre JA. The skin microbiome. Nat Rev Microbiol. (2011) 9:244-53. doi: $10.1038 /$ nrmicro2537 on our results, TNIP1 may function as a negative regulator in keratinocytes that controls bacterium-induced inflammatory events, playing an important role in maintaining the homeostasis between skin cells and the skin microbiome. Development of novel, well-tolerated, TNIP1-specific acne therapeutic modalities could potentially reduce inflammation without the harmful side effects of currently available treatment options (antibiotic, retinoid or hormonal formulation usage).

\section{AUTHOR CONTRIBUTIONS}

$\mathrm{LE}$ and $\mathrm{KS}$ designed the experiments. LE, BB, RB, and GT performed the experiments. EU cultured and provided C. acnes strains, which were used in our experiments. LE, LK, and KS performed data analysis and wrote the manuscript. LK and KS were involved in the coordination of the study.

\section{FUNDING}

This work was supported by the GINOP-2.3.2-15-2016-00015 and OTKA NK105369 research grants. KS is a recipient of the János Bolyai Research Scholarship from the Hungarian Academy of Sciences. The funders had no role in study design, data collection and analysis, decision to publish, or preparation of the manuscript.

\section{ACKNOWLEDGMENTS}

We wish to thank Tanácsné Bajkán Andrea for her excellent technical assistance.

\section{SUPPLEMENTARY MATERIAL}

The Supplementary Material for this article can be found online at: https://www.frontiersin.org/articles/10.3389/fimmu. 2018.02155/full\#supplementary-material
7. Kim J, Ochoa M-T, Krutzik SR, Takeuchi O, Uematsu S, Legaspi AJ, et al. Activation of toll-like receptor 2 in acne triggers inflammatory cytokine responses. J Immunol. (2002) 169:1535-41. doi: 10.4049/jimmunol.169. 3.1535

8. Pivarcsi A, Bodai L, Réthi B, Kenderessy-Szabó A, Koreck A, Széll M, et al. Expression and function of toll-like receptors 2 and 4 in human keratinocytes. Int Immunol. (2003) 15:721-30. doi: 10.1093/intimm/dxg068

9. Koreck A, Pivarcsi A, Dobozy A, Kemény L. The role of innate immunity in the pathogenesis of acne. Dermatology (2003) 206:96-105. doi: $10.1159 / 000068476$

10. Nagy I, Pivarcsi A, Koreck A, Széll M, Urbán E, Kemény L. Distinct strains of propionibacterium acnes induce selective human $\beta$-defensin2 and interleukin-8 expression in human keratinocytes through toll-like receptors. J Invest Dermatol. (2005) 124:931-8. doi: 10.1111/j.0022-202X.2005. 23705.x

11. Mattii M, Lovászi M, Garzorz N, Atenhan A, Quaranta M, Lauffer F, et al. Sebocytes contribute to skin inflammation by promoting the differentiation of T helper 17 cells. Br J Dermatol. (2018) 178:722-30. doi: 10.1111/bjd.15879

12. Lovászi M, Mattii M, Eyerich K, Gácsi A, Csányi E, Kovács D, et al. Sebum lipids influence macrophage polarization and activation. Br J Dermatol. (2017) 177:1671-82. doi: 10.1111/bjd.15754 
13. Liew FY, Xu D, Brint EK, O'Neill LAJ. Negative regulation of toll-like receptor-mediated immune responses. Nat Rev Immunol. (2005) 5:446-58. doi: $10.1038 /$ nri1630

14. Miggin SM, O'Neill LAJ. New insights into the regulation of TLR signaling. $J$ Leukoc Biol. (2006) 80:220-6. doi: 10.1189/jlb.1105672

15. Mauro C, Pacifico F, Lavorgna A, Mellone S, Iannetti A, Acquaviva R, et al. ABIN-1 binds to NEMO/IKK $\gamma$ and co-operates with A20 in inhibiting NF- $\kappa$ B. J Biol Chem. (2006) 281:18482-88. doi: 10.1074/jbc.M601502200

16. G'Sell RT, Gaffney PM, Powell DW. A20-binding inhibitor of NF-??B activation 1 is a physiologic inhibitor of NF-??B: a molecular switch for inflammation and autoimmunity. Arthritis Rheumatol. (2015) 67:2292-302. doi: 10.1002/art.39245

17. Chen Y, Yan H, Song Z, Chen F, Wang H, Niu J, et al. Downregulation of TNIP1 expression leads to increased proliferation of human keratinocytes and severer psoriasis-like conditions in an imiquimod-induced mouse model of dermatitis. PLoS ONE (2015) 10:1-18. doi: 10.1371/journal.pone.0127957

18. Gurevich I, Zhang C, Encarnacao PC, Struzynski CP, Livings SE, Aneskievich BJ. PPAR $\gamma$ and NF- $\kappa \mathrm{B}$ regulate the gene promoter activity of their shared repressor, TNIP1. Biochim Biophys Acta (2012) 1819:1-15. doi: 10.1016/j.bbagrm.2011.09.006

19. Gurevich I, Zhang C, Francis N, Struzynsky CP, Livings SE, Aneskievich BJ. Human $\mathrm{TNF} \alpha$-induced protein 3-interacting protein 1 (TNIP1) promoter activation is regulated by retinoic acid receptors. Gene (2013) 515:42-8. doi: 10.1016/j.gene.2012.11.041

20. Encarnacao PC, Ramirez VP, Zhang C, Aneskievich BJ. Sp sites contribute to basal and inducible expression of the human TNIP1 (TNF $\alpha$-inducible protein 3-interacting protein 1) promoter. Biochem J. (2013) 452:519-29. doi: 10.1042/BJ20121666

21. Rudraiah S, Shamilov R, Aneskievich BJ. TNIP1 reduction sensitizes keratinocytes to post-receptor signalling following exposure to TLR agonists. Cell Signal. (2018) 45:81-92. doi: 10.1016/j.cellsig.2018.02.004

22. Layton A. The use of isotretinoin in acne. Dermatoendocrinol (2009) 1:162-9. doi: 10.4161/derm.1.3.9364

23. Tax G, Urbán E, Palotás Z, Puskás R, Kónya Z, Bíró $T$, et al. Propionic acid produced by propionibacterium acnes strains contri butes to their pathogenicity. Acta Derm Venereol. (2016) 96:43-49. doi: 10.2340/00015555-2154

24. Danis J, Göblös A, Bata-Csörgó Z, Kemény L, Széll M. Prins non-coding rna regulates nucleic acid-induced innate immune responses of human Keratinocytes. Front Immunol. (2017) 8:1053. doi: 10.3389/fimmu.2017.01053

25. Chu H, Mazmanian SK. Innate immune recognition of the microbiota promotes host-microbial symbiosis. Nat Immunol. (2013) 14:668-75. doi: 10.1038/ni.2635

26. Kinnebrew MA, Pamer EG. Innate immune signaling in defense against intestinal microbes. Immunol Rev. (2012) 245:113-31. doi: 10.1111/j.1600-065X.2011.01081.X

27. Beylot C, Auffret N, Poli F, Claudel J-P, Leccia M-T, Del Giudice P, et al. Propionibacterium acnes: an update on its role in the pathogenesis of acne. J Eur Acad Dermatol Venereol. (2014) 28:271-8. doi: 10.1111/jdv.12224

28. Bojar RA, Holland KT. Acne and propionibacterium acnes. Clin Dermatol. (2004) 22:375-9. doi: 10.1016/j.clindermatol.2004.03.005

29. Megyeri K, Orosz L, Bolla S, Erdei L, Rázga Z, Seprényi G, et al. Propionibacterium acnes induces autophagy in keratinocytes: involvement of multiple mechanisms. J Invest Dermatol. (2017) 138:750-9. doi: 10.1016/j.jid.2017.11.018

30. O'Rielly DD, Rahman P. Genetics of psoriatic arthritis. Best Pract Res Clin Rheumatol. (2014) 28:673-85. doi: 10.1016/j.berh.2014.10.010

31. Gurevich I, Zhang C, Francis N, Aneskievich BJ. TNIP1, a retinoic acid receptor corepressor and A20-binding Inhibitor of NF- $\mathrm{B}$, distributes to both nuclear and cytoplasmic locations. J Histochem Cytochem. (2011) 59:1101-12. doi: 10.1369/0022155411427728

32. Fitz-Gibbon S, Tomida S, Chiu BH, Nguyen L, Du C, Liu M, et al. Propionibacterium acnes strain populations in the human skin microbiome associated with acne. J Invest Dermatol. (2013) 133:2152-60. doi: 10.1038/jid.2013.21

33. Verstrepen L, Carpentier I, Verhelst K, Beyaert R. ABINs: A20 binding inhibitors of NF-кB and apoptosis signaling. Biochem Pharmacol. (2009) 78:105-14. doi: 10.1016/j.bcp.2009.02.009
34. Lai T-Y, Wu S-D, Tsai M-H, Chuang EY, Chuang L-L, Hsu L$\mathrm{C}$, et al. Transcription of tnfaip3 is regulated by NF- $\kappa \mathrm{B}$ and $\mathrm{p} 38$ via C/EBP $\beta$ in activated macrophages. PLoS ONE (2013) 8:e73153. doi: 10.1371/journal.pone.0073153

35. Heyninck K, De Valck D, Vanden Berghe W, Van Criekinge W, Contreras $\mathrm{R}$, Fiers W, et al. The zinc finger protein A20 inhibits TNF-induced NF- $\kappa \mathrm{B}-$ dependent gene expression by interfering with an RIP- or TRAF2-mediated transactivation signal and directly binds to a novel NF- $\kappa \mathrm{B}$-inhibiting protein ABIN. J Cell Biol. (1999) 145:1471-82. doi: 10.1083/jcb.145.7.1471

36. Oshima S, Turer EE, Callahan JA, Chai S, Advincula R, Barrera J, et al. ABIN-1 is a ubiquitin sensor that restricts cell death and sustains embryonic development. Nature (2009) 457:906-9. doi: 10.1038/nature07575

37. Ramirez VP, Gurevich I, Aneskievich BJ. Emerging roles for TNIP1 in regulating post-receptor signaling. Cytokine Growth Factor Rev. (2012) 23:109-18. doi: 10.1016/j.cytogfr.2012.04.002

38. Nanda SK, Venigalla RKC, Ordureau A, Patterson-Kane JC, Powell DW, Toth RC. et al. Polyubiquitin binding to ABIN1 is required to prevent autoimmunity. J Exp Med. (2011) 208:1215-28. doi: 10.1084/jem.20102177

39. Zhou J, Wu R, High AA, Slaughter CA, Finkelstein D, Rehg JE, et al. A20-binding inhibitor of NF- B (ABIN1) controls Toll-like receptormediated CCAAT/enhancer-binding protein activation and protects from inflammatory disease. Proc Natl Acad Sci USA. (2011) 108:E998-1006. doi: $10.1073 /$ pnas. 1106232108

40. Ganceviciene R, Zouboulis CC. Isotretinoin: state of the art treatment for acne vulgaris. J Dtsch Dermatol Ges. (2010) 8(Suppl. 1):S47-59. doi: 10.1111/j.1610-0387.2009.07238.x

41. Dai X, Yamasaki K, Shirakata Y, Sayama K, Hashimoto K. All-trans-retinoic acid induces interleukin- 8 via the nuclear factor-kappaB and p38 mitogenactivated protein kinase pathways in normal human keratinocytes. J Invest Dermatol. (2004) 123:1078-85. doi: 10.1111/j.0022-202X.2004.23503.x

42. Wojtal KA, Wolfram L, Frey-Wagner I, Lang S, Scharl M, Vavricka SR, et al. The effects of vitamin A on cells of innate immunity in vitro. Toxicol Vitr. (2013) 27:1525-32. doi: 10.1016/j.tiv.2013.03.013

43. Kim SY, Koo JE, Song MR, Lee JY. Retinol suppresses the activation of toll-like receptors in MyD88- and STAT1-independent manners. Inflammation (2013) 36:426-33. doi: 10.1007/s10753-012-9562-2

44. Kelhälä HL, Fyhrquist N, Palatsi R, Lehtimäki S, Väyrynen JP, Kubin ME, et al. Isotretinoin treatment reduces acne lesions but not directly lesional acne inflammation. Exp Dermatol. (2016) 25:477-8. doi: 10.1111/exd.12971

45. Liu PT, Krutzik SR, Kim J, Modlin RL. Cutting Edge: all-trans retinoic acid down-regulates TLR2 expression and function. J Immunol. (2005) 174:246770. doi: 10.4049/jimmunol.174.5.2467

46. Dispenza MC, Wolpert EB, Gilliland KL, Dai JP, Cong Z, Nelson AM, et al. Systemic isotretinoin therapy normalizes exaggerated TLR-2-mediated innate immune responses in acne patients. J Invest Dermatol. (2012) 132:2198-205. doi: 10.1038/jid.2012.111

47. Harant H, De Martin R, Andrew PJ, Foglar E, Dittrich C, Lindley IJ. Synergistic activation of interleukin- 8 gene transcription by all-trans-retinoic acid and tumor necrosis factor-alpha involves the transcription factor NFkappaB. J Biol Chem. (1996) 271:26954-61. doi: 10.1074/jbc.271.43.26954

48. Jugeau S, Tenaud I, Knol AC, Jarrousse V, Quereux G, Khammari A, et al. Induction of toll-like receptors by propionibacterium acnes. $\mathrm{Br} J$ Dermatol. (2005) 153:1105-13. doi: 10.1111/j.1365-2133.2005.06933.x

49. Fathy A, Mohamed RW, Ismael NA, El-Akhras MA. Expression of toll-like receptor 2 on peripheral blood monocytes of patients with inflammatory and noninflammatory acne vulgaris. Egypt J Immunol. (2009) 16:127-34.

Conflict of Interest Statement: The authors declare that the research was conducted in the absence of any commercial or financial relationships that could be construed as a potential conflict of interest.

Copyright (C) 2018 Erdei, Bolla, Bozó, Tax, Urbán, Kemény and Szabó. This is an open-access article distributed under the terms of the Creative Commons Attribution License (CC BY). The use, distribution or reproduction in other forums is permitted, provided the original author(s) and the copyright owner(s) are credited and that the original publication in this journal is cited, in accordance with accepted academic practice. No use, distribution or reproduction is permitted which does not comply with these terms. 


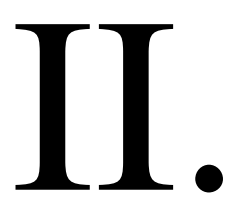




\title{
Factors shaping the composition of the cutaneous microbiota
}

\author{
K. Szabó, ${ }^{1}$ L. Erdei, ${ }^{2}$ B. Sz. Bolla, ${ }^{2}$ G. Tax, ${ }^{2}$ T. Bíró ${ }^{3}$ and L. Kemény ${ }^{1,2}$ \\ ${ }^{1}$ MTA-SZTE Dermatological Research Group, Szeged, Hungary \\ ${ }^{2}$ Department of Dermatology and Allergology, University of Szeged, Szeged, Hungary \\ ${ }^{3}$ DE-MTA 'Lendület' Cellular Physiology Research Group, Departments of Physiology and Immunology, Faculty of Medicine, University of Debrecen, Debrecen, Hungary
}

Linked Comment: Totté and Pasmans. Br J Dermatol 2017; 176:289.

\section{Summary}

\section{Correspondence \\ Kornelia Szabó. \\ E-mail: szabo.kornelia@med.u-szeged.hu}

\section{Accepted for publication}

3 July 2016

\section{Funding sources}

This study was funded by the Hungarian Scientific Research Fund (OTKA NK105369) and the János Bolyai Research Scholarship from the Hungarian Academy of Sciences (K.S). The funders had no role in the study design, data collection, data analysis, manuscript preparation and/or publication decisions.

\section{Conflicts of interest \\ None declared.}

DOI 10.1111/bjd.14967
From birth, we are constantly exposed to bacteria, fungi and viruses, some of which are capable of transiently or permanently inhabiting our different body parts as our microbiota. The majority of our microbial interactions occur during and after birth, and several different factors, including age, sex, genetic constitution, environmental conditions and lifestyle, have been suggested to shape the composition of this microbial community. Propionibacterium acnes is one of the most dominant lipophilic microbes of the postadolescent, sebum-rich human skin regions. Currently, the role of this bacterium in the pathogenesis of the most common inflammatory skin disease, acne vulgaris, is a topic of intense scientific debate. Recent results suggest that Westernization strongly increases the dominance of the Propionibacterium genus in human skin compared with natural populations living more traditional lifestyles. According to the disappearing microbiota hypothesis proposed by Martin Blaser, such alterations in the composition of our microbiota are the possible consequences of socioeconomic and lifestyle changes occurring after the industrial revolution. Evanescence of species that are important elements of the human ecosystem might lead to the overgrowth and subsequent dominance of others because of the lack of ecological competition. Such changes can disturb the fine-tuned balance of the human body and, accordingly, our microbes developed through a long co-evolutionary process. These processes might lead to the transformation of a seemingly harmless species into an opportunistic pathogen through bacterial dysbiosis. This might have happened in the case of P. acnes in acne pathogenesis.

\section{What's already known about this topic?}

- Microbes are integral components of the human ecosystem.

- The cutaneous microbiota plays an important role in the regulation of skin homeostasis

- The composition of skin microbiota is influenced by many factors.

\section{What does this study add?}

- The dominance of Propionibacterium acnes in postadolescent sebum-rich skin regions and its role in acne pathogenesis may be explained by the disappearing microbiota hypothesis.
Our microbiota is the result of constant exposure to bacteria, fungi and viruses, which transiently or permanently inhabit our body parts. Many of these microbial species are not simply passive bystanders, but, together with various human cells, form a complex ecosystem. ${ }^{1-3}$
The first identified human-associated bacterium, Escherichia coli, was isolated from stool samples of healthy and diseased children by paediatrician Theodor Escherich in the $1880 \mathrm{s.}^{4}$ His contemporary, Louis Pasteur, had already hypothesized that normal human flora were essential for life (reviewed by 
Mackowiak). ${ }^{5}$ The idea that microbes can act as important integral components of the human body received particular attention a decade ago; subsequently, studies on microbial communities inhabiting various organs have become increasingly popular. The Human Microbiome Project (HMP) was launched in 2007 to identify and characterize these microorganisms. ${ }^{6}$ Many interesting data have been gathered by internationally coordinated research efforts in the last few years; however, we are still far from completely understanding the exact role of these microbes.

\section{The microbiota}

Various parts of the human body provide appropriate environments for colonization by several microbial species, even in healthy individuals. Surfaces that come into direct contact with the external environment provide constant temperature, moisture and nutrient availability, allowing bacterial and fungal species to be selected through a long co-evolutionary process. ${ }^{7,8}$ Colonization of our skin, regions of the alimentary canal and parts of our urogenital tract has long been known. However, organs previously considered sterile, such as lung and placenta, may also have their own resident community. ${ }^{9-11}$

The relationship between resident microbial communities and human cells is very complex. Previously, it was thought that resident microbes inhabit the available niches and use the nutrients that are present without pathogenesis. Today, it is clear that these species and their metabolic products also play important roles in a wide range of biological functions. In fact, they may regulate the development of cellular and histological features of colonized human organs and help to maintain their proper functions. ${ }^{12-14}$

\section{Factors shaping the composition of the skin microbiota}

The cutaneous microbiota populates the epidermis and the pilosebaceous unit of human skin. ${ }^{15,16}$ To date, approximately 1000 bacterial species belonging to 19 phyla, as well as fungal (dermatophytes) and viral species, have been identified as members of this community. ${ }^{15}$ Actinobacteria (Propionibacterium and Corynebacterium species), Proteobacteria, Firmicutes (Staphylococcus species) and Bacteroidetes are the most common representatives of the four dominant bacterium phyla. Many factors (individual, lifestyle, environmental) influence the microbial diversity of our skin, and changes in any of these conditions can result in rapid alterations of the species composition within the community. ${ }^{15,17,18}$

\section{Early colonization}

Cutaneous colonization generally starts at birth. Individual differences in the composition of the microbiota of the gut and possibly of the skin may be caused by the mode and manner of birth (vaginal or caesarean delivery, hospital or home setting, use of antibiotics, etc.). ${ }^{19-22}$ During vaginal delivery, babies come into contact with their mothers' vaginal microbes, and this encounter will determine the composition of the pioneer colonizers, including Lactobacillus, Prevotella, Atopobium and Snethia species. Children delivered by caesarean section acquire their first inhabitants from their environment (mainly Staphylococcus species and other skin bacteria), which do not necessarily originate from their mothers. Initially, the flora of a newborn is completely identical and undifferentiated at various anatomical sites (e.g. gut, mouth, skin), regardless of the mode of delivery. ${ }^{20}$

Early colonization is a critical event and may have longterm consequences, as microbes that most efficiently adapt to an environment will subsequently become dominant. When two species cannot coexist and compete for the same resources, even a slight advantage (e.g. faster growth, more effective use of the available nutrients or more efficient binding to the available attachment sites of the surrounding tissues) may allow one species to out-compete the other. In ecology, this phenomenon is referred to as competitive exclusion. ${ }^{23}$ In cases of less severe competition, marked delays in the colonization of beneficial species may occur: babies delivered via caesarean section exhibit delayed colonization of Lactobacillus, Bifidobacterium and Bacterioidetes species in the gut. ${ }^{24,25}$ Dominant microbes of a community actively modify the properties of their environment and, as a result, the microbial ecology. Many commensals secrete factors, such as phenol-soluble modulins and bacteriocins from S. epidermidis and acnecin from Propionibacterium acnes, that are bacteriostatic or antibacterial for other species. ${ }^{26-28}$ Early colonizers may also change their microenvironment to enhance their own growth and inhibit the growth of other microbes.

Another factor that may also influence early colonization is the presence of vernix caseosa (VC) on newborn skin. This white, creamy substance is synthesized during the third trimester of neonates. ${ }^{29,30}$ It is highly cellular: polygonal, waterfilled corneocytes are embedded in an amorphous, lipid-rich material. The structure is somewhat similar to the cornified envelope layer called stratum corneum (SC) of postnatal skin, although corneocytes are not interconnected by desmosomal cellular contacts and the lipid matrix does not possess a lamellar architecture. As a result, $\mathrm{VC}$ is considered a mobile, fluidic SC. ${ }^{31,32}$

$\mathrm{VC}$ is mainly composed of water (81\%), lipids (9\%) and proteins $(10 \%) .^{33}$ Its lipid content is mostly of sebaceous origin, synthesized from the third trimester onward, marking an important step in neonatal epidermal barrier maturation. Other important components are proteins, many of which exhibit antimicrobial properties. As a result of its complex composition and structure, VC exhibits multifaceted biological functions. It acts as a mechanical barrier, offers lubrication during birth, has important waterproofing properties and may aid thermoregulation after delivery. Because of its viscous and hydrophobic nature and its protein constituents, VC also has important antimicrobial functions, protecting the baby from the colonization and growth of pathogenic microbes. ${ }^{34,35}$ The 
$\mathrm{pH}$ of the skin surface is 6.0 at birth and becomes slightly more acidic $(\mathrm{pH} 5 \cdot 1)$ during the first 6 weeks of life. VC appears to facilitate these events, further favouring the early colonization of skin commensal microbes as opposed to pathogens. ${ }^{36,37}$ It also provides an antioxidant shield and aids wound healing of newborn skin. Overall, because of its complex functions, $\mathrm{VC}$ is an important substance providing a smooth transition between intra- and extrauterine life, ${ }^{31}$ and aiding the formation of a balanced, human-microbial ecosystem.

Changes in the pattern of early colonization may lead to unfavourable consequences. Early stimuli can critically affect the developing immune system of the baby, and might lead to the development of atopic, chronic inflammatory and allergic diseases later. These effects are well studied in the case of the gut microbiota, but little is known about the exact nature and effect of the cutaneous microbiota on the pathogenesis of such disease. $^{38}$

\section{Host factors}

After early colonization and stabilization on the skin, Streptococcaceae and other Firmicutes, Bacteroidetes, $\beta$ - and $\gamma$-Proteobacteria dominate the microbiome of children. The composition of this community changes during puberty, when endocrine-induced events result in hyperplasia of sebaceous glands and subsequently enhance sebum excretion. ${ }^{30,39}$ The most pronounced alterations affect areas where the density of sebaceous glands is the highest (face, shoulders, chest and back). These locations likely experience the largest shift in composition of the resident microbes. By analysing the composition of microbial communities, investigators have noted that the species diversity clearly decreases with sexual maturation and that lipophilic microbes, including members of the Corynebacteriaceae and Propionibacteriaceae families, gain dominance on the face. ${ }^{39,40}$

Propionibacterium acnes, one of the most dominant lipophilic microbes of human postadolescent skin, is a Gram-positive, anaerobic-fermenting, rod-shaped bacterium. It has been shown to secrete various enzymes, including lipases, that generate fatty acids from sebum lipids, and might compromise the growth of other microbes. ${ }^{28,34}$ Propionibacterium acnes also secretes short-chain fatty acids (SCFAs) during anaerobic fermentation, one of which, propionic acid, clearly exhibits antibacterial effects. ${ }^{41-43}$ The generated free fatty acids, together with the secreted SCFAs, may contribute to the maintenance of a skin $\mathrm{pH}$ that is acidic enough to restrict many microbes. ${ }^{44}$ These data together explain why this bacterium is dominant in sebum-rich skin. The generation of an environment that is hostile to other microbes suggests that the observed decrease of microbiota diversity during puberty is a direct consequence of P. acnes expansion.

After this transitory period, the core composition of the cutaneous microflora stabilizes by early adulthood. The type and number of bacterial groups that become accustomed to our body is intriguingly limited, suggesting the presence of strong selective forces and coevolution. ${ }^{45}$ However, marked differences in microbiome composition can be detected in samples originating from different anatomical locations of the same individual, suggesting that physiological properties of a given niche lead to site-specific differences in the local composition. ${ }^{15,39,46}$ According to Grice and colleagues, ${ }^{15,17}$ the dominant phyla in sebum-rich regions are the Actinobacteria (Propionibacterium species) and Firmicutes (Staphylococcus species). Moist areas (e.g. armpit, interdigital areas, inguinal crease) are mostly populated by Corynebacteria and Staphylococcus. Dry regions (forearm, buttock) host the most diverse, mixed population of Actinobacteria, Proteobacteria, Firmicutes and Bacteriodetes. $^{15,17}$

Sex also has a noteworthy impact on the microflora. Sexspecific differences likely manifest directly after birth, as recent data suggest that the lipid composition of VC in newborn males and females differs: the VC of females seems to contain a higher proportion of wax esters and triacylglycerols with longer hydrocarbon chains than those found in the VC of males. ${ }^{47}$ Fine anatomical and physiological properties of the skin (thickness, $\mathrm{pH}$, composition and rate of sebum secretion, cosmetic use) might also contribute to sex-specific differences, even when comparing the same regions. ${ }^{18,46,48}$ Skin-surface $\mathrm{pH}$ is generally lower and sebum secretion higher in males vs. females in age-matched cohorts. ${ }^{49}$

The reproductive organs also host a specialized microbiome, owing to their large differences in anatomical and physiological properties (e.g. chemical composition, pH). Specific species that normally populate the genitals have been shown to spread to other anatomical regions; thus, bacteria previously characterized as genital (Lactobacillus and Gardnerella in females, Corynebacterium in males) have also been detected in samples originating from other areas, such as the upper buttock. ${ }^{50}$

\section{Geographical, environmental, socioeconomic and lifestyle factors}

Individual habits, together with the properties of the surrounding environment, are important determinants for the composition of human skin microbiota. Westernized lifestyles have clearly reduced the microbial load and diversity in our environment. Epidemiological studies at the end of the twentieth century revealed that changes occurring after the industrial revolution, including personal and household hygiene, as well as declining family size, led to enormous increases in the prevalence of atopic and other diseases. In 1989, David P. Strachan proposed the hygiene hypothesis, ${ }^{51}$ which suggests that the quantity and diversity of the environmental microbes with which we come into contact are crucial for the development of our immune systems. In addition to the effects of contact with environmental microbes, microbial components of the human ecosystem have also been suggested to play important roles in the maintenance of our healthy and balanced states. ${ }^{52,53}$ Important questions remain as to whether and how all these changes can affect our body and homeostasis. 
Agricultural development, urbanization, the Industrial Revolution and Westernization represent prominent shifts in human cultural development that have resulted in changes in individual lifestyles and, most probably, in our microbiota. Analysis of these events is rather difficult, as archival or archaeological materials preserving ancient microbiomes are not readily available. A recent study of historic samples excavated from a monastery in Germany examined dental tissues of human skeletons (c. 950-1200 CE) exhibiting signs of periodontal disease. Results suggests that currently known oral pathogens (e.g. Tannerella forsythia, Porphyromonas gingivalis, Treponema denticola) have long been associated with the development of periodontal disease, regardless of changes in diet and personal oral hygiene, ${ }^{54}$ and might have become part of the human oral flora in parallel with the introduction of farming in the early Neolithic period. ${ }^{55}$ It is also intriguing that these bacteria included sequences similar to antibiotic-resistance genes, long before antibiotics were available. The presence of such sequences were also identified in another study conducted on members of a contemporary population (Yanomami) in the Amazonian jungle, in Venezuela, who live a seminomadic, hunter-gatherer lifestyle that is presumably very similar to the lifestyle of our ancestors. These individuals have been secluded from the Westernized lifestyle and, as reported, have not been exposed to medical doses of antibiotics throughout their history. ${ }^{56}$ However, antibiotic resistance gene-like sequences are present in their microbial genomes, suggesting that our microbiome may have been serving as a reservoir and source of antibiotic resistance. ${ }^{54,56}$

To model changes in the cutaneous microflora throughout human history, several groups now focus on the analysis of contemporary populations with traditional, less industrialized lifestyles. Comparing these groups with Westernized populations might elucidate conditions that can be associated with historical lifestyles. ${ }^{56,57}$ Strikingly, the results from these studies also indicate that more traditional living conditions mostly correlate with higher microbiome diversity. ${ }^{54,56,58}$ The most complex composition reported to date was discovered on Yamomami individuals, except for the oral samples, where species diversity was comparable with North American individuals living a Westernized lifestyle. ${ }^{56,57}$

The cutaneous microbiota of these individuals appears to be highly complex, but Staphylococcus, Propionibacterium, Corynebacterium and Neisseria species are much less dominant for Yamomami and South American Amerindian individuals than for Westernized populations. ${ }^{56,58}$ Generally, Westernization seems to be associated with an increasing dominance of the Actinobacteria phylum and, particularly, the Propionibacterium genus on the skin. ${ }^{58}$

These data corroborate nicely with the disappearing microbiota hypothesis proposed by Martin Blaser. According to this proposition, the two major routes of acquiring resident microflora are (i) vertical acquisition (by maternal transmission to the offspring) and (ii) horizontal transfer (from the surroundings through contaminated environment, food, drinking water and physical contact). Because of changes in hygiene, housing and family models, the latter route has gradually become less prominent in the human population. As a consequence, loss of particular microbes in the maternal generation could be inherited by subsequent generations, and thus the loss could become permanent. The net effect would be gradually decreasing variability in our resident microbes, or, in other words, disappearing microbiota. ${ }^{45,59}$ The combined effects of decreasing diversity for environmental microbes (hygiene hypothesis) and drastic changes in human ecology might also lead to declining diversity in our resident flora (disappearing microbiota hypothesis). These events might be linked to the gradual increase in the prevalence of Westernization diseases, including atopic diseases, such as asthma, as well as obesity and metabolic syndrome. ${ }^{45,59,60}$

Clearly, socioeconomic changes and the resulting lifestyle differences have a great impact on our microbiota; however, it is less clear how seasonal changes, climate and ethnicity affect our cutaneous microbial community. Most of the currently available studies are associated with the HMP and have been conducted in Western countries. ${ }^{61}$ Only a handful of reports have investigated populations living under different climatic zones in different geographical areas. ${ }^{6,57,58,62}$ According to the available data, while the core composition of the microbiome is similar for different populations (Proteobacteria, Firmicutes, Actinobacteria), clear differences have been detected. Relative abundances of various genera can be diverse - perhaps even unique - and population-specific microbes have been identified, such as the Enhydrobacter genus in the cutaneous samples of Chinese individuals. ${ }^{62}$ It should be noted that dissimilarities among populations may not be entirely caused by geographical differences. The lifestyle and socioeconomic differences described above should also be taken into consideration when comparing geographically distinct populations. It is difficult to assess how substantial the impact of environmental differences on the skin microbiome composition is, as such a comparison should include, for example, equally modernized populations exhibiting a very similar lifestyle. One investigation compared groups living in two states within the U.S.A.: Colorado and New York. Even though socioeconomic differences were likely to be small, subtle alterations in the microbiome composition were apparent, suggesting that geographical and climatic factors may also have some effect. ${ }^{58}$

Nonetheless, these studies clearly indicate the importance of well-designed and large-scale investigation of different populations to further increase our understanding of pan-microbiome composition.

\section{What can we learn about the role of the cutaneous microbiota in acne pathogenesis?}

A balanced interaction between microbial and human cells is important for the maintenance and promotion of healthy functions. ${ }^{12,13,50}$ Microbes can synthesize and release nutrients from our food for use by human cells, protect us from the colonization of pathogenic or harmful invaders, beneficially modulate our immune system, and even facilitate differentiation and renewal of certain tissues (e.g. gut 
mucosa). ${ }^{12,63}$ When this delicate and intricate equilibrium is disturbed in our ecosystem, dysbiosis develops, leaving us vulnerable to microbial diseases. Changes in the microenvironment and colonization by an extraneous microbe can contribute to dysbiosis and, together with other pathogenic factors, might lead to diseases such as seborrhoeic dermatitis (Malassezia species), atopic dermatitis (Staphylococcus aureus), postoperative infections (Staphylococcus epidermidis) or acne vulgaris (P. acnes). ${ }^{12,17,64}$

In many cases it is difficult to distinguish clearly between commensal, symbiotic and pathogenic microbes, as the behaviour and impact of a microbe can be strongly contextdependent. ${ }^{45}$ For these reasons, the pathogenic roles of several species, including P. acnes and its involvement in acne vulgaris, are a matter of intense scientific debate. ${ }^{28,65-67}$

Several changes occurring in puberty, such as hormonal changes, androgen excess, sebaceous gland hyperplasia and subsequently enhanced sebum secretion, create a permissive environment for lipophilic bacteria. At this time, a shift from the 'childhood' microbiome, in which Streptococcaceae, Firmicutes, and $\beta$ - and $\gamma$-Proteobacteria predominate, to a more 'mature' composition dominated by Corynebacteriaceae and Propionibacteriaceae occurs. ${ }^{39}$ Changes in the skin microenvironment drive these events, as well as the possible strong competitive exclusion generated by the 'newcomers'. During this transitory period, dysbiosis can occur before the stabilization of the adult ecosystem.

Keeping in mind these natural, developmentally driven changes in microbiome composition, it is interesting to consider the microbial consequences of Westernization. In natural populations, the composition of the cutaneous microflora is more complex and balanced compared with Westernized groups, where P. acnes clearly dominates the postpubertal microbiota. ${ }^{56}$ What causes these differences is currently not known. During puberty, enhanced sebum secretion may provide a growth advantage for the lipophilic P. acnes, and the bacterium can subsequently modify its environment by lowering the $\mathrm{pH}$ as a result of SCFA secretion. In contrast, the disappearing microbiota hypothesis may also provide some explanation. Competing microbes controlling P. acnes growth might have been gradually lost as a result of Westernization. The consequence of such a loss might be the dominance of P. acnes, its enhanced growth and the resulting dysbiosis leading to acne pathogenesis during puberty.

This could also imply that acne is a disease of Westernized populations. Although no reports are available on the incidence of acne in natural populations, earlier reports suggest that acne does not equally affect all populations: acne vulgaris is present in $80-90 \%$ of the adolescents living in developed countries, ${ }^{68,69}$ whereas in isolated communities this ratio can be much lower. Some reports suggest that acne is or was nonexistent in the inhabitants of the island of Okinawa before World War II, the Bantus in South Africa, isolated South American Indians and Pacific Islanders; ${ }^{70-74}$ the lifestyles of all of these populations are considerably less Westernized than those in developed countries. ${ }^{75}$
Whether P. acnes load is higher in the skin of patients with acne than unaffected individuals is currently not clear. Earlier reports provided conflicting results; ${ }^{76-78}$ the reason for this disagreement is likely that the bacterium is located deep in the pilosebaceous units, that it exhibits different culturing properties and that it often presents in a biofilm form. However, it seems that increased incidence of P. acnes biofilms is detectable in the lesional skin samples of patients. ${ }^{79}$ How and why exactly this happens is currently not known. Bacterial quorum sensing could possibly explain this discrepancy: by reaching a threshold density, the bacterium may start to form a biofilm in the pilosebaceous unit and express molecules contributing to bacterial pathogenicity. ${ }^{80}$ Such transformations might lead to dysbiosis and, subsequently, also to acne pathogenesis.

Even if the relative abundance of the bacterium is similar, there are indications that the P. acnes population structure of controls is different for patients with acne. Strains that preferentially present in lesional skin samples might have altered genetic and microbiological properties, as well as pathogenicity. $^{81}$

Although it has not been explicitly proven, we believe that all these data strongly suggest that P. acnes has a role in acne pathogenesis.

\section{How can this knowledge be put into practice?}

Can we somehow overcome the potentially deleterious effects of the disappearance of various species from our microbial ecosystems? Is it possible to modify artificially the pattern of microbial transfer or aid the transfer of a complex, balanced microbiota to prevent various diseases? It may be possible. There are already exploratory clinical studies that are implementing this idea. Alteration of the gut flora by faecal microbiota transplantation has already shown efficacy in severe Clostridium difficile infections and has been proposed for the treatment of other conditions (e.g. inflammatory bowel disease, irritable bowel syndrome, metabolic syndrome), in which the composition of the gut microbiome differs from the healthy state. ${ }^{82}$ Another, widely used method to restore a balanced intestinal microflora is to use probiotics, which are clearly beneficial after antibiotic use. Probiotics might also have beneficial effects for diseases such as obesity, insulin resistance syndrome, type 2 diabetes and nonalcoholic fatty liver disease, although this conclusion requires further, welldesigned, rigorous clinical investigation. ${ }^{83}$

Attempts are also being made to provide an appropriate pioneer flora to babies who are not delivered vaginally. In a recent article, Dominguez-Bello et al. reported a clinical trial to establish a healthy microbiota in babies delivered by caesarean section by wiping them with a gauze previously exposed to the vaginal fluids of their mothers. Although the results are preliminary, the analysed sample size is relatively small and only partial microbiome reconstitution was achieved, the results clearly suggest that during the analysed time period the microbiota of wiped babies delivered by caesarean section are more similar to vaginally delivered children vs. untreated 
neonates delivered by caesarean section. ${ }^{84}$ The consequences of such procedures on the overall health and the prevalence of, for example, atopic and chronic inflammatory diseases is of interest for following in long-term studies.

If the above-proposed model of acne pathogenesis and the role of $\mathrm{P}$. acnes is confirmed, would it be possible to treat this condition by artificially modifying the composition of cutaneous microbiota of the teenage population? Currently, it is difficult to answer this question. Further studies of natural populations living more traditional lifestyles would be very useful to define a core 'ancient' cutaneous microbiota composition. From such knowledge, we could select microbes that might provide an appropriate control over P. acnes dominance but were most likely lost during our socioeconomical development. Topical formulations including these selected species are envisioned for application in a way analogous to probiotic use. Naturally, detailed and rigorous in vitro and in vivo experiments would be needed to test the interaction of the different microbes with one another, their effects on skin cells and, finally, on the whole organism. To support methods for replacing microflora lost by Westernized lifestyles, it will also be necessary to examine lifestyle and other changes that maintain healthy microflora complexity and balance as, presumably, the forces in our modern environments that reduce complexity are still in effect.

\section{Conclusion}

A complex interplay between a host and its microbiota is important for the maintenance of healthy skin function. Because of the polygenic and multifactorial nature of the disease, there are many possible alternative paths leading to the pathogenesis of acne vulgaris. One possible mechanism might be a change in the interaction between the skin cells and the cutaneous microflora leading to an imbalanced state and subsequently causing a 'harmless' commensal, such as P. acnes, to become pathogenic.

\section{Acknowledgments}

We are grateful to the anonymous reviewers for their valuable comments, which greatly improved the final version of the manuscript, and to Shannon Frances for the language correction.

\section{References}

1 Savage DC. Microbial ecology of the gastrointestinal tract. Annu Rev Microbiol 1977; 31:107-33.

2 Ottman N, Smidt H, de Vos WM et al. The function of our microbiota: who is out there and what do they do? Front Cell Infect Microbiol 2012; 2:104.

3 Cho I, Blaser MJ. The human microbiome: at the interface of health and disease. Nat Rev Genet 2012; 13:260-70.

4 Shulman ST, Friedmann HC, Sims RH. Theodor Escherich: the first pediatric infectious diseases physician? Clin Infect Dis 2007; 45:1025-9.
5 Mackowiak PA. The normal microbial flora. N Engl J Med 1982; 307:83-93.

6 Human Microbiome Project Consortium. A framework for human microbiome research. Nature 2012; 486:215-21.

7 Ley RE, Lozupone CA, Hamady $M$ et al. Worlds within worlds: evolution of the vertebrate gut microbiota. Nat Rev Microbiol 2008; 6:776-88.

8 Lee YK, Mazmanian SK. Has the microbiota played a critical role in the evolution of the adaptive immune system? Science 2010; 330: 1768-73.

9 Aagaard K, Ma J, Antony KM et al. The placenta harbors a unique microbiome. Sci Transl Med 2014; 6:237.

10 Marsland BJ, Salami O. Microbiome influences on allergy in mice and humans. Curr Opin Immunol 2015; 36:94-100.

11 Dickson RP, Huffnagle GB. The lung microbiome: new principles for respiratory bacteriology in health and disease. PLoS Pathog 2015; 11:e1004923.

12 Gallo RL, Nakatsuji T. Microbial symbiosis with the innate immune defense system of the skin. J Invest Dermatol 2011; 131:1974-80.

13 Littman DR, Pamer EG. Role of the commensal microbiota in normal and pathogenic host immune responses. Cell Host Microbe 2011; 10:311-23.

14 Kranich J, Maslowski KM, Mackay CR. Commensal flora and the regulation of inflammatory and autoimmune responses. Semin Immunol 2011; 23:139-45.

15 Grice EA, Kong HH, Conlan S et al. Topographical and temporal diversity of the human skin microbiome. Science 2009; 324:11902.

16 Jahns AC, Alexeyev OA. Three dimensional distribution of Propionibacterium acnes biofilms in human skin. Exp Dermatol 2014; 23:687-9.

17 Grice EA, Segre JA. The skin microbiome. Nat Rev Microbiol 2011; 9:244-53.

18 Zeeuwen PL, Boekhorst J, van den Bogaard EH et al. Microbiome dynamics of human epidermis following skin barrier disruption. Genome Biol 2012; 13:R101.

19 Penders J, Thijs C, Vink C et al. Factors influencing the composition of the intestinal microbiota in early infancy. Pediatrics 2006; 118:511-21.

20 Dominguez-Bello MG, Costello EK, Contreras M et al. Delivery mode shapes the acquisition and structure of the initial microbiota across multiple body habitats in newborns. Proc Natl Acad Sci U S A 2010; 107:11971-5.

21 Azad MB, Konya T, Maughan $\mathrm{H}$ et al. Gut microbiota of healthy Canadian infants: profiles by mode of delivery and infant diet at 4 months. CMAJ 2013; 185:385-94.

22 Coughlin CC, Taieb A. Evolving concepts of neonatal skin. Pediatr Dermatol 2014; 31 (Suppl. 1):5-8.

23 Hardin G. The competitive exclusion principle. Science 1960; 131:1292-7.

24 Adlerberth I, Lindberg E, Aberg $\mathrm{N}$ et al. Reduced enterobacterial and increased staphylococcal colonization of the infantile bowel: an effect of hygienic lifestyle? Pediatr Res 2006; 59:96-101.

25 Malago JJ. Contribution of microbiota to the intestinal physicochemical barrier. Benef Microbes 2015; 6:295-311.

26 Fujimura S, Nakamura T. Purification and properties of a bacteriocin-like substance (acnecin) of oral Propionibacterium acnes. Antimicrob Agents Chemother 1978; 14:893-8.

27 Hassan M, Kjos M, Nes IF et al. Natural antimicrobial peptides from bacteria: characteristics and potential applications to fight against antibiotic resistance. J Appl Microbiol 2012; 113:723-36.

28 Christensen GJ, Bruggemann H. Bacterial skin commensals and their role as host guardians. Benef Microbes 2014; 5:201-15. 
29 Pochi PE, Strauss JS, Downing DT. Age-related changes in sebaceous gland activity. J Invest Dermatol 1979; 73:108-11.

30 Zouboulis CC, Boschnakow A. Chronological ageing and photoageing of the human sebaceous gland. Clin Exp Dermatol 2001; 26:600-7.

31 Hoath SB, Pickens WL, Visscher MO. The biology of vernix caseosa. Int J Cosmet Sci 2006; 28:319-33.

32 Rissmann R, Groenink HW, Weerheim AM et al. New insights into ultrastructure, lipid composition and organization of vernix caseosa. J Invest Dermatol 2006; 126:1823-33.

33 Hoeger PH, Schreiner V, Klaassen IA et al. Epidermal barrier lipids in human vernix caseosa: corresponding ceramide pattern in vernix and fetal skin. Br J Dermatol 2002; 146:194-201.

34 Drake DR, Brogden KA, Dawson DV et al. Thematic review series: skin lipids. Antimicrobial lipids at the skin surface. J Lipid Res 2008; 49:4-11.

35 Singh G, Archana G. Unraveling the mystery of vernix caseosa. Indian J Dermatol 2008; 53:54-60.

36 Visscher MO, Narendran V, Pickens WL et al. Vernix caseosa in neonatal adaptation. J Perinatol 2005; 25:440-6.

37 Tollin M, Jagerbrink T, Haraldsson A et al. Proteome analysis of vernix caseosa. Pediatr Res 2006; 60:430-4.

38 Bendtsen KM, Fisker L, Hansen AK et al. The influence of the young microbiome on inflammatory diseases-Lessons from animal studies. Birth Defects Res C Embryo Today 2015; 105:278-95.

39 Oh J, Conlan S, Polley EC et al. Shifts in human skin and nares microbiota of healthy children and adults. Genome Med 2012; 4:77.

40 Trivedi B. Microbiome: the surface brigade. Nature 2012; 492:S60-1.

41 Shu M, Wang Y, Yu J et al. Fermentation of Propionibacterium acnes, a commensal bacterium in the human skin microbiome, as skin probiotics against methicillin-resistant Staphylococcus aureus. PLoS ONE 2013; 8:e55380.

42 Tan J, McKenzie C, Potamitis M et al. The role of short-chain fatty acids in health and disease. Adv Immunol 2014; 121:91-119.

43 Wang Y, Dai A, Huang S et al. Propionic acid and its esterified derivative suppress the growth of methicillin-resistant Staphylococcus aureus USA300. Benef Microbes 2014; 5:161-8.

44 Elias PM. The skin barrier as an innate immune element. Semin Immunopathol 2007; 29:3-14.

45 Blaser MJ, Falkow S. What are the consequences of the disappearing human microbiota? Nat Rev Microbiol 2009; 7:887-94.

46 Fierer N, Hamady M, Lauber CL et al. The influence of sex, handedness, and washing on the diversity of hand surface bacteria. Proc Natl Acad Sci U S A 2008; 105:17994-9.

47 Mikova R, Vrkoslav V, Hanus R et al. Newborn boys and girls differ in the lipid composition of vernix caseosa. PLoS ONE 2014; 9: e99173.

48 Staudinger T, Pipal A, Redl B. Molecular analysis of the prevalent microbiota of human male and female forehead skin compared to forearm skin and the influence of make-up. J Appl Microbiol 2011; 110:1381-9.

49 Man MQ, Xin SJ, Song SP et al. Variation of skin surface $\mathrm{pH}$, sebum content and stratum corneum hydration with age and gender in a large Chinese population. Skin Pharmacol Physiol 2009; 22:190-9.

50 Zeeuwen PL, Kleerebezem M, Timmerman HM et al. Microbiome and skin diseases. Curr Opin Allergy Clin Immunol 2013; 13:514-20.

51 Strachan DP. Hay fever, hygiene, and household size. BMJ 1989; 299: 1259-60.

52 Cookson WO, Moffatt MF. Asthma: an epidemic in the absence of infection? Science 1997; 275:41-2.

53 Heederik D, von Mutius E. Does diversity of environmental microbial exposure matter for the occurrence of allergy and asthma? J Allergy Clin Immunol 2012; 130:44-50.
54 Warinner C, Rodrigues JF, Vyas R et al. Pathogens and host immunity in the ancient human oral cavity. Nat Genet 2014; 46:336-44.

55 Adler CJ, Dobney K, Weyrich LS et al. Sequencing ancient calcified dental plaque shows changes in oral microbiota with dietary shifts of the Neolithic and Industrial revolutions. Nat Genet 2013; 45:450-5.

56 Clemente JC, Pehrsson EC, Blaser MJ et al. The microbiome of uncontacted Amerindians. Sci Adv 2015; 1:pii:e1500183.

57 Yatsunenko T, Rey FE, Manary MJ et al. Human gut microbiome viewed across age and geography. Nature 2012; 486:222-7.

58 Blaser MJ, Dominguez-Bello MG, Contreras M et al. Distinct cutaneous bacterial assemblages in a sampling of South American Amerindians and US residents. ISME J 2013; 7:85-95.

59 Blaser MJ. Who are we? Indigenous microbes and the ecology of human diseases. EMBO Rep 2006; 7:956-60.

60 Blaser MJ. Disappearing microbiota: helicobacter pylori protection against esophageal adenocarcinoma. Cancer Prev Res (Phila) 2008; 1:308-11.

61 Peterson J, Garges S, Giovanni M et al. The NIH Human Microbiome Project. Genome Res 2009; 19:2317-23.

62 Leung MH, Wilkins D, Lee PK. Insights into the pan-microbiome: skin microbial communities of Chinese individuals differ from other racial groups. Sci Rep 2015; 5:11845.

63 Lai Y, Di NA, Nakatsuji T et al. Commensal bacteria regulate Tolllike receptor 3-dependent inflammation after skin injury. Nat Med 2009; 15:1377-82.

64 Bojar RA, Holland KT. Acne and Propionibacterium acnes. Clin Dermatol 2004; 22:375-9.

65 Dessinioti C, Katsambas AD. The role of Propionibacterium acnes in acne pathogenesis: facts and controversies. Clin Dermatol 2010; 28:2-7.

66 Shaheen B, Gonzalez M. A microbial aetiology of acne: what is the evidence? Br J Dermatol 2011; 165:474-85.

67 Williams HC, Dellavalle RP, Garner S. Acne vulgaris. Lancet 2012; 379:361-72.

68 Ghodsi SZ, Orawa H, Zouboulis CC. Prevalence, severity, and severity risk factors of acne in high school pupils: a communitybased study. J Invest Dermatol 2009; 129:2136-41.

69 Saitta P, Keehan P, Yousif J et al. An update on the presence of psychiatric comorbidities in acne patients, part 1: overview of prevalence. Cutis 2011; 88:33-40.

70 Steiner PE. Necropsies on Okinawans; anatomic and pathologic observations. Arch Pathol (Chic) 1946; 42:359-80.

71 Findlay GH. The age incidence of common skin diseases in the white population of the Transvaal. Br J Dermatol 1967; 79:538-42.

72 Park RG. The age distribution of common skin disorders in the Bantu of Pretoria, Transvaal. Br J Dermatol 1968; 80:758-61.

73 Freyre EA, Rebaza RM, Sami DA et al. The prevalence of facial acne in Peruvian adolescents and its relation to their ethnicity. J Adolesc Health 1998; 22:480-4.

74 Cordain L, Lindeberg S, Hurtado $\mathrm{M}$ et al. Acne vulgaris: a disease of Western civilization. Arch Dermatol 2002; 138:1584-90.

75 Szabo K, Kemeny L. Studying the genetic predisposing factors in the pathogenesis of acne vulgaris. Hum Immunol 2011; 72:766-73.

76 Cove JH, Cunliffe WJ, Holland KT. Acne vulgaris: is the bacterial population size significant? Br J Dermatol 1980; 102:277-80.

77 Leyden JJ, McGinley KJ, Vowels B. Propionibacterium acnes colonization in acne and nonacne. Dermatology 1998; 196:55-8.

78 Shaheen B, Gonzalez M. Acne sans P. acnes. J Eur Acad Dermatol Venereol 2013; 27:1-10.

79 Jahns AC, Lundskog B, Ganceviciene R et al. An increased incidence of Propionibacterium acnes biofilms in acne vulgaris: a case-control study. Br J Dermatol 2012; 167:50-8. 
80 Coenye T, Peeters E, Nelis HJ. Biofilm formation by Propionibacterium acnes is associated with increased resistance to antimicrobial agents and increased production of putative virulence factors. Res Microbiol 2007; 158:386-92.

81 Fitz-Gibbon S, Tomida S, Chiu BH et al. Propionibacterium acnes strain populations in the human skin microbiome associated with acne. J Invest Dermatol 2013; 133:2152-60.

82 Kelly CR, Kahn S, Kashyap P et al. Update on fecal microbiota transplantation 2015: indications, methodologies, mechanisms, and outlook. Gastroenterology 2015; 149:223-37.
83 Saez-Lara MJ, Robles-Sanchez C, Ruiz-Ojeda FJ et al. Effects of probiotics and synbiotics on obesity, insulin resistance syndrome, type 2 diabetes and non-alcoholic fatty liver disease: a review of human clinical trials. Int J Mol Sci 2016; 17:E928.

84 Dominguez-Bello MG, De Jesus-Laboy KM, Shen N et al. Partial restoration of the microbiota of cesarean-born infants via vaginal microbial transfer. Nat Med 2016; 22:250-3. 


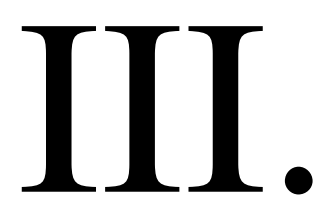


Accepted for publication in Acta Dermato-Venereologica

"Published in Acta Dermato-Venereologica by Society for Publication of Acta DermatoVenereologica" https://www.medicaljournals.se/acta/content/abstract/10.2340/00015555-3707

Title: Tumour Necrosis Factor Alpha-induced Protein 3 Negatively Regulates Cutibacterium acnes-induced Innate Immune Events in Epidermal Keratinocytes

Running head: TNFAIP3 controls microbiome-induced inflammation in human keratinocytes

Lilla Erdei ${ }^{1,2}$, Beáta Szilvia Bolla ${ }^{1,2}$, Renáta Bozó ${ }^{2}$, Gábor Tax ${ }^{2}$, Edit Urbán ${ }^{3}$, Katalin Burián $^{4}$, Lajos Kemény ${ }^{1,2,5,{ }^{*}}$ and Kornélia Szabó ${ }^{2,5, *}$

1. HCEMM-SZTE Skin Research Group, Szeged, Hungary

2. Department of Dermatology and Allergology, University of Szeged, Szeged, Hungary

3. Department of Public Health, University of Szeged, Szeged, Hungary

4. Institute of Clinical Microbiology, University of Szeged, Szeged, Hungary

5. MTA-SZTE Dermatological Research Group, Szeged, Hungary

* These two authors contributed equally to this work.

\section{Correspondence:}

Kornélia Szabó

szabo.kornelia@med.u-szeged.hu 


\begin{abstract}
(max. 150 words)
Human epidermal keratinocytes sense the presence of human skin microbiota through pathogen recognition receptors, such as toll-like receptors (TLRs), and induce innate immune and inflammatory events. Inflammation is absent in healthy epidermis, despite the continuous presence of cutaneous microbes, evidence of effective immune regulatory mechanisms. We investigated TNFAIP3, a negative regulator of TLR and NF- $\kappa B$ signaling pathways, and its role in these regulatory events. We found that a broad spectrum of TLR ligands induced TNFAIP3 expression, as did live Cutibacterium acnes, which is involved in acne pathogenesis. Changes in bacterium-induced, dose-dependent TNFAIP3 expression were JNK- and NF- $\mathrm{KB}$-dependent and resulted in altered cytokine and chemokine levels in in vitro cultured human keratinocytes. In acne lesions, TNFAIP3 mRNA expression was elevated compared to non-lesional skin samples from the same individuals. Our finding suggests that TNFAIP3 may have a general role in fine regulation of microbiota-induced cutaneous immune homeostasis.
\end{abstract}

\title{
Keywords
}

TNFAIP3, microbiota, Cutibacterium acnes, acne vulgaris, innate immunity 


\section{INTRODUCTION}

The human skin separates our body from the external environment and plays a role in the formation of a complex physical, chemical and immunological barrier (1). The skin also provides a natural habitat for the cutaneous microbiota, a collection of microbial species living in close contact with the human cells (2). Human epidermal keratinocytes are the major cellular components of the skin and are in direct contact with these microbes.

Keratinocytes are immunocompetent cells able to sense different external danger signals, known as pattern- and danger-associated molecular patterns (PAMPs and DAMPs), through their pattern recognition receptors (PRRs), such as Toll-like receptors (TLRs). PAMPs and DAMPs originate from human and microbial cells and induce TLR activation, as well as subsequent activation of innate immune and inflammation responses in keratinocytes $(3,4)$. In past decades, research efforts identified endogenous negative regulators of these signaling pathways that are able to control these processes. One such molecule is the TNF alpha induced protein 3 (TNFAIP3), also known as A20, which is a ubiquitin-editing enzyme involved in the inhibition of nuclear factor kappa B (NF- $\mathrm{KB})$ activation and tumor necrosis factor $(\mathrm{TNF} \alpha)$ induced apoptosis (5).

In healthy skin, the presence of the cutaneous microbiota does not lead to excessive immune activation, even though the microbes express various PAMP molecules and are continuously present on the skin. Meanwhile, human cells must retain their capacity to respond to pathogenic microorganisms, necessitating complex regulation to maintain healthy body functions and homeostasis. Currently, little is known about the exact mechanisms by which keratinocytes are able to distinguish between pathogens and harmless members of the microbiota, tolerate the presence of residential microbes, or control the immune and inflammatory events that they are able to induce.

Cutibacterium acnes (C. acnes, formerly known as Propionibacterium acnes, $P$. acnes) is a commensal bacterium, which colonizes the upper part of the epidermis and the pilosebaceous units, where it becomes a dominant species during puberty $(6,7)$. Human epidermal keratinocytes recognize this bacterium via TLR2 and TLR4 $(8,9)$. Receptor activation leads to the induction of multiple signaling paths, including the NF- $\kappa \mathrm{B}$ and mitogen activated protein kinase (MAPK) pathways, resulting in the upregulation of several inflammatory mediators, which in turn promote local inflammation (10-12). Although immune events induced by commensal microbes are well characterized, especially for $C$. acnes, much less is known about the factors or signaling pathways that contribute to the negative regulation of these processes. Such processes are necessary to protect the host from the deleterious consequences of unnecessary inflammation. In a search of such factors, a recent study showed the potential role of TNFAIP3 in immune events induced by Staphylococcus epidermidis (S. epidermidis) in keratinocytes (13). Based on these data, we aimed to determine whether the described negative regulatory function of TNFAIP3 is specific for processes induced by $S$. epidermidis or TNFAIP3 has a more general role in controlling different TLR ligand-induced signaling pathways in keratinocytes. We also examined whether TNFAIP3 contributes to the control of other cutaneous microbes by assessing its role in $C$. acnes-induced inflammation.

In our study, we found that multiple TLRs $(1 / 2,2,3,4,5$ and 2/6) are able to regulate TNFAIP3 expression, suggesting a general role for this molecule in innate immune and inflammatory cascades. Apart from the applied microbial ligands, TNFAIP3 also appeared to play negative regulatory roles in inflammation induced by live $C$. acnes bacterium in keratinocytes. This pathway regulated the mRNA and protein expression of TNFAIP3, and the 
extent of regulation was positively correlated with the dose of $C$. acnes applied to the keratinocytes. For proper control in keratinocytes, the correct function of Jun kinase (JNK) and $\mathrm{NF}-\kappa \mathrm{B}$ signaling pathways are essential. In addition, TNFAIP3 levels affected basal NF- $\mathrm{B}$ activities and basal and C. acnes-induced cytokine and chemokine levels.

These results suggest a general, negative regulatory role of TNFAIP3 in TLR-induced signaling cascades in keratinocytes and, via these pathways, the maintenance of healthy interactions between with our microbes in the human ecosystem.

\section{MATERIALS AND METHODS}

\section{Cell cultures and models}

The human immortalized keratinocyte cell line HPV-KER was used for our experiments. For the generation of the HPV-KER cell line, normal human adult keratinocytes (NHEK) were obtained from a healthy individual undergoing routine plastic surgery at our department and transfected with the pCMV vector containing the HPV16/E6 oncogene. A stable cell line was established by continuous culturing over 70 passages before the start of our experiments. Previously, we have shown, that HPV-KER cells respond to TLR stimuli, (C. acnes, poly (I:C), poly (dA:dT)) similarly to that of NHEK cells (14-17).

Cells were cultured in keratinocyte serum-free medium (KSFM, Life Technologies, Carlsbad, USA) containing 1\% antibiotic/antimycotic (AB/AM) solution, containing penicillin, streptomycin, amphotericin B (Sigma Aldrich, St. Louis, MO, USA) and supplemented with epidermal growth factor and brain pituitary extract under standard laboratory conditions $\left(37^{\circ} \mathrm{C}\right.$ in a humidified atmosphere containing $5 \% \mathrm{v} / \mathrm{v} \mathrm{CO}_{2}$ ).

Ex vivo skin biopsies were taken from skin specimens obtained from the Plastic Surgery Unit of our Department. Written informed consent was obtained from investigated individuals. The study was approved by the Human Investigation Review Board of the University of Szeged (PSO-EDAFN-002, 34/2015, 3517, 23 February 2015, Szeged, Hungary) and complied with the ethical standards of research and was in accordance with the Helsinki Declaration. For ex vivo organotypic skin (OS) cultures, $1 \mathrm{~cm} \mathrm{x} 1 \mathrm{~cm}$ biopsies were washed first with normal saline solution (NSS) containing 2\% AB/AM, followed by a wash with AB/AM-free NSS. Subsequently, the biopsies were placed onto the upper chamber of Transwell ${ }^{\circledR}$ Inserts (Corning, New York, USA) and kept at the air-liquid interphase. The dermal part of the biopsies was in contact with DMEM F12 liquid culture medium (Lonza, Basel, Switzerland) supplemented with 10\% FBS (EuroClone, Milan, Italy) lacking AB/AM.

\section{Data analyzes}

TNFAIP3 gene expression was analyzed using publicly available microarray data from the GEO Profile Database (GDS2478 datasets, ID: 33444972), which compares the total RNA content of healthy skin $(n=6)$ with lesional $(n=6)$ and non-lesional $(n=6)$ samples from acne patients (18).

\section{Treatments}

TLR1/2 (Pam3CSK4, $5 \mu \mathrm{g} / \mathrm{ml}$ ), TLR2 (peptidoglycan, PGN, $10 \mu \mathrm{g} / \mathrm{ml}$ ), TLR3 (poly I:C, 20 $\mu \mathrm{g} / \mathrm{ml}$ ), TLR4 (lipopolysaccharide, LPS, $2.5 \mu \mathrm{g} / \mathrm{ml}$ ), TLR5 (flagellin, FLA, $0.5 \mu \mathrm{g} / \mathrm{ml}$ ) and 
TLR2/6 (FSL-1, $1 \mu \mathrm{g} / \mathrm{ml}$ ) ligands were applied to HPV-KER cells for 3, 6, 12 and 24 hours (all reagents from InvivoGen, Toulouse, France).

For bacterial treatment, HPV-KER cells were plated in AB/AM-free KSFM culture medium and co-cultured with live $C$. acnes strains belonging to different phylogenetic groups within the species (889, I/A, ATCC 11828, II, 6609, I/B) at various multiplicity of infection (MOI). Ex vivo skin models were treated with the $C$. acnes 889 strain at a density of $3 \times 10^{7}$ colony forming units per $\mathrm{cm}^{2}$ for 24 hours. $C$. acnes strains were cultured and stored as previously described in detail (19).

The selective inhibitors of JNK (sp 600125, $10 \mu \mathrm{M}$ ), NF- $\kappa B$ (Bay 11-7085, $10 \mu \mathrm{M}$ ), p38 (sb 203580, $10 \mu \mathrm{M}$ ), ERK1/2 (PD 098059, $20 \mu \mathrm{M}$ ), or, as a control, DMSO were applied to the cells for 1 hour (all reagents from Sigma Aldrich, St. Louis, MO, USA).

\section{Transfection, plasmids and siRNA-mediated gene silencing}

For transient siRNA-mediated gene silencing, siRNA was delivered by the Santa Cruz siRNA Transfection Reagent (Santa Cruz Biotechnology, Texas, USA) according to the manufacturer's instructions. ON-TARGETplus SMARTpool TNFAIP3 siRNA or ONTARGETplus Non-targeting Pool (Dharmacon, Lafayette, USA) constructs were used at $25 \mathrm{nM}$ concentration.

NF- $\kappa B$ luciferase reporter assay was performed using the PathDetect pNF- $\kappa B-L u c$ CisReporter Plasmid (Stratagene, California, USA), pGL4.75[hRluc/CMV] vector, which was transfected with X-tremeGENE 9 DNA Transfection Reagent (Roche, Indiana, USA). For analysis, the Firefly \& Renilla Dual Luciferase Assay Kit (Biotium Inc, California, USA) was used according to the manufacturer's instructions.

\section{ELISA}

C. acnes-treated and control HPV-KER cell culture supernatants were collected and levels of secreted IL-8, IL-6 and CCL5 were measured by enzyme-linked immunosorbent assay (ELISA, PeproTech EC Ltd., London, UK) according to the manufactures' instructions.

\section{RNA isolation, cDNA synthesis and real-time RT-PCR}

Total RNA from HPV-KER cells or the epidermal part of OS cultures were isolated using TRI-Reagent (Molecular Research Center; Cincinnati, USA). cDNA synthesis was performed using $1 \mu \mathrm{g}$ RNA with the iScript TM cDNA Synthesis kit (Bio-Rad, Hercules, USA). Changes in mRNA expression were detected by real-time RT-PCR using the Universal Probe Library (Roche, Indiana, USA) or the TaqMan Gene Expression Assay (Thermo Scientific, Rockford, USA). Supplementary Table 1 lists the PCR protocols and primer sequences used. All data were normalized to the $18 \mathrm{~S}$ rRNA using the $\Delta \Delta \mathrm{C}_{\mathrm{t}}$ method and compared to the time-matched untreated control samples.

\section{Protein isolation and western blot analysis}

For the preparation of whole cell lysates, samples were collected and lysed in lysis buffer containing $20 \mathrm{mM}$ 4-(2-hydroxyethyl)-1-piperazineethanesulfonic acid, $150 \mathrm{mM} \mathrm{KCl}, 1 \mathrm{mM}$ $\mathrm{MgCl}_{2}, 1 \mathrm{mM}$ DTT, 5\% Triton X-100, 10\% glycerol, 0.1\% NP-40, 1\% Protease Inhibitor Cocktail, phenylmethylsulfonyl fluoride and 0.5\% SDS (all from Sigma Aldrich, St. Louis, MO, USA). Protein concentrations were measured with the BCA Protein Assay Kit (Thermo Scientific, Rockford, USA). Samples $(30 \mu \mathrm{g})$ were separated on a 7.5\% SDS-PAGE gel and 
transferred to nitrocellulose membrane (Bio-Rad, Hercules, USA), blocked in Tris-buffered saline containing 5\% non-fat dried milk. Membranes were incubated overnight at $4{ }^{\circ} \mathrm{C}$ with primary anti-TNFAIP3 antibody diluted 1:1000 (Abcam, Cambridge, United Kingdom) and anti-actin (Sigma Aldrich, St. Louis, MO, USA) diluted 1:1000. Subsequently, membranes were incubated for 1 hour at room temperature with a horseradish peroxidase-conjugated antirabbit IgG secondary antibody (Santa Cruz Biotechnology, Texas, USA) diluted 1:2000. Proteins were visualized with luminol (Bio-Rad, Hercules, USA and Advansta Inc., CA, USA) using an Omega Lum ${ }^{\mathrm{TM}} \mathrm{G}$ Imaging System (Gel Company, CA, USA) or C-digit blot scanner (LI-COR Biosciences, Lincoln, NE).

\section{Fluorescence microscopic analysis}

Frozen sections of ex vivo skin models were pre-incubated with PBS for 5 minutes, fixed with $4 \%$ paraformaldehyde for 5 minutes and permeabilized with $0.25 \%$ TritonX-100 (Thermo Scientific, Rockford, USA) containing Tris-buffered saline (TBS) for 10 minutes. For blocking, TBS containing 5\% fetal bovine serum (EuroClone, Milan, Italy) and 5\% normal goat serum (Sigma Aldrich, St. Louis, MO, USA) was used for 1 hour at room temperature. Cells were stained for 1 hour with anti-human TNFAIP3 antibody diluted 1:300 or rabbit IgG (Santa Cruz Biotechnology, Texas, USA) for isotype control. As a secondary antibody, Alexa Fluor 546 conjugated anti-rabbit IgG (Thermo Scientific, Rockford, USA) was used for 1 hour at room temperature. Nuclei were stained for 5 minutes with 4',6-diamidino-2-phenylindole (DAPI) diluted 1:100. TNFAIP3 was visualized using a ZEISS LSM 880 Confocal Laser Scanning Microscope (Zeiss, Oberkochen, Germany), magnification: 63x.

\section{Statistical analysis}

Unless otherwise noted, all data are presented as mean \pm standard error of the mean (SEM) of three independent experiments. For real time RT-PCR analyzes and ELISA, each treatment was performed at least in triplicate; for western blot and fluorescence microscopic analysis, each treatment was performed once in every independent experiment. Data were compared using paired, two-sample t-test with Holm-Bonferroni correction using the R software, version 3.6.2 (R-Studio, Boston, MA). A probability value of less than 0.05 was considered significant.

\section{RESULTS}

\section{TNFAIP3 expression changes upon TLR activation in keratinocytes}

Keratinocytes are able to sense different PAMPs and DAMPs with their TLRs. We investigated whether the activation of different TLRs using selected ligands influences TNFAIP3 expression of an in vitro cultured human keratinocyte cell line. For this we treated HPV-KER cells with Pam3CSK4 (TLR1/2), PGN (TLR2), poly I:C (TLR3), LPS (TLR4), FSL1 (TLR5) and FLA (TLR2/6, ) and analyzed TNFAIP3 mRNA and protein expression with realtime RT-PCR and western blot analysis.

We found that TNFAIP3 mRNA expression rapidly increases in response to all the investigated TLR ligands. The intensity and kinetics of these changes varied and depended on the ligand used (Figure 1/A). TNFAIP3 protein expression levels also rapidly increased: we 
detected elevated levels 6 hours after ligand treatment, and these levels remained high after 24 hours (Figure 1/B). These results suggest that TNFAIP3 is a generally induced molecule upon TLR activation in keratinocytes.

\section{C. acnes bacterium increases TNFAIP3 expression in keratinocytes}

To analyze whether $C$. acnes affects TNFAIP3 expression levels in keratinocytes, we cocultured HPV-KER cells with the $C$. acnes 889 bacterium strain (MOI=100) and analyzed the mRNA and protein expression changes with real-time RT-PCR and western blot analysis. We found that TNFAIP3 mRNA levels rapidly and transiently increased in the presence of the bacterium, reaching a maximum 12 hours after bacterial treatment (Figure 2/A).

Various $C$. acnes strains may differentially affect the cellular and molecular properties of human keratinocytes $(16,19,20)$. To analyze possible strain-specific regulation, we co-cultured the HPV-KER cells with different $C$. acnes strains (MOI=100) belonging to various phylogenetic groups within the species (C. acnes 889 - IA, 6609 - IB, ATCC 11828 - II) and compared their effects. We found that all of the used $C$. acnes strains were able to induce TNFAIP3 expression changes in HPV-KER cells. We detected slight differences in the induction rates, but the kinetics of the expression changes appeared similar (Supplementary Figure 1). Based on these observations, we used the C. acnes 889 strain in the subsequent experiments.

TNFAIP3 protein expression levels also rapidly increased in response to the bacterium (MOI=100) (Figure 2/C) and remained high during the time-course of our study.

We also determined whether changes in $C$. acnes-induced TNFAIP3 expression were dosedependent by co-culturing HPV-KER cells with different MOIs. We found that the extent of TNFAIP3 mRNA and protein expression changes depended on the bacterial dose (Figure 2/B, D).

To confirm that the observed changes were not specific properties of the immortalized keratinocyte cell line used, we performed the experiments also with NHEK cells. We found similar TNFAIP3 expression changes upon $C$. acnes treatment as we described in HPV-KER cells. C. acnes treatment resulted in elevated TNFAIP3 mRNA levels and dose-dependent protein expression changes (Supplementary Figure 2).

To further test TNFAIP3 inducibility in keratinocytes in the presence of $C$. acnes, we tested ex vivo organotypic skin (OS) cultures. In this model, normal epithelial tissue architecture is present, and as a result, skin physiology is better preserved. OS cultures were treated with live C. acnes bacterium and changes in the mRNA expression were analyzed using real-time RTPCR. TNFAIP3 mRNA expression levels increased in the epidermis part of the OS cultures upon $C$. acnes treatment, similarly to what we observed in monolayer cultures (Figure $2 / \mathrm{G}$ ).

Earlier studies have already shown that TNFAIP3 protein is expressed in the human epidermis (21). We confirmed this finding by immunofluorescence staining of OS cultures, where the TNFAIP3 protein was present throughout the entire epidermis, especially in the most 
differentiated keratinocyte layers. Immunofluorescence microscopy analysis also revealed elevated TNFAIP3 protein levels in response to C. acnes treatment (Figure $2 / \mathrm{H}$ ).

Changes in C. acnes-induced TNFAIP3 expression are dependent on JNK and NF- $\kappa B$ signaling pathways

We also investigated the signaling mechanisms playing important roles in TNFAIP3 regulation. The dimerization partner, RB-like, E2F and multi-vulval class B (DREAM) transcription complex is known to repress its transcription when bound to the DRE3 and DRE4 elements in the TNFAIP3 promoter region in human lung microvessel endothelial cells (HLMVECs). The TNFAIP3 promoter also harbors functional NF- $\kappa \mathrm{B}$ binding sites $(22,23)$. To identify the possible signaling pathways that are involved in $C$. acnes-induced upregulation of TNFAIP3 in keratinocytes, we investigated the contribution of selected pathways playing important roles in the bacterium-induced signaling events (10-12). We inhibited the NF- $\kappa B$ and MAPK (JNK, p38 and ERK1/2) signaling pathways using specific inhibitors and analyzed changes in TNFAIP3 mRNA and protein expression in HPV-KER cells after co-culturing the cells for 12 and 24 hours with the $C$. acnes 889 strain.

We found that inhibition of the JNK signaling pathway decreased basal TNFAIP3 mRNA expression, whereas $C$. acnes-induced mRNA levels were affected by JNK and NF- $\mathrm{BB}$ inhibition (Figure 2/E). Dual inhibition of these two signaling pathways almost completely prevented the bacterium-induced changes in mRNA expression (Supplementary Figure 3), suggesting that these two pathways play major roles in the transcriptional regulation of the TNFAIP3 locus. We also detected decreased protein levels upon JNK and NF- $\mathrm{BB}$ pathway inhibition in the C. acnes-treated samples (Figure 2/F).

TNFAIP3 affects the basal and C. acnes-induced levels of inflammatory mediators in keratinocytes

To analyze the role of TNFAIP3 in the regulation of key inflammatory mediators, siRNAmediated silencing was performed. Silencing resulted in decreased TNFAIP3 protein levels (Figure 3/A). Next, we analyzed the effect of TNFAIP3 on NF- $\kappa \mathrm{B}$ activity using a luciferase reporter assay. In these samples, the basal NF- $\mathrm{B}$ promoter activity significantly increased, compared to the scrambled-siRNA (scr-siRNA) transfected controls (Figure 3/B).

We also monitored the consequences of these events and found that TNFAIP3 silencing significantly increased the basal mRNA expression of TNF $\alpha$, interleukin (IL-) $1 \alpha$, IL-6, IL-8 and C-C motif chemokine ligand 5 (CCL5) and the secreted levels of IL-6 and IL8, compared to the scr-siRNA control samples. These data suggest that TNFAIP3 may have important roles in the establishment and maintenance of keratinocyte cellular homeostasis by controlling the constitutive expression of different key inflammatory mediators.

We also investigated the effect of TNFAIP3 knockdown on the $C$. acnes-induced levels of IL-6, IL8 and CCL5, and found that silencing also significantly increased the levels of mRNA and secreted protein (Figure 3/C, D).

TNFAIP3 expression differ in acne lesions 
C. acnes may play important roles in the regulation and maintenance of epidermal homeostasis, as well as in acne vulgaris pathogenesis as an opportunistic pathogen because of bacterial dysbiosis. To analyze whether TNFAIP3 tissue levels differ in healthy and lesional skin samples, we analyzed data from a publicly available GEO Profile dataset, comparing the total RNA content of healthy individuals with lesional and non-lesional skin samples of acne patients (18). We found significantly increased levels of TNFAIP3 mRNA expression in the lesional skin samples compared to non-lesional skin of acne patients (Figure 4).

\section{DISCUSSION}

The cutaneous microbiota plays important roles in the maintenance of epidermal homeostasis. It protects against pathogenic microbes, promotes cell differentiation in the host and keeps the immune system in an alert state, ready to mount a quick response to repel pathogenic attacks $(2,7)$. These functions require balanced interactions among the human cells and the microbes. When this balance shifts, microbial dysbiosis may occur, resulting in the pathogenesis that manifests as skin disease, such as Malassezia spp in seborrheic dermatitis, $S$. aureus and S. epidermidis in atopic dermatitis (AD) and C. acnes in acne vulgaris $(24,25)$.

Research efforts in the last few decades clearly indicate that epidermal keratinocytes are immunocompetent cells $(4,26,27)$. They are equipped to recognize external microbes, mount immediate and efficient responses to maintain the integrity of the organism, and alert the immune system of harm. Interestingly, both pathogens and members of the skin microbiota are able to initiate this internal danger program in keratinocytes, and induce innate immune and inflammatory events in these cells through the expression of PRRs, such as TLRs (28). However, these microbes are constantly present in the skin, and, therefore, microbiota-induced immune activations must be tightly regulated to avoid unnecessary inflammation and to maintain skin homeostasis. In past decades, several negative regulators of the TLR and NF- $\mathrm{B}$ signaling pathways have been identified, among these is TNFAIP3 (5).

TNFAIP3, also known as A20, is a ubiquitin editing enzyme. Through its zinc finger domains, ubiquitin ligase and deubiquitinase activity, TNFAIP3 controls TNF Receptors (TNFR) and TLR pathways at different levels (29). TNFAIP3 acts directly on receptor interacting protein (RIP) 1 and NF-Kappa-B Essential Modifier (NEMO), which dampen downstream signaling cascades. TNFAIP3 also controls ubiquitin conjugating enzyme E2 N (UBE2N) and cellular inhibitor of apoptosis proteins 1 and 2 (cIAP1/2) enzymes complexes by destabilizing their interaction. This protein also accelerates the dissociation of linear ubiquitin chain assembly complex (LUBAC) and inhibitory kappa B kinases (IKK) /NEMO, leading to the termination of NF- $\kappa \mathrm{B}$ activation (30).

Tight regulation of TNFAIP3 is important for the maintenance of cellular homeostasis. In a murine model, cell-specific deletion of the TNFAIP3 gene in various cells with important innate and adaptive immune functions resulted in spontaneous development of inflammation $(30,31)$. 
In corroboration with these results, polymorphisms within and near the TNFAIP3 locus have been shown to be associated with different autoimmune and chronic inflammatory diseases, including psoriasis (PSO), systemic lupus erythematosus, multiple sclerosis and rheumatoid arthritis (32-35).

In agreement with other studies (21), we showed that TNFAIP3 is expressed through the entire epidermis, exhibiting higher levels in the more differentiated keratinocyte layers. This gradient may be formed parallel with natural differentiation processes, as one of the major regulators of keratinocyte differentiation is calcium $\left(\mathrm{Ca}^{2+}\right)$, and together with $\mathrm{Ca}^{2+}$-induced differentiation, TNFAIP3 expression also increases in in vitro cultured normal human keratinocytes $(21,36)$. The molecular details of these events are currently not known, but it may be mediated through the DREAM transcription factor, which is $\mathrm{Ca}^{2+}$ regulated and also plays important roles in the regulation of TNFAIP3 transcription $(22,37)$.

To analyze the role of TNFAIP3 in the regulation of innate immune and inflammatory events in keratinocytes, we activated different TLRs (1/2, 2, 3, 4, 5 and 2/6) by challenging HPV-KER cells with well-known PAMPs (Pam3CSK4, PGN, poly I:C, LPS, FLA and FSL-1) and analyzed changes in TNFAIP3 expression. All of the investigated TLR ligands increased both the mRNA and protein expression levels of TNFAIP3 in HPV-KER cells; however, the extent of the changes and the time-kinetics were ligand-dependent. These data are in agreement with previous findings with different cell types, suggesting that TNFAIP3 is a generally expressed, universal regulator of the TLR signaling pathways.

Based on these results, we aimed to investigate whether TNFAIP3 also plays a role in $C$. acnes-induced immune and inflammatory events in human keratinocytes. First, we checked whether the bacterium itself influences TNFAIP3 expression in vitro and ex vivo. We found that both the mRNA and protein expression increased rapidly in a dose-dependent manner in response to C. acnes in cultured HPV-KER cells as well as in the epidermal part of an ex vivo skin model. A similar effect was described recently for another member of the skin microbiota, S. epidermidis (13). These results, taken together, indicate that the TNFAIP3 protein may play important roles in the regulation of immune and inflammation activation in response to various members of the cutaneous microbiota.

C. acnes is one of the most prominent cutaneous microbes in the sebum-rich skin regions from adolescence and plays a role in the pathogenesis of acne vulgaris as a result of microbial dysbiosis (24). We were curious to see whether TNFAIP3 levels differ in control individuals and lesional and non-lesional skin samples from acne patients. To answer this question, we compared publicly available microarray data in the GEO Profile database (ID: 33444972) and found that, in patients, mRNA expression of TNFAIP3 significantly increases in papules compared to the non-lesional skin samples of the same patients. These results suggest that, in the lesional skin, apart from the production of different pro-inflammatory mediators, expression of anti-inflammatory molecules also increases. These latter molecules may play important roles in inflammation control to avoid excessive, potentially tissue-damaging reactions. To determine whether increased TNFAIP3 mRNA levels in the lesional skin corresponds to elevated protein levels, further research is necessary. If mRNA and protein levels correspond, the accumulation 
may be an important step during the healing of lesions and the restoration of epidermal homeostasis.

Interestingly, acne is not the only inflammatory skin disease in which TNFAIP3 levels change. In PSO and AD, studies showed lower TNFAIP3 mRNA levels in lesional skin samples, compared to skin from healthy individuals. Moreover, in PSO patients, TNFAIP3 mRNA and protein levels were lower in lesional skin than in non-lesional samples of the same patients. In contrast, TNFAIP3 mRNA expression levels in atopic dermatitis (AD) patients, did not differ in the involved and non-involved samples $(21,38)$. It is not clear whether this observation represents a true difference in the pathogenesis of these chronic inflammatory skin conditions. One possible explanation is that the levels of negative regulators may rapidly change throughout the different phases of disease pathogenesis, and the above observations represent only temporal variations. It is interesting to note, however, that inability to control innate immune activation due to decreased TNFAIP3 levels may lead to persisting, chronic inflammation, and the net outcome and the severity of inflammation possibly depends on the balance of pro- and anti-inflammatory factors. As a result, this molecule may serve in the future as a drug target for treatment.

Previous studies found differences in $C$. acnes strain composition in healthy and acne skin (39), and various strains may differently affect the cellular and molecular biology functions as well as keratinocyte viability $(16,20,40,41)$. Slight differences were found in the TNFAIP3 mRNA expression levels when the HPV-KER cells were co-cultured with different $C$. acnes strains $(889$, ATCC11828, 6609), but the expression kinetics were very similar. The used $C$. acnes strains differ, among others, in their growth rate, amount of secreted metabolites (e.g., various short-chain fatty acids - SCFAs), and pathogenic factors (e.g Christie-Atkins-MunchPeterson (CAMP) factors). These properties may be responsible for the observed differences $(16,42)$. This is similar to what we reported in our previous work, in which we analyzed a TNFAIP3 interaction partner, TNFAIP3 interacting protein 1 (TNIP1), another negative regulator, using similar experimental conditions (43). These findings suggest that strainspecific biological effects of $C$. acnes are likely not due to the differential induction of various negative regulators upon immune and inflammation induction in keratinocytes.

Multiple signaling pathways and transcriptional factors are involved in the transcriptional regulation of TNFAIP3. In LPS-activated bone-marrow-derived macrophages from mice, NF$\kappa \mathrm{B}$ and $\mathrm{p} 38$ regulate TNFAIP3 expression through its NF- $\mathrm{B}$ and CCAAT-enhancer-binding protein (C/EBP $\beta$ ) binding sites (23). However, in Pam3CSK4-activated THP1 cells, NF- $\mathrm{B}$ inhibition has no effect: GSK3-related pathways regulate changes in TNFAIP3 expression, possibly through the cAMP response element binding protein (CREB) (44). Others also showed that the DREAM complex represses its transcriptional activation through downstreamresponsive elements (DRE3 and DRE4) binding sites in mice and HLMVECs (22). These findings suggest that transcriptional regulation of TNFAIP3 is possibly dependent on the type of cell and stimuli. We found that the basal mRNA expression of TNFAIP3 is regulated by JNK, whereas JNK and NF- $\mathrm{BB}$ signaling pathways are involved in the control of $C$. acnesinduced changes in TNFAIP3 mRNA and protein expression, for which p38 and ERK1/2 inhibition had no effect. Dual inhibition of JNK and NF- $\kappa B$ signaling pathways almost 
completely inhibited $C$. acnes-induced changes in mRNA expression, which suggest that these two signaling pathways have a major impact on the bacterium-induced regulation of TNFAIP3 mRNA levels in HPV-KER cells.

A recent study suggested that TNFAIP3 may help S. epidermidis to persist as a commensal on the human skin, through the control of bacterium-induced NF- $\kappa \mathrm{B}$ activation, IL-1 $\beta$ and human beta-defensin-2 (HBD-2) production in keratinocytes (13). Based on these findings, we aimed to analyze whether this negative regulatory function also occurs in $C$. acnes-induced inflammatory processes. For these experiments, we found that silencing TNFAIP3 significantly increased basal NF- $\mathrm{KB}$ promoter activities, as well as the mRNA expression of proinflammatory cytokines and chemokines (TNF $\alpha$, IL-1 $\alpha$, IL-6, IL-8 and CCL5) in HPV-KER cells. Co-culturing of silenced cells with the $C$. acnes 889 strain also leads to increased TNF $\alpha$, IL-1 $\alpha$, IL-6, IL-8 and CCL5 mRNA expression, as well as elevated IL-8, IL-6 and CCL5 protein secretions. These data argue that TNFAIP3 plays important roles in the regulation of $C$. acnesdriven molecular events in keratinocytes as well as in the maintenance of cellular homeostasis by regulating the cutaneous inflammatory program under homeostatic conditions (45).

TNFAIP3 possibly operates in a negative regulatory cycle in keratinocytes (Figure 5.). In this control loop, TNFAIP3 regulates the signaling consequences of a broad spectrum of TLR ligands, which, in turn, also affects TNFAIP3 levels. This is in agreement with the currently available research data, suggesting TNFAIP3 functions in, for example, poly-I:C-induced inflammatory responses in human keratinocytes, as well as in imiquimod- and acetone-induced PSO and dermatitis in mouse models, respectively $(21,38)$. TNFAIP3 also plays important roles in the induction of Pam3CSK4-tolerance in monocytic THP-1 cells, arguing for a generalized role for this molecule in different TLR-mediated molecular processes (44).

In summary, TNFAIP3 plays important roles in the regulation of TLR-induced signaling pathways in keratinocytes, and, through these roles, contributes to the control of $C$. acnesinduced innate immune and inflammatory events. By fine-tuning the microbiota-induced signaling processes, TNFAIP3 may help to maintain a homeostatic condition between the skin and the cutaneous microflora.

Currently, several different therapeutic modalities are available for the treatment of acne vulgaris. They may be clinically effective, although all of them only treat an existing condition, and most of them have substantial side effects (e.g., antibiotics, retinoids) (46, 47). Targeting negative regulators of the $C$. acnes-induced inflammatory pathways themselves may help to develop novel, well-tolerated therapeutic modalities for the treatment of acne vulgaris. Further studies should evaluate the potential use and efficacy of TNFAIP3 as such a target.

\section{ACKNOWLEDGEMENT}

We wish to thank Tanácsné Bajkán Andrea for her excellent technical assistance and Shannon Frances for language editing. This work was supported by the GINOP-2.3.2-15-201600015 and OTKA NK105369 research grants. The project has received funding from the EU's Horizon 2020 research and innovation program under grant agreement No. 739593. 
KS is a recipient of the János Bolyai Research Scholarship of the Hungarian Academy of Sciences. KS was supported by the UNKP-18-4. EL was supported by the UNKP-18-3 of New National Excellence Program of the Ministry of Human Capacities. KS was supported by the ÚNKP-19-4. EL was supported by the ÚNKP-19-3 of New National Excellence Program of the Ministry for Innovation and Technology. The funders had no role in study design, data collection and analysis, decision to publish or preparation of the manuscript.

The authors declare that the research was conducted in the absence of any commercial or financial relationships that could be construed as a potential conflict of interest. 
Figure 1. mRNA and protein expression of TNFAIP3 increases in response to TLR activation. HPV-KER cells were treated with different TLR ligands (Pam3CSK4, PGN, poly I:C, FLA, FSL-1), and TNFAIP3 mRNA and protein levels were analyzed at the indicated timepoints. A) Changes in TNFAIP3 mRNA expression were analyzed by real-time RT-PCR; data were normalized to $18 \mathrm{~S}$ rRNA using the $\Delta \Delta \mathrm{C}_{t}$ method and compared to the time-match untreated control values. Error bars are SEM. B) Changes in TNFAIP3 protein expression were analyzed by western blot analysis and quantitated using an Image Pro Plus; all data were normalized to actin. Statistical analyzes: paired, two-sample t-test with Holm-Bonferroni correction : * $\mathrm{p}<0.05$. $\wedge$
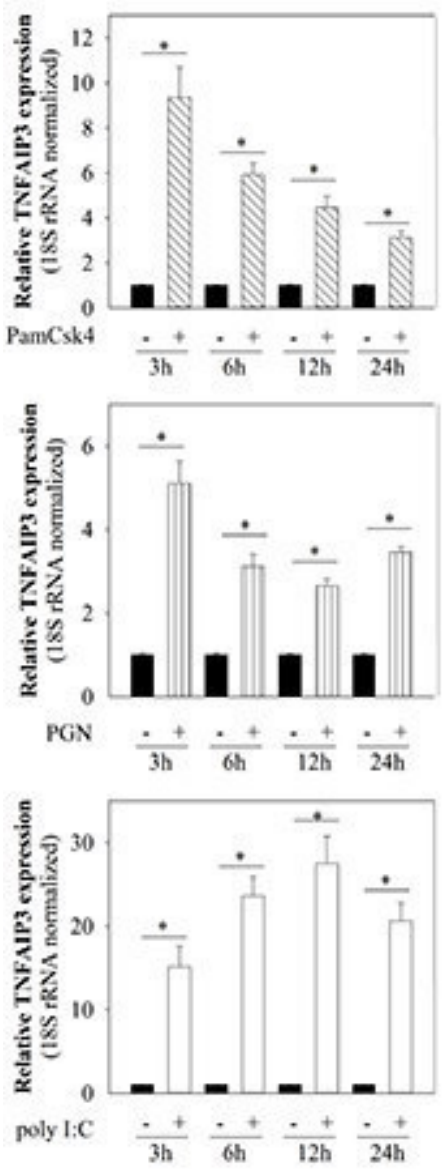
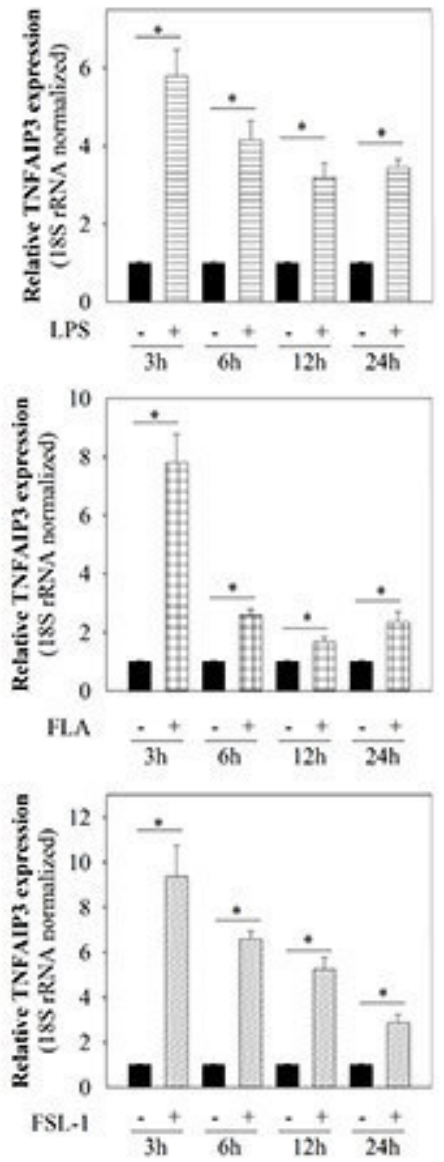
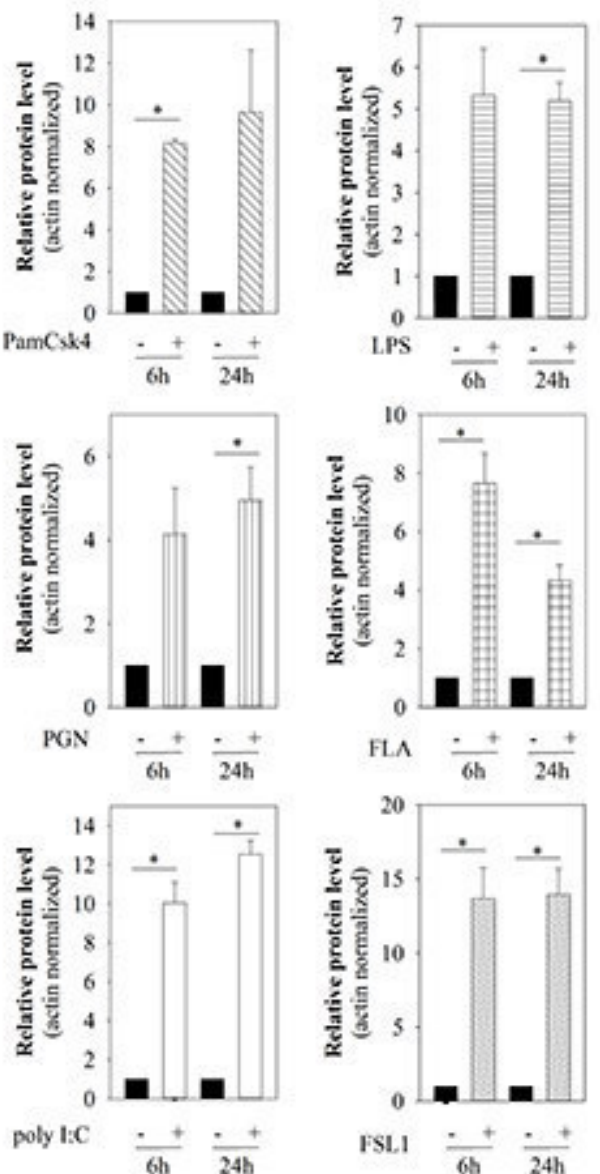

Figure 2. $C$. acnes upregulates the mRNA and protein expression of TNFAIP3. HPV-KER cells $(\mathrm{A}-\mathrm{F})$ and OSs $(\mathrm{G}, \mathrm{H})$ were treated with $C$. acnes, and changes in TNFAIP3 mRNA and protein levels were analyzed. A, B) mRNA expression was analyzed by real-time RT-PCR; data were normalized to $18 \mathrm{~S}$ rRNA and compared to the time-match untreated control values. Error bars are SEM. C, D) TNFAIP3 protein expression was analyzed by western blot and quantified using Image Pro Plus; all data were normalized to actin and compared to the time-match untreated control values. Statistical analyzes: paired, two-sample t-test with Holm-Bonferroni correction : ${ }^{*} \mathrm{p}<0.05$. E, F) Cells were plated and pretreated with selective inhibitors for JNK, $\mathrm{NF}-\kappa \mathrm{B}, \mathrm{p} 38$ and ERK1/2 or, as a control, DMSO for 1 hour. For mRNA expression studies, $C$. acnes challenge was performed for 12 hours and analyzed by real-time RT-PCR. All data were normalized to the $18 \mathrm{~S}$ rRNA and compared to DMSO-treated control samples. For protein expression analyses, cells were treated with $C$. acnes for 24 hours, subjected to western blot analysis and quantified with an Image Pro Plus; all data were normalized to actin and compared to the time-match untreated control values. Statistical analyzes: paired, two-sample t-test with 
Holm-Bonferroni correction, in which basal TNFAIP3 expression, $* \mathrm{p}<0.05$, or C. acnesinduced TNFAIP3 expression, \# $\mathrm{p}<0.05$, were compared. G, H) OS cultures were treated with $3 \times 10^{7}$ bacterium $/ \mathrm{cm}^{2}$ for 24 hours. The epidermis was separated by dispase digestion and the mRNA expression was analyzed only in epidermal cells by real-time RT-PCR. The protein expression was visualized using immunofluorescence staining for TNFAIP3 (green) and DAPI (blue) in the full thickness skin biopsy.
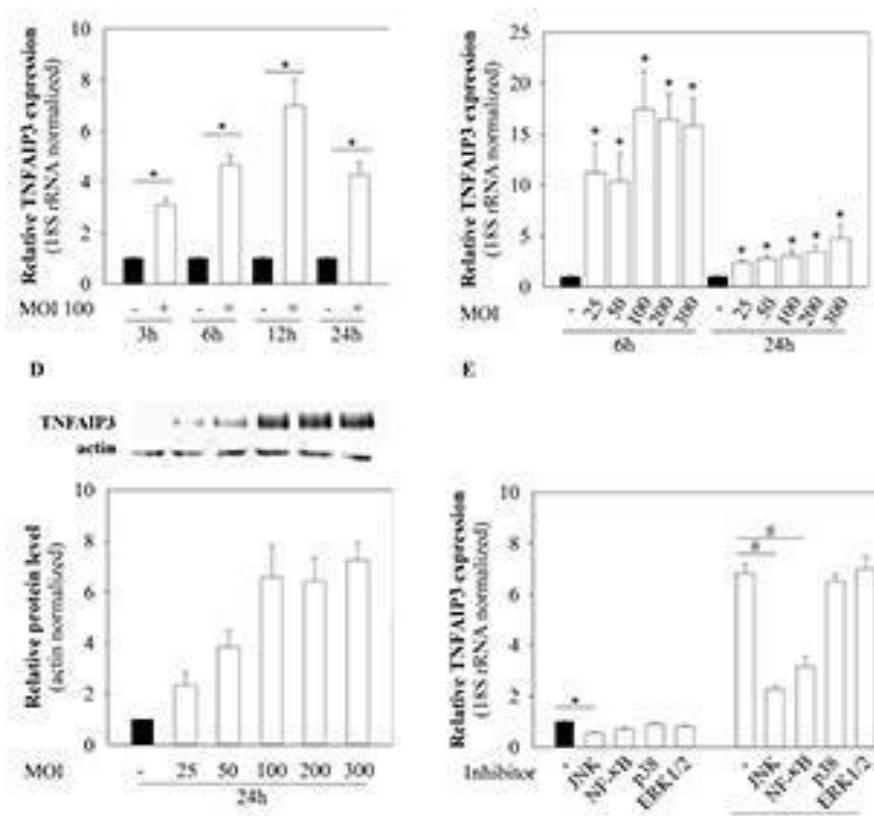

$\varepsilon$
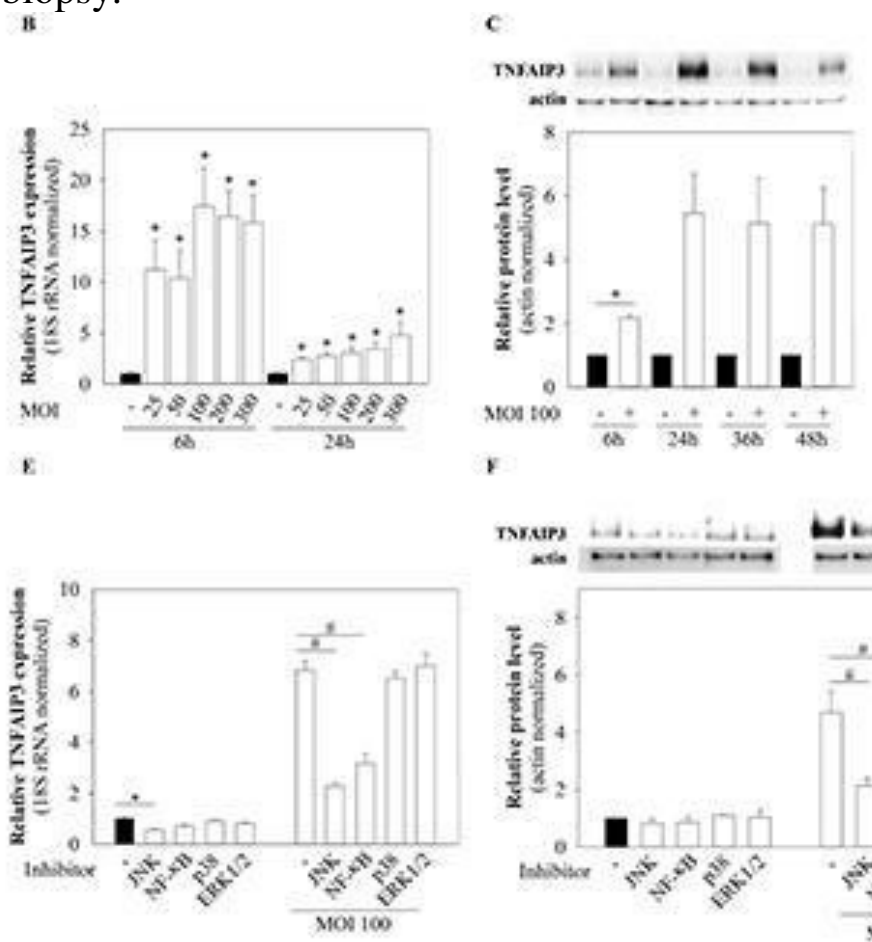

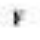

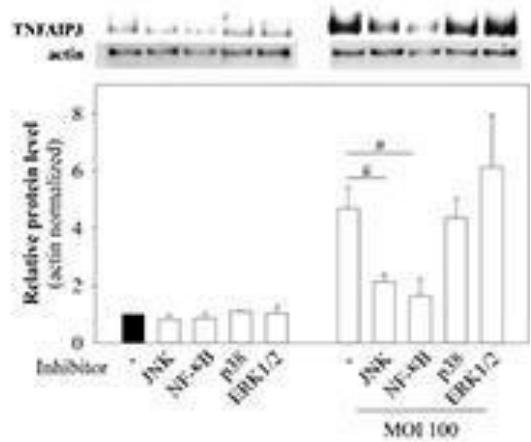

G

11

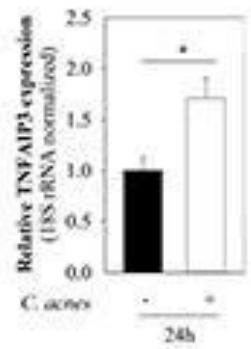

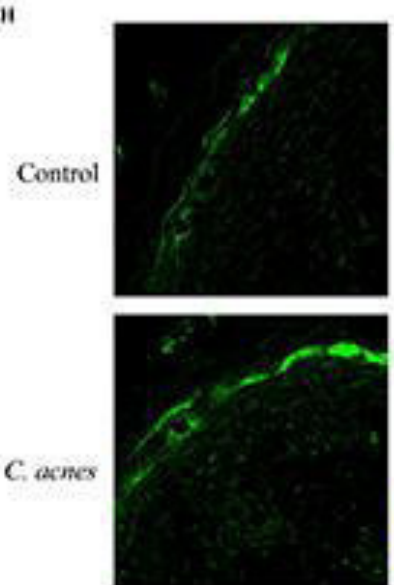

TNFAIP3
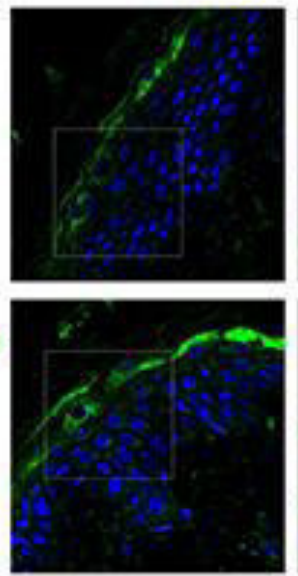

TNFAIP3. DAPI

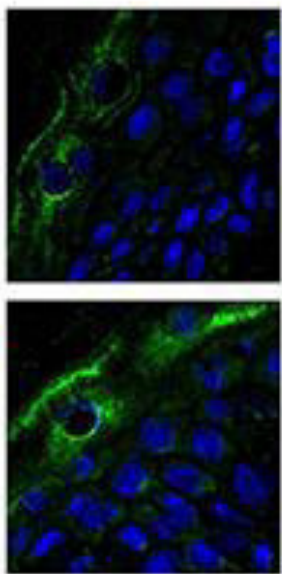

TNFAIP3, DAPI

Figure 3. TNFAIP3 negatively regulates downstream targets of TLR signaling pathways. Transient siRNA-mediated silencing was performed for 48 hours in HPV-KER cells. A) TNFAIP3 protein levels were analyzed by western blot analysis and quantified using Image Pro Plus. All data were normalized to actin. Error bars are SD. B) NF- $\mathrm{BB}$ promoter activity was measured with luciferase reporter assay, for which all data were normalized to Renilla and compared to src-siRNA containing samples. Error bars are SEM. Statistical analysis paired, two sample t-test: $* \mathrm{p}<0.05$. C) After silencing, cells were treated with $C$. acnes for 6 hours and basal and bacterium-induced mRNA expression of TNF $\alpha$, IL- $1 \alpha$, IL- 6 , IL- 8 and CCL5 were monitored by real time RT-PCR. All data were normalized to $18 \mathrm{~S}$ rRNA and compared to 
untreated scr-siRNA containing samples. Error bars are SEM. Statistical analyzes: paired student t-test with Holm-Bonferroni correction, $* \mathrm{p}<0.05$. D) After silencing, cells were treated with $C$. acnes for 24 hours and secreted IL-6, IL-8 and CCL5 proteins were measured by ELISA. Error bars are SEM. Statistical analysis: paired student t-test with Holm-Bonferroni correction, $* \mathrm{p}<0.05$.

$\mathbf{A}$

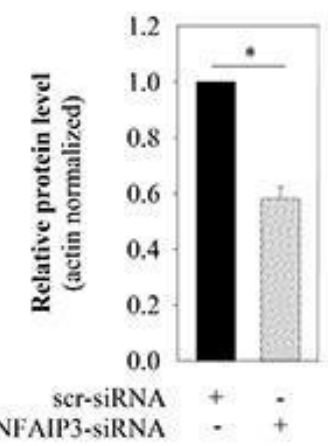

B

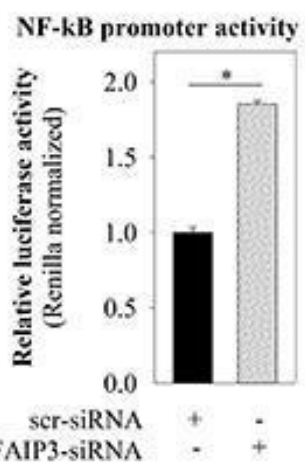

c

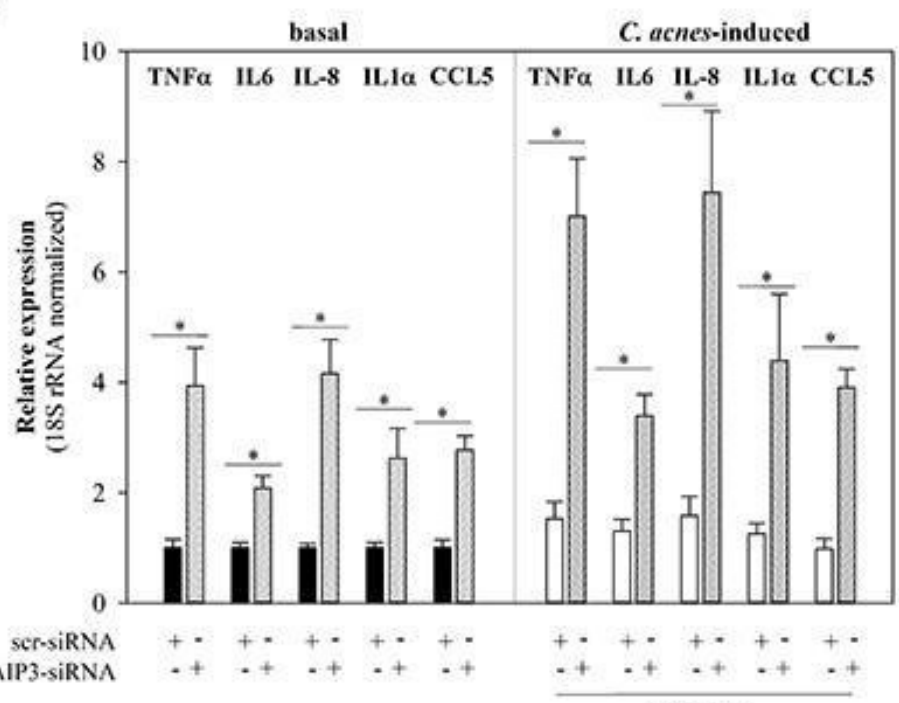

D MOI 100

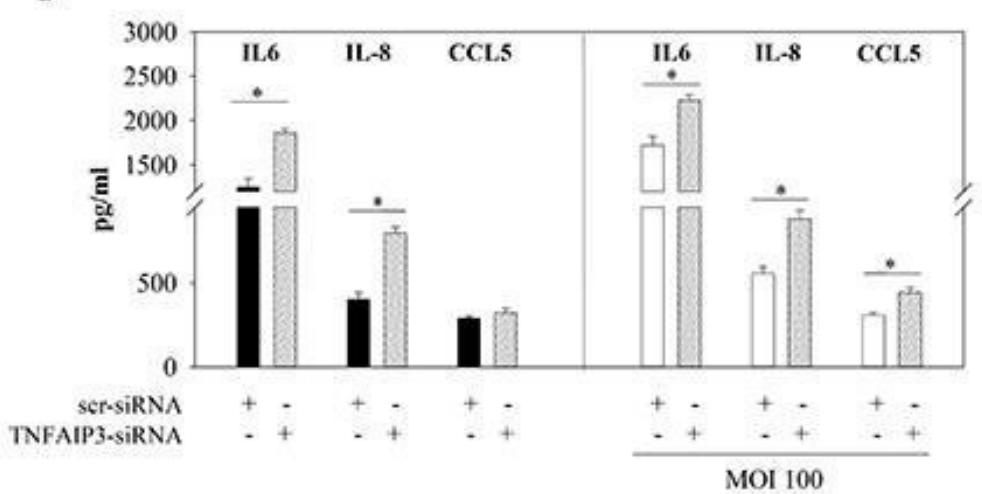

Figure 4. mRNA expression profile of TNFAIP3 in healthy skin and skin from acne patients. TNFAIP3 expression profile from Geo Profile data were analyzed and compared with paired student t-test with Holm-Bonferroni correction, ${ }^{*} \mathrm{p}<0.05$. 


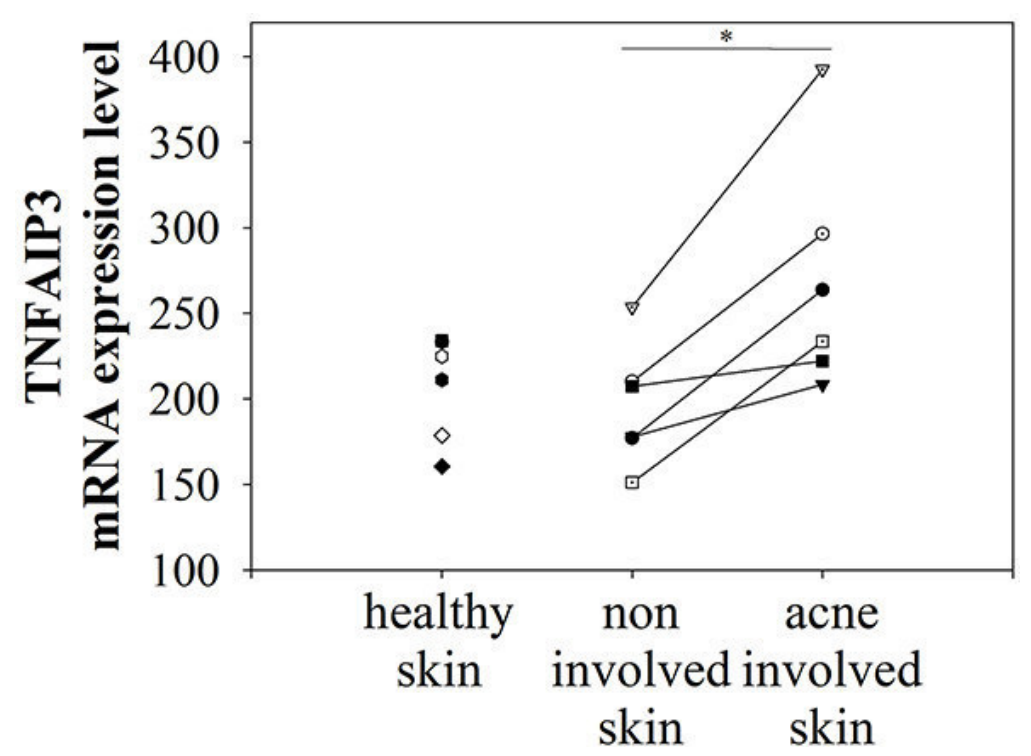

Figure 5. Negative regulation of $C$. acnes-induced TLR signaling events by TNFAIP3 in keratinocytes. $C$. acnes induce TLR 2 and 4 activation in keratinocytes, which leads to the induction of canonical NF- $\kappa \mathrm{B}$ and MAPK-dependent signaling cascades. When NF- $\mathrm{BB}$ is activated, it can regulate the expression changes of TNFAIP3 through binding to special NF$\kappa \mathrm{B}$ binding sites in its promoter region. The generated TNFAIP3 protein in turn can act back and interferes, among them with $\mathrm{NF}-\mathrm{\kappa B}$ activation, forming a negative regulatory feedback loop. Beside NF- $\kappa \mathrm{B}$, JNK is also able to regulate TNFAIP3 expression changes. Created with BioRender.com. 


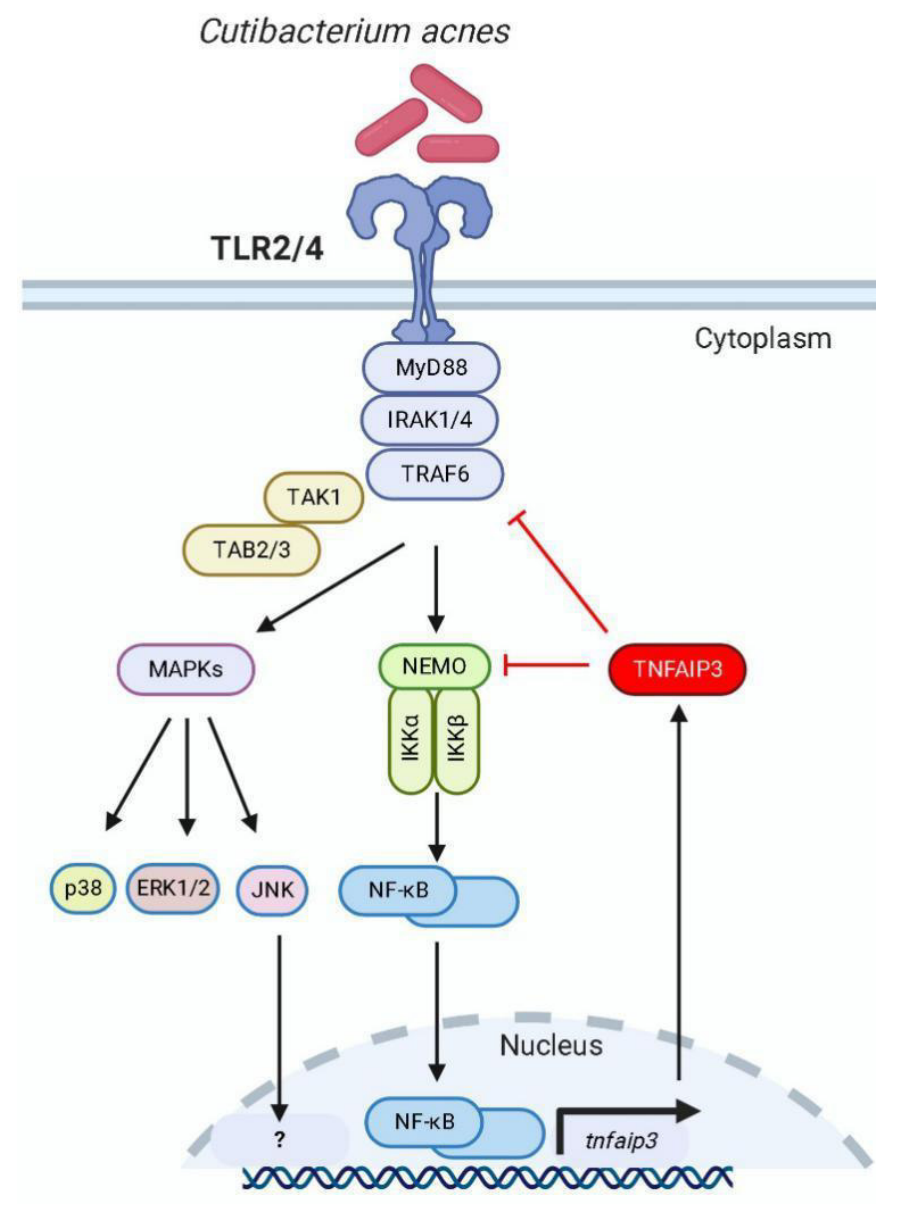

Supplementary table 1. Real-time RT-PCR primers and protocol.

\begin{tabular}{|c|c|c|c|c|c|c|}
\hline \multirow{2}{*}{ Gene } & \multirow{2}{*}{ Primer sequences } & \multirow{2}{*}{ Probe } & \multicolumn{4}{|c|}{ PCR protocol } \\
\hline & & & Denaturation & Annealing & Extension & Cycle \\
\hline 18S rRNA & $\begin{array}{l}\text { FWD: CGCTCCACCAACTAAGAACG } \\
\text { REV: CTCAACACGGGAAACCTCAC }\end{array}$ & 77 & \multirow{7}{*}{$95^{\circ} \mathrm{C}$} & \multirow{5}{*}{$58^{\circ} \mathrm{C}$} & \multirow{7}{*}{$37^{\circ} \mathrm{C}$} & \multirow{7}{*}{$40 \mathrm{x}$} \\
\hline TNFAIP3 & $\begin{array}{l}\text { FWD: TGCACACTGTGTTTCATCGAG } \\
\text { REV: ACGCTGTGGGACTGACTTTC }\end{array}$ & 74 & & & & \\
\hline IL-1 $\alpha$ & $\begin{array}{l}\text { FWD: GGTTGAGTTTAAGCCAATCCA } \\
\text { REV: TGCTGACCTAGGCTTGATGA }\end{array}$ & 6 & & & & \\
\hline IL-6 & $\begin{array}{l}\text { FWD: CAGGAGCCCAGCTATGAACT } \\
\text { REV: GAAGGCAGCAGGCAACAC }\end{array}$ & 45 & & & & \\
\hline CCL5 & $\begin{array}{l}\text { FWD: TGCCCACATCAAGGAGTATTT } \\
\text { REV: CTTTCGGGTGACAAAGACG }\end{array}$ & 59 & & & & \\
\hline TNFA & $\begin{array}{l}\text { Taq Man Genexpression as say } \\
\mathrm{Hs} 00174128 \mathrm{ml}\end{array}$ & & & \multirow{2}{*}{$60^{\circ} \mathrm{C}$} & & \\
\hline IL-8 & $\begin{array}{l}\text { Taq Man Genexpression as say } \\
\text { Hs } 001743103 \text { m } 1\end{array}$ & & & & & \\
\hline
\end{tabular}

Supplementary figure 1. TNFAIP3 expression changes in response to C. acnes ATCC11828 and 6609 bacteria. HPV-KER cells were treated with $C$. acnes and changes in TNFAIP3 mRNA levels were analyzed by real-time RT-PCR (representative experiment). Data were normalized 
to $18 \mathrm{~S}$ rRNA and compared to the time-match untreated control values. Error bars are SD. Statistical analysis: paired, two-sample t-test, $* \mathrm{p}<0.05$.
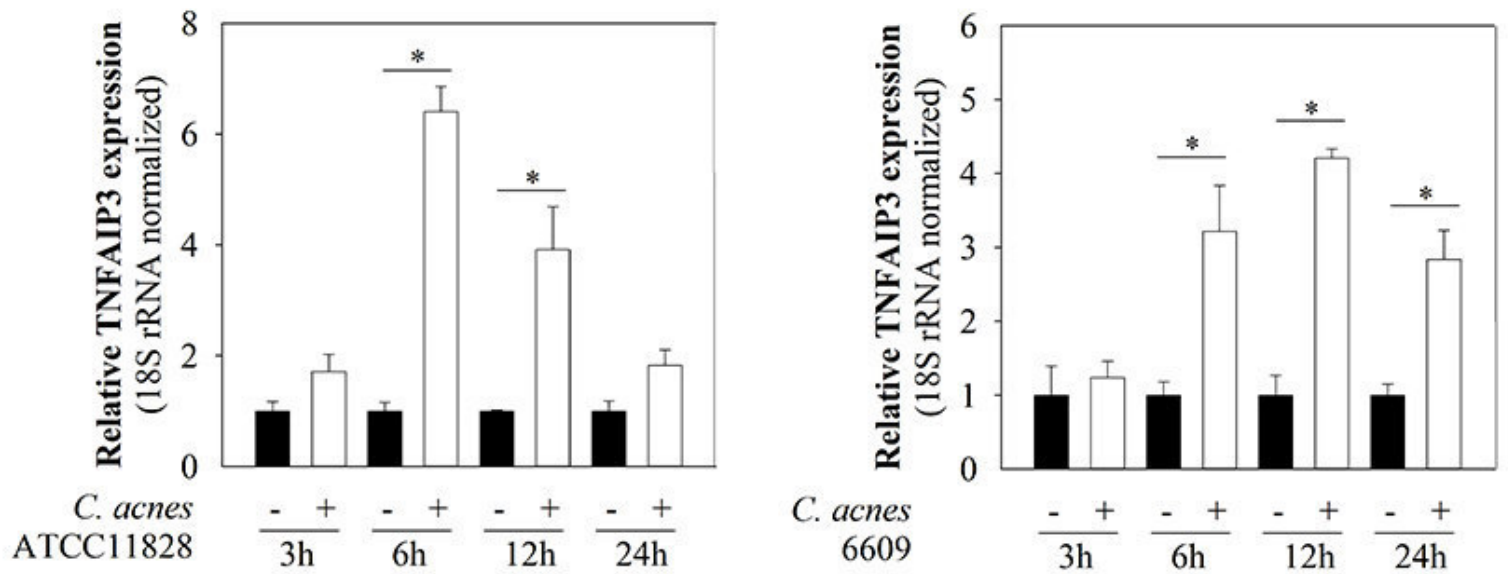

Supplementary figure 2. C. acnes upregulates the mRNA and protein expression of TNFAIP3 in NHEK cells. NHEK cells were treated with $C$. acnes, and changes in TNFAIP3 mRNA and protein levels were analyzed. A) mRNA expression was analyzed by real-time RTPCR; data were normalized to $18 \mathrm{~S}$ rRNA and compared to the time-match untreated control values. B) TNFAIP3 protein expression was analyzed by western blot analysis, where actin served as a loading control. Representative experiments.

A

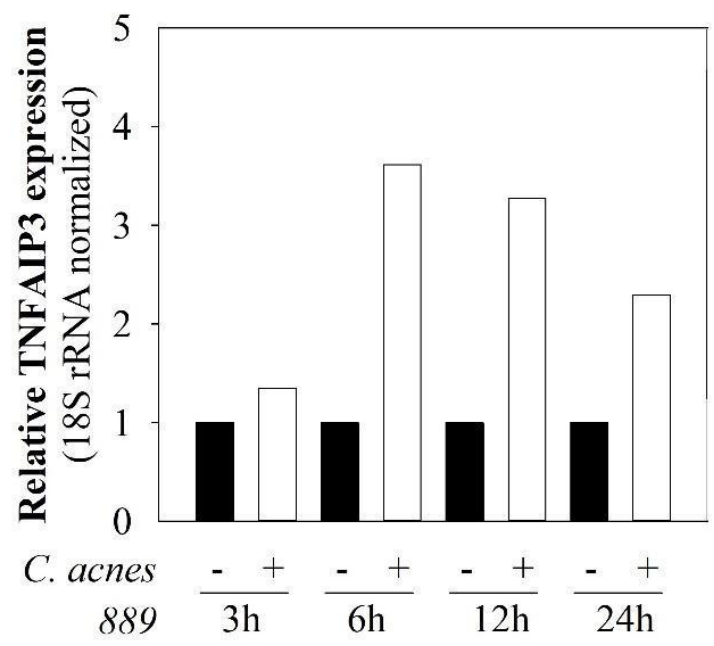

B

TNFAIP3

actin

C. acnes

\begin{tabular}{c}
$-25 \quad 50 \quad 100 \quad 200 \quad 300$ \\
\hline $24 \mathrm{~h}$
\end{tabular}

Supplementary figure 3. The effect of JNK and NF-KB inhibition on C. acnes-induced TNFAIP3 levels. HPV-KER cells were plated and pretreated with selective inhibitors for JNK, and NF- $\kappa \mathrm{B}$ or, as a control, DMSO for 1 hour. For mRNA expression studies $C$. acnes challenge was performed for 12 hours and analyzed by real-time RT-PCR. All data were normalized to the 18S rRNA and compared to DMSO-treated control samples. Statistical analyzes: paired, two-sample t-test with Holm-Bonferroni correction, $C$. acnes-induced TNFAIP3 expression were compared, \# $\mathrm{p}<0.05$. 


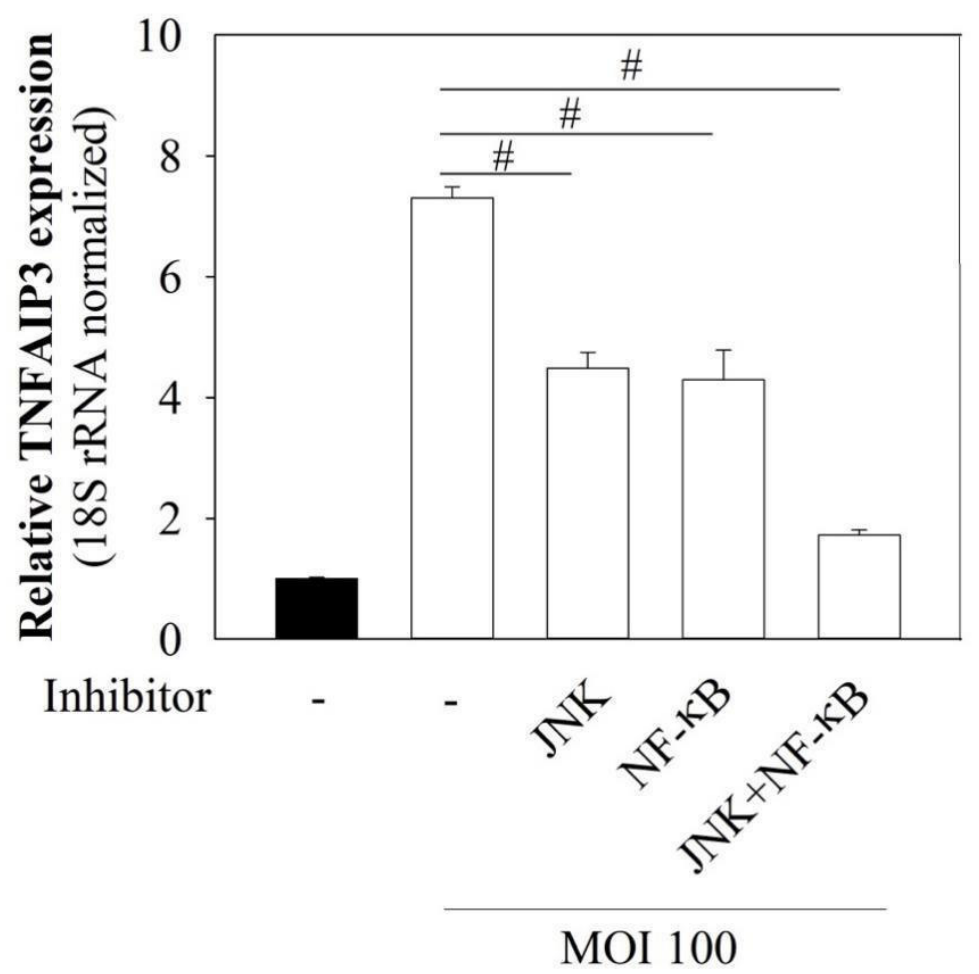

\section{REFERENCES}

1. Eyerich S, Eyerich K, Traidl-Hoffmann C, Biedermann T. Cutaneous Barriers and Skin Immunity: Differentiating A Connected Network. Trends Immunol 2018; 39: 315-327.

2. Gallo RL, Nakatsuji T. Microbial symbiosis with the innate immune defense system of the skin. J Invest Dermatol 2011; 131: 1974-1980.

3. de Koning HD, Rodijk-Olthuis D, van Vlijmen-Willems IM, Joosten LA, Netea MG, Schalkwijk J, et al. A comprehensive analysis of pattern recognition receptors in normal and inflamed human epidermis: upregulation of dectin-1 in psoriasis. J Invest Dermatol 2010; 130: 26112620.

4. Pivarcsi A, Kemeny L, Dobozy A. Innate immune functions of the keratinocytes. A review. Acta Microbiol Immunol Hung 2004; 51: 303-310.

5. Liew FY, Xu D, Brint EK, O'Neill LA. Negative regulation of toll-like receptor-mediated immune responses. Nat Rev Immunol 2005; 5: 446-458.

6. Oh J, Conlan S, Polley EC, Segre JA, Kong HH. Shifts in human skin and nares microbiota of healthy children and adults. Genome Med 2012; 4: 77.

7. Grice EA, Segre JA. The skin microbiome. Nat Rev Microbiol 2011; 9: 244-253.

8. Kim J, Ochoa MT, Krutzik SR, Takeuchi O, Uematsu S, Legaspi AJ, et al. Activation of toll-like receptor 2 in acne triggers inflammatory cytokine responses. J Immunol 2002; 169: 1535-1541.

9. Pivarcsi A, Bodai L, Rethi B, Kenderessy-Szabo A, Koreck A, Szell M, et al. Expression and function of Toll-like receptors 2 and 4 in human keratinocytes. Int Immunol 2003; 15: 721-730.

10. Nguyen CT, Sah SK, Zouboulis CC, Kim TY. Inhibitory effects of superoxide dismutase 3 on Propionibacterium acnes-induced skin inflammation. Sci Rep 2018; 8: 4024. 
11. Wang YY, Ryu AR, Jin S, Jeon YM, Lee MY. Chlorin e6-Mediated Photodynamic Therapy Suppresses P. acnes-Induced Inflammatory Response via NFkappaB and MAPKs Signaling Pathway. PLoS One 2017; 12: e0170599.

12. Pretsch A, Nagl M, Schwendinger K, Kreiseder B, Wiederstein M, Pretsch D, et al. Antimicrobial and anti-inflammatory activities of endophytic fungi Talaromyces wortmannii extracts against acne-inducing bacteria. PLoS One 2014; 9: e97929.

13. Simanski $M$, Erkens AS, Rademacher F, Harder J. Staphylococcus epidermidis-induced Interleukin-1 Beta and Human Beta-defensin-2 Expression in Human Keratinocytes is Regulated by the Host Molecule A20 (TNFAIP3). Acta Derm Venereol 2019; 99: 181-187.

14. Danis J, Janovak L, Guban B, Goblos A, Szabo K, Kemeny L, et al. Differential InflammatoryResponse Kinetics of Human Keratinocytes upon Cytosolic RNA- and DNA-Fragment Induction. Int J Mol Sci 2018; 19.

15. Megyeri K, Orosz L, Bolla S, Erdei L, Razga Z, Seprenyi G, et al. Propionibacterium acnes Induces Autophagy in Keratinocytes: Involvement of Multiple Mechanisms. J Invest Dermatol 2018; 138: $750-759$.

16. Tax G, Urban E, Palotas Z, Puskas R, Konya Z, Biro T, et al. Propionic Acid Produced by Propionibacterium acnes Strains Contri-butes to Their Pathogenicity. Acta Derm Venereol 2016; 96: 43-49.

17. Bolla BS, Erdei L, Urban E, Burian K, Kemeny L, Szabo K. Cutibacterium acnes regulates the epidermal barrier properties of HPV-KER human immortalized keratinocyte cultures. Sci Rep 2020; 10: 12815.

18. Trivedi NR, Gilliland KL, Zhao W, Liu W, Thiboutot DM. Gene array expression profiling in acne lesions reveals marked upregulation of genes involved in inflammation and matrix remodeling. J Invest Dermatol 2006; 126: 1071-1079.

19. Nagy I, Pivarcsi A, Koreck A, Szell M, Urban E, Kemeny L. Distinct strains of Propionibacterium acnes induce selective human beta-defensin-2 and interleukin-8 expression in human keratinocytes through toll-like receptors. J Invest Dermatol 2005; 124: 931-938.

20. Lheure C, Grange PA, Ollagnier G, Morand P, Desire N, Sayon S, et al. TLR-2 Recognizes Propionibacterium acnes CAMP Factor 1 from Highly Inflammatory Strains. PLoS One 2016; 11: e0167237.

21. Sohn KC, Back SJ, Choi DK, Shin JM, Kim SJ, Im M, et al. The inhibitory effect of A20 on the inflammatory reaction of epidermal keratinocytes. Int J Mol Med 2016; 37: 1099-1104.

22. Tiruppathi C, Soni D, Wang DM, Xue J, Singh V, Thippegowda PB, et al. The transcription factor DREAM represses the deubiquitinase A20 and mediates inflammation. Nat Immunol 2014; 15 : 239-247.

23. Lai TY, Wu SD, Tsai MH, Chuang EY, Chuang LL, Hsu LC, et al. Transcription of Tnfaip3 is regulated by NF-kappaB and p38 via C/EBPbeta in activated macrophages. PLoS One 2013; 8 : e73153.

24. Szabo K, Erdei L, Bolla BS, Tax G, Biro T, Kemeny L. Factors shaping the composition of the cutaneous microbiota. Br J Dermatol 2017; 176: 344-351.

25. Szegedi A, Dajnoki Z, Biro T, Kemeny L, Torocsik D. Acne: Transient Arrest in the Homeostatic Host-Microbiota Dialog? Trends Immunol 2019; 40: 873-876.

26. Kollisch G, Kalali BN, Voelcker V, Wallich R, Behrendt H, Ring J, et al. Various members of the Toll-like receptor family contribute to the innate immune response of human epidermal keratinocytes. Immunology 2005; 114: 531-541.

27. Steinhoff M, Brzoska T, Luger TA. Keratinocytes in epidermal immune responses. Curr Opin Allergy Clin Immunol 2001; 1: 469-476.

28. Sanford JA, Gallo RL. Functions of the skin microbiota in health and disease. Semin Immunol 2013; 25: 370-377.

29. Malynn BA, Ma A. A20: A multifunctional tool for regulating immunity and preventing disease. Cell Immunol 2019; 340: 103914. 
30. Das T, Chen Z, Hendriks RW, Kool M. A20/Tumor Necrosis Factor alpha-Induced Protein 3 in Immune Cells Controls Development of Autoinflammation and Autoimmunity: Lessons from Mouse Models. Front Immunol 2018; 9: 104.

31. Catrysse L, Vereecke L, Beyaert R, van Loo G. A20 in inflammation and autoimmunity. Trends Immunol 2014; 35: 22-31.

32. Stuart PE, Nair RP, Tsoi LC, Tejasvi T, Das S, Kang HM, et al. Genome-wide Association Analysis of Psoriatic Arthritis and Cutaneous Psoriasis Reveals Differences in Their Genetic Architecture. Am J Hum Genet 2015; 97: 816-836.

33. Dieguez-Gonzalez R, Calaza M, Perez-Pampin E, Balsa A, Blanco FJ, Canete JD, et al. Analysis of TNFAIP3, a feedback inhibitor of nuclear factor-kappaB and the neighbor intergenic $6 q 23$ region in rheumatoid arthritis susceptibility. Arthritis Res Ther 2009; 11: R42.

34. Kawasaki A, Ito S, Furukawa H, Hayashi T, Goto D, Matsumoto I, et al. Association of TNFAIP3 interacting protein 1, TNIP1 with systemic lupus erythematosus in a Japanese population: a case-control association study. Arthritis Res Ther 2010; 12: R174.

35. Perga S, Martire S, Montarolo F, Navone ND, Calvo A, Fuda G, et al. A20 in Multiple Sclerosis and Parkinson's Disease: Clue to a Common Dysregulation of Anti-Inflammatory Pathways? Neurotox Res 2017; 32: 1-7.

36. Celli A, Sanchez S, Behne M, Hazlett T, Gratton E, Mauro T. The epidermal $\mathrm{Ca}(2+)$ gradient: Measurement using the phasor representation of fluorescent lifetime imaging. Biophys J 2010; 98: 911-921.

37. Sours-Brothers $\mathrm{S}, \mathrm{Ma} \mathrm{R}$, Koulen $\mathrm{P}$. Ca2+-sensitive transcriptional regulation: direct DNA interaction by DREAM. Front Biosci (Landmark Ed) 2009; 14: 1851-1856.

38. Devos M, Mogilenko DA, Fleury S, Gilbert B, Becquart C, Quemener S, et al. Keratinocyte Expression of A20/TNFAIP3 Controls Skin Inflammation Associated with Atopic Dermatitis and Psoriasis. J Invest Dermatol 2019; 139: 135-145.

39. Fitz-Gibbon S, Tomida S, Chiu BH, Nguyen L, Du C, Liu M, et al. Propionibacterium acnes strain populations in the human skin microbiome associated with acne. J Invest Dermatol 2013; 133: 2152-2160.

40. Dagnelie MA, Corvec S, Saint-Jean M, Nguyen JM, Khammari A, Dreno B. Cutibacterium acnes phylotypes diversity loss: a trigger for skin inflammatory process. J Eur Acad Dermatol Venereol 2019; 33: 2340-2348.

41. Jasson F, Nagy I, Knol AC, Zuliani T, Khammari A, Dreno B. Different strains of Propionibacterium acnes modulate differently the cutaneous innate immunity. Exp Dermatol 2013; 22: 587-592.

42. Dreno B, Pecastaings $S$, Corvec $S$, Veraldi S, Khammari A, Roques C. Cutibacterium acnes (Propionibacterium acnes) and acne vulgaris: a brief look at the latest updates. J Eur Acad Dermatol Venereol 2018; 32 Suppl 2: 5-14.

43. Erdei L, Bolla BS, Bozo R, Tax G, Urban E, Kemeny L, et al. TNIP1 Regulates Cutibacterium acnesInduced Innate Immune Functions in Epidermal Keratinocytes. Front Immunol 2018; 9: 2155.

44. Hu J, Wang G, Liu X, Zhou L, Jiang M, Yang L. A20 is critical for the induction of Pam3CSK4tolerance in monocytic THP-1 cells. PLoS One 2014; 9: e87528.

45. Lippens S, Lefebvre S, Gilbert B, Sze M, Devos M, Verhelst K, et al. Keratinocyte-specific ablation of the NF-kappaB regulatory protein A20 (TNFAIP3) reveals a role in the control of epidermal homeostasis. Cell Death Differ 2011; 18: 1845-1853.

46. Dreno B. Bacteriological resistance in acne: A call to action. Eur J Dermatol 2016; 26: 127-132.

47. Orfanos CE, Zouboulis CC. Oral retinoids in the treatment of seborrhoea and acne. Dermatology 1998; 196: 140-147. 